\title{
A Deep Chandra Survey of the Globular Cluster 47 Tucanae: Catalog of Point Sources
}

\section{Citation}

Heinke, C. O., J. E. Grindlay, P. D. Edmonds, H. N. Cohn, P. M. Lugger, F. Camilo, S. Bogdanov, and P. C. Freire. 2005. "A Deep Chandra Survey of the Globular Cluster 47 Tucanae: Catalog of Point Sources." The Astrophysical Journal 625 (2): 796-824. https://doi.org/10.1086/429899.

\section{Permanent link}

http://nrs.harvard.edu/urn-3:HUL.InstRepos:41399912

\section{Terms of Use}

This article was downloaded from Harvard University's DASH repository, and is made available under the terms and conditions applicable to Other Posted Material, as set forth at http:// nrs.harvard.edu/urn-3:HUL.InstRepos:dash.current.terms-of-use\#LAA

\section{Share Your Story}

The Harvard community has made this article openly available.

Please share how this access benefits you. Submit a story.

Accessibility 


\title{
A DEEP CHANDRA SURVEY OF THE GLOBULAR CLUSTER 47 TUCANAE: CATALOG OF POINT SOURCES
}

\author{
C. O. Heinke, ${ }^{1,2,3}$ J. E. Grindlay, ${ }^{1}$ P. D. Edmonds, ${ }^{1}$ H. N. Cohn, ${ }^{4}$ P. M. Lugger,${ }^{4}$ F. Camilo, ${ }^{5}$ \\ S. Bogdanov, ${ }^{1}$ and P. C. Freire ${ }^{6}$ \\ Received 2004 September 9; accepted 2005 February 18
}

\begin{abstract}
We have detected $300 \mathrm{X}$-ray sources within the half-mass radius (2.'79) of the globular cluster 47 Tucanae in a deep (281 ks) Chandra exposure. We perform photometry and simple spectral fitting for our detected sources and construct luminosity functions, X-ray color-magnitude, and color-color diagrams. Eighty-seven X-ray sources show variability on timescales from hours to years. Thirty-one of the new X-ray sources are identified with chromospherically active binaries from the catalogs of Albrow and coworkers. The radial distributions of detected sources imply that roughly 70 are background sources of some kind. The radial distribution of the known millisecond pulsar (MSP) systems is consistent with that expected from mass segregation, if the average neutron star mass is $1.39 \pm 0.19 M_{\odot}$. Most source spectra are well fitted by thermal plasma models, except for quiescent lowmass X-ray binaries (qLMXBs; containing accreting neutron stars) and MSPs. We identify three new candidate qLMXBs with relatively low X-ray luminosities. One of the brightest cataclysmic variables (CVs; X10) shows evidence (a $4.7 \mathrm{hr}$ period pulsation and strong soft X-ray emission) for a magnetically dominated accretion flow as in AM Her systems. Most of the bright CVs require intrinsic $N_{\mathrm{H}}$ columns of order $10^{21} \mathrm{~cm}^{-2}$, suggesting a possible DQ Her nature. A group of X-ray sources associated with bright (sub)giant stars also requires intrinsic absorption. By comparing the X-ray colors, luminosities, variability, and quality of spectral fits of the detected MSPs to those of unidentified sources, we estimate that a total of $\sim 25$ MSPs exist in 47 Tuc ( $<60$ at $95 \%$ confidence), regardless of their radio beaming fraction. We estimate that the total number of neutron stars in 47 Tuc is of order 300 , reducing the discrepancy between theoretical neutron star retention rates and observed neutron star populations in globular clusters. Comprehensive tables of source properties and simple spectral fits are provided electronically.
\end{abstract}

Subject headings: globular clusters: individual (NGC 104) — novae, cataclysmic variables —

pulsars: general - stars: neutron - X-rays: binaries

Online material: color figures, machine-readable tables

\section{INTRODUCTION}

Globular cluster cores have significantly enhanced dynamical interactions that produce a variety of close binary systems, such as blue straggler stars and millisecond radio pulsars (Clark 1975; Hut et al. 1992; Bailyn \& Pinsonneault 1995; Johnston et al. 1992). X-ray studies, especially with the Chandra X-Ray Observatory, have been extremely effective in discovering compact binaries in globular clusters (for a review see Verbunt \& Lewin 2004). The brightest $X$-ray sources $(0.5-2.5 \mathrm{keV} X$-ray luminosity $L_{\mathrm{X}}=10^{35.5}-10^{37} \mathrm{ergs} \mathrm{s}^{-1}$ ) seen in a few (12) globular clusters are persistent or transient low-mass X-ray binaries (LMXBs) containing neutron stars (Grindlay et al. 1984; Lewin \& Joss 1983; Sidoli et al. 2001). The lower luminosity X-ray sources $\left(L_{\mathrm{X}}=10^{29}-10^{34} \mathrm{ergs} \mathrm{s}^{-1}\right)$ include several kinds of systems with different $\mathrm{X}$-ray emission mechanisms. These include accreting cataclysmic variables (CVs; Hertz \& Grindlay 1983; Cool et al. 1995), quiescent low-mass X-ray binaries

\footnotetext{
${ }^{1}$ Harvard-Smithsonian Center for Astrophysics, 60 Garden Street, Cambridge, MA 02138; cheinke@cfa.harvard.edu,josh@cfa.harvard.edu,pedmonds@cfa .harvard.edu,sbogdanov@cfa.harvard.edu.

2 Department of Physics and Astronomy, Northwestern University, 2145 Sheridan Road, Evanston, IL 60208.

${ }^{3}$ Lindheimer Postdoctoral Fellow.

4 Department of Astronomy, Indiana University, Swain West 319, Bloomington, IN 47405; cohn@indiana.edu,lugger@indiana.edu.

5 Columbia Astrophysics Laboratory, Columbia University, 550 West 120th Street, New York, NY 10027; fernando@astro.columbia.edu.

6 National Astronomy and Ionosphere Center, Arecibo Observatory, HC03 Box 53995, Arecibo, PR 00612; pfreire@naic.edu.
}

(qLMXBs) where accretion onto a neutron star is stopped or greatly reduced (Hertz \& Grindlay 1983; Verbunt et al. 1984; Campana et al. 1998), millisecond radio pulsars (MSPs), thought to be descendants of LMXBs (Saito et al. 1997; Grindlay et al. 2002), and X-ray active binaries (ABs) consisting of normal stars in tidally locked short-period binaries, where the fast rotation rate drives increased coronal activity (Bailyn et al. 1990; Dempsey et al. 1993; Grindlay et al. 2001a, hereafter G01a).

The globular cluster 47 Tuc is massive $\left(M \sim 10^{6} M_{\odot}\right.$, Pryor \& Meylan 1993) and of relatively high stellar concentration, although not core collapsed (Harris 1996). In this paper we use a distance of $4.85 \mathrm{kpc}$ (Gratton et al. 2003), a neutral gas column of $N_{\mathrm{H}}=1.3 \times 10^{20} \mathrm{~cm}^{-2}$ [from $E(B-V)$ of 0.024 and $N_{\mathrm{H}} / E(B-V)=5.5 \times 10^{21} \mathrm{~cm}^{-2}$; Gratton et al. 2003; Predehl \& Schmitt 1995; Cardelli et al. 1989], and a core radius of 24" (Howell et al. 2000). It is one of the clusters with the highest predicted close encounter frequencies, implying a substantial population of binaries whose properties have been altered by close encounters with other stars or binaries (Verbunt \& Hut 1987; Pooley et al. 2003). It has a bimodal distribution of blue straggler stars (Bailyn \& Pinsonneault 1995), with a high density in the core of presumably collisional blue stragglers (Ferraro et al. 2001) and another population of probable primordial blue stragglers in the halo (Ferraro et al. 2004). Various attempts to derive the fraction of stars in binaries in 47 Tuc have given results from 10\%-18\% (Albrow et al. 2001, hereafter A01) to $\$ 5 \%$ (Anderson 1997; Ivanova et al. 2005a). Measuring the various binary populations of 47 Tuc is critical to understanding and modeling the dynamical encounters between 
binaries that lead to the production of X-ray sources in globular clusters.

The globular cluster 47 Tuc is well known for its large number of MSPs, which are believed to have been produced by the evolution of LMXBs that were themselves produced through interactions of binaries and neutron stars (Bhattacharya \& van den Heuvel 1991; Hut et al. 1992; Rasio et al. 2000). A total of 22 MSPs have been discovered in 47 Tuc to date (Manchester et al. 1991; Camilo et al. 2000; X and Y still unpublished), of which 17 have known radio timing positions (Freire et al. 2001, 2003; 47 Tuc R's timing solution is not yet published; P. C. Freire et al. 2005, in preparation), and an additional MSP (47 Tuc W) has a position determined by matching a known MSP orbital period to the period of a variable optical star and X-ray source (Edmonds et al. 2002a). Thirteen are members of binary systems, and at least two (possibly up to four) MSP companions have been detected using the Hubble Space Telescope (HST; Edmonds et al. 2001, 2002a, 2003a). Although most of the MSPs in 47 Tuc have flux densities below the sensitivity of the Parkes radio telescope used to detect them, strong interstellar scintillation effects occasionally bring their signals up to detectability. Assuming that the luminosity distribution of 47 Tuc pulsars is similar to that of field systems, Camilo et al. (2000) estimated a total MSP population of $\sim 200$ systems. Grindlay et al. (2002) detected blackbody-like thermal emission from the surface of most of the MSPs in 47 Tuc, presumably hot polar caps. They estimated a total MSP population in 47 Tuc of 35-90 based on the X-ray properties of the X-raydetected MSPs compared to other 47 Tuc X-ray sources. Edmonds et al. (2003b) provided evidence that the total 47 Tuc MSP population was closer to 30-40, from optical identifications of X-ray sources. McConnell et al. (2004) inferred that the observable radio MSP population in 47 Tuc is less than 30, using observations that attempted to detect the integrated radio flux from a population of unresolved pulsars. Since MSPs are highly compact, X-rays from surface hot spots will be bent by gravity to allow observers to see $\sim 75 \%$ of the neutron star surface (Pechenick et al. 1983; Beloborodov 2002; S. Bogdanov et al. 2005 , in preparation). This virtually ensures that MSPs whose radio beams do not intercept the Earth should still be detectable in X-rays and, with the lack of scintillation and eclipses due to ionized gas (as seen in the radio; Camilo et al. 2000), allows more complete study of MSPs by X-ray observations than radio and places constraints on the radio beaming fraction (Grindlay et al. 2002).

Low-luminosity X-ray emission from 47 Tuc $\left(L_{\mathrm{X}} \sim 10^{34} \mathrm{ergs}\right.$ $\mathrm{s}^{-1}$ ) was first identified with the Einstein Observatory and suggested to be due to CVs (Hertz \& Grindlay 1983). Deep pointings by the ROSAT satellite measured a lower X-ray luminosity and resolved the emission into nine sources, of which several showed variability (Hasinger et al. 1994; Verbunt \& Hasinger 1998). The first Chandra (ACIS-I) image of the cluster resolved 104 sources within a $2^{\prime}$ by 2 '. 5 region including the core (G01a). A later study of the same data extending to a radius of $4^{\prime}$ studied the X-ray emission from most of the MSPs with known positions and measured the radial distribution of the X-ray sources (Grindlay et al. 2002). X-ray spectra of the two brightest X-ray sources were well fitted by hydrogen atmosphere models for neutron stars, consistent with the spectra of known qLMXBs (Heinke et al. 2003b).

Optical identification efforts began shortly after the discovery of X-ray emission from 47 Tuc but have proven difficult owing to the intense crowding at the center of 47 Tuc and the faintness of optical counterparts. Auriere et al. (1989) suggested a possible CV counterpart, AKO 9, to the Einstein X-ray source. Paresce et al. (1992), Paresce \& de Marchi (1994), and Shara et al. (1996) identified three CVs in 47 Tuc through HST imaging, which were later identified with ROSAT X-ray sources (Verbunt \& Hasinger 1998; G01a). Definitive identifications became possible using Chandra positions and HST data (especially the exceptional GO-8267 8.3 day time series; Gilliland et al. 2000) to search for variability and unusual colors (G01a; Edmonds et al. 2001, 2002a, 2002b, 2003a, 2003b). These searches at X-ray positions produced secure optical identifications of $22 \mathrm{CVs}$, $29 \mathrm{ABs}$, one eclipsing qLMXB, and two MSP companions (two additional possible MSP companions were also identified).

Chandra X-ray studies of numerous other globular clusters have given insight into the nature and formation processes of the brighter $\left(L_{\mathrm{X}} \gtrsim 10^{31} \mathrm{ergs} \mathrm{s}^{-1}\right)$ sources (Pooley et al. $2002 \mathrm{a}$, 2003; Heinke et al. 2003c). 47 Tuc is an ideal target for study of the fainter X-ray sources, including most MSPs and ABs. This is due to its high rate of close encounters (a product of its large mass and high density), relatively small distance, low reddening, reasonably large angular core size (reducing X-ray crowding), and excellent multiwavelength coverage (also including far-UV HST spectra and imaging; Knigge et al. 2002).

In this paper we describe the Chandra X-ray data and attempt to understand the nature and characteristics of the faint X-ray sources. We focus on the 2002 data but include some analysis of the original 2000 Chandra data set. Additional papers will focus on particular aspects of the X-ray data, individual sources, and analysis of archival and new simultaneous $H S T$ data. In $\S 2$ we describe the observations and initial analysis, including source detection, optical counterpart identifications, and extraction of $\mathrm{X}$-ray data. In $\S 3$ we describe searches for short- and long-term variability. In $\S 4$ we discuss the radial distributions of our sources, and in $\S 5$ we utilize the X-ray "color" information. In $\S 6$ we discuss the luminosity functions of the various source classes. In $\S 7$ we perform simple spectral fitting and briefly discuss some unusual objects we identify. In $\S 8$ we discuss the meaning of our results and constrain the populations of unidentified sources.

\section{OBSERVATIONS AND ANALYSIS}

The data used in this paper are from the 2000 and 2002 Chandra observations of the globular cluster 47 Tuc. The 2000 observations, initially described in G01a, were performed with the ACIS-I CCD array at the telescope focus, while the 2002 observations placed the back-illuminated ACIS-S aim point at the focus for maximum low-energy sensitivity. Five consecutive observations were performed in 2000, as listed in Table 1, with three short observations interleaved to obtain spectra of bright sources with little pileup. The 2002 observations (four pairs of $\sim 65 \mathrm{ks}$ full-frame exposures followed by $\sim 5 \mathrm{ks} 1 / 4$ subarray exposures; see Table 1) were designed to probe variability on a range of timescales from hours to weeks. This was intended to allow distinctions between relatively constant thermal MSP emission and flaring behavior from ABs. The first two 2002 exposure pairs were run consecutively for a total of 1.7 days, with the remaining pairs following at intervals of 1.4 and 7.4 days.

Our data analysis for all observations begins with the Level 1 processed event lists provided by the Chandra X-Ray Center pipeline processing. We used the CIAO data analysis tools ${ }^{7}$ for initial data processing. We reprocessed the 2000 observations using the CTI correction algorithm implemented in CIAO 3.02

\footnotetext{
7 Ver. 3.02, http://cxc.harvard.edu/ciao.
} 
TABLE 1

Summary of Chandra Observations

\begin{tabular}{|c|c|c|c|c|c|}
\hline Sequence, ObsID & Start Time & $\begin{array}{l}\text { Exposure } \\
\text { (s) }\end{array}$ & Aim Point & $\begin{array}{l}\text { Frame Time } \\
\text { (s) }\end{array}$ & CCDs \\
\hline 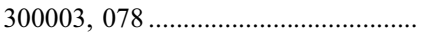 & 2000 Mar 16 07:18:30 & 3875 & ACIS-I & 0.94 & $1 / 4$ \\
\hline $300028,953 \ldots \ldots$. & 2000 Mar 16 08:39:44 & 31421 & ACIS-I & 3.24 & 6 \\
\hline $300029,954 \ldots \ldots$. & 2000 Mar 16 18:03:03 & 845 & ACIS-I & 0.54 & $1 / 8$ \\
\hline $300030,955 \ldots \ldots \ldots$ & 2000 Mar 16 18:33:03 & 31354 & ACIS-I & 3.24 & 6 \\
\hline 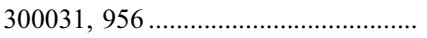 & 2000 Mar 17 03:56:23 & 4656 & ACIS-I & 0.94 & $1 / 4$ \\
\hline $400215,2735 \ldots$. & 2002 Sep 29 16:59:00 & 65237 & ACIS-S & 3.14 & 5 \\
\hline 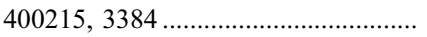 & 2002 Sep 30 11:38:22 & 5307 & ACIS-S & 0.84 & $1 / 4$ \\
\hline 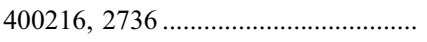 & 2002 Sep 30 13:25:32 & 65243 & ACIS-S & 3.14 & 5 \\
\hline 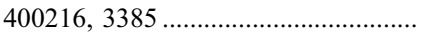 & 2002 Oct $0108: 13: 32$ & 5307 & ACIS-S & 0.84 & $1 / 4$ \\
\hline 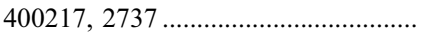 & 2002 Oct 02 18:51:10 & 65243 & ACIS-S & 3.14 & 5 \\
\hline 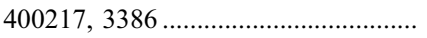 & 2002 Oct 03 13:38:21 & 5545 & ACIS-S & 0.84 & $1 / 4$ \\
\hline 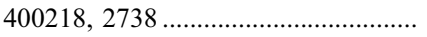 & 2002 Oct 11 01:42:59 & 68771 & ACIS-S & 3.14 & 5 \\
\hline 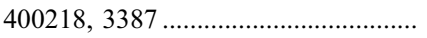 & 2002 Oct $1121: 23: 12$ & 5735 & ACIS-S & 0.84 & $1 / 4$ \\
\hline
\end{tabular}

NoTE.- Subarrays are indicated by fractional numbers of CCDs.

acis_process_events. Both the 2000 and 2002 observations were reprocessed to remove the 0 ". 25 pixel randomization added in standard processing. We removed bad columns, bad pixels, and events not exhibiting one of the "standard ASCA grades" $(0,2,3,4,6)$ and applied the good time intervals produced by the $\mathrm{CXC}$ pipeline. We also applied the new time-dependent gain correction software (A. Vikhlinin 2003, private communication ${ }^{8}$ ) to all event files.

We produced two sets of event lists for each observation for different analysis purposes: an imaging event list for source detection and positioning, and a spectral event list for use in extracting spectra and light curves. For imaging, we removed times of high background more aggressively and removed events identified with the CIAO procedure acis_detect_afterglow. Cosmic rays striking the detector release an initial charge that is generally rejected, but they often release residual charge over 10-30 s. The acis_detect_afterglow program identifies likely cosmic-ray afterglows, which can otherwise be mistaken for true sources by detection algorithms. However, the procedure also flags counts from real sources (Feigelson et al. 2002), ${ }^{9}$ so for extraction of spectra and light curves we use event lists with flagged events included.

Inspection of the background light curve shows one period of significant flaring, $7.1 \mathrm{ks}$ at the end of ObsID 2736, increasing through ObsID 3385. A milder increase in background is also seen for $13 \mathrm{ks}$ of ObsID 2738 and ObsID 3387. We include all of ObsIDs 2738 and 3387 in our analyses, since this gives us a higher signal-to-noise ratio for our detected sources. For imaging analysis (where our goal is the detection of faint sources), we excise the period of high background from ObsID 2736 and do not use ObsID 3385 (leaving an effective exposure time of 273,964 s, total time). For spectral and light-curve extraction, we use all of ObsID 2736 but omit ObsID 3385 (leaving good time of $281,074 \mathrm{~s}$ for sources within the area covered by the subarrays, 275,529 s for those outside). Since the total background within a typical $\left(5 \operatorname{arcsec}^{2}\right)$ source extraction area is less than 4 photons, more aggressive background flaring removal does not improve our signal-to-noise ratio for sources with more than 20 counts. For the 2000 observations, the total good time is $72,155 \mathrm{~s}$

For this paper we confine ourselves to analysis of sources within the half-mass radius of 47 Tuc, 2.79 (Harris 1996). We

\footnotetext{
${ }^{8}$ See http://cxc.harvard.edu/contrib/alexey/tgain/tgain.html.

9 See also http://asc.harvard.edu/ciao/threads/acisdetectafterglow.
}

choose this radius for easy comparison with analyses of other clusters (e.g., Pooley et al. 2002a, 2002b, 2003; Heinke et al. 2003a; Bassa et al. 2004) and because it strikes a balance between inclusion of almost all real cluster sources and exclusion of most foreground and background sources at the flux levels we are most interested in. X-ray sources or MSPs associated with the cluster beyond the half-mass radius are known in corecollapsed clusters like NGC 6752 (D'Amico et al. 2002) and M15 (Phinney \& Sigurdsson 1991), as well as in $\omega$ Cen (Cool et al. 2002; Gendre et al. 2003), which has not yet dynamically relaxed. However, in relaxed King model clusters the relatively massive binaries are expected to be more centrally concentrated (Verbunt \& Meylan 1988; Fregeau et al. 2003). The radial distribution studies of the 2000 Chandra data by Grindlay et al. (2002) and Edmonds et al. (2003b) showed that all identified $\mathrm{X}$-ray source populations in 47 Tuc are highly concentrated in the core, as expected since both binaries and single MSPs are significantly more massive than typical stars in 47 Tuc.

\subsection{Source Detection}

We performed all data reduction separately for the 2000 and 2002 observations, since they were taken with different detectors. The CIAO tool wavdetect was used to measure the positions of the three brightest sources in different exposures. We applied small shifts to align the later 2002 exposures with the first 2002 exposure $\left(+0.028,+0\right.$ " $^{\prime \prime} 023 ;-0 .{ }^{\prime \prime} 012,+0.063 ;-0{ }^{\prime \prime} 026$, +0 ". 041 added to the right ascension and declination of the three later exposures, with rms residuals of 0 ". 013,0 , 014 remaining). We reprojected the exposures in each group to match the alignment of the first long exposure in each year and merged each group of observations.

For the 2000 observations we performed wavdetect runs at full resolution in the $0.5-2$ and $0.5-6 \mathrm{keV}$ energy bands. For the 2002 observations we used bands of $0.3-2$ and $0.3-6 \mathrm{keV}$. For each wavdetect run we selected scales of 1.0, 1.414, 2.0, and 2.828 pixels, for optimal detection and separation of point sources near on-axis. We selected a threshold probability of $1 \times 10^{-5}$, designed to give one false source per $10^{5}$ pixels. Each wavdetect run (assuming that the trials and pixels are independent) should thus give of order 3.6 spurious sources, given the 0.492 pixel size and 2.79 radius searched. (Our results tend to indicate that this is a slight overestimate; see below.) Harder energy bands failed to reveal more than two or three marginal additional sources not detected in the other bands. Inspection of 
TABLE 2

47 Tuc Basic X-Ray Source Properties, 2002 Data Set

\begin{tabular}{|c|c|c|c|c|c|c|c|c|c|c|c|c|}
\hline \multicolumn{2}{|r|}{ NAMES } & \multicolumn{2}{|c|}{ Position } & \multirow[b]{2}{*}{$\begin{array}{c}\text { Distance } \\
(\operatorname{arcsec}) \\
(5)\end{array}$} & \multicolumn{3}{|c|}{ Counts } & \multicolumn{2}{|c|}{$L_{\mathrm{X}}$} & \multirow[b]{2}{*}{$\begin{array}{l}\text { ID TYPE } \\
\text { (11) }\end{array}$} & \multirow[b]{2}{*}{$\begin{array}{c}\text { Other NAMES } \\
\text { (12) }\end{array}$} & \multirow[b]{2}{*}{$\begin{array}{l}\text { Notes } \\
\text { (13) }\end{array}$} \\
\hline $\begin{array}{l}\mathrm{W} \\
(1)\end{array}$ & $\begin{array}{l}\text { CXOGlb J } \\
\text { (2) }\end{array}$ & $\begin{array}{c}\alpha \\
(\mathrm{J} 2000.0) \\
(3)\end{array}$ & $\begin{array}{c}\delta \\
(\mathrm{J} 2000.0) \\
(4)\end{array}$ & & $\begin{array}{l}0.5-4.5 \\
\mathrm{keV} \\
(6)\end{array}$ & $\begin{array}{l}0.5-1.5 \\
\mathrm{keV} \\
(7)\end{array}$ & $\begin{array}{c}1.5-6 \\
\mathrm{keV} \\
(8)\end{array}$ & $\begin{array}{c}0.5-6.0 \\
\left(10^{30} \mathrm{ergs} \mathrm{s}^{-1}\right) \\
(9)\end{array}$ & $\begin{array}{c}0.5-2.5 \\
\left(10^{30} \mathrm{ergs} \mathrm{s}^{-1}\right) \\
(10)\end{array}$ & & & \\
\hline $46 \ldots \ldots \ldots \ldots \ldots$ & $002403.4-720451$ & 002403.4940 .001 & -720451.940 .01 & 8 & $31407.2_{-177.2}^{+179.1}$ & $26426.7_{-162.6}^{+164.0}$ & $4987.6_{-70.6}^{+72.1}$ & $1760.1_{-9.9}^{+10.0}$ & $1227.1_{-7.0}^{+7.0}$ & $\mathrm{qLX}$ & $\mathrm{X} 7$ & \\
\hline $42 \ldots \ldots \ldots \ldots \ldots$ & $002404.2-720458$ & 002404.2490 .001 & -720458.020 .01 & 7 & $16661.4_{-129.1}^{+111.0}$ & $11569.7_{-107.6}^{+1090.0}$ & $5954.6_{-77.2}^{+78.9}$ & $1107.5_{-8.4}^{+8.5}$ & $588.2_{-4.9}^{+4.9}$ & $\mathrm{CV}$ & X9 & DHY \\
\hline $58 \ldots \ldots \ldots \ldots \ldots$ & $002400.9-720453$ & 002400.9470 .001 & -720453.200 .01 & 20 & $11130.1_{-1055}^{+107.4}$ & $8723.8_{-99.4}^{+94.8}$ & $2422.4_{-49.2}^{+50.7}$ & $581.7_{-55}^{+5.6}$ & $429.2_{-4.1}^{+4.2}$ & $q L X$ & $\mathrm{X} 5$ & DHY \\
\hline $27 \ldots \ldots \ldots \ldots$ & $002406.3-720443$ & 002406.3740 .001 & -720443.000 .01 & 10 & $3947.1_{-62.8}^{+66.3}$ & $3241.3_{-56.9}^{+58.4}$ & $815.1_{-28.5}^{+30.3}$ & $259.3_{-4.1}^{+4.2}$ & $147.8_{-2.4}^{+2.5}$ & $\mathrm{CV}$ & $\mathrm{X} 10$ & DH \\
\hline $47 \ldots \ldots \ldots \ldots$ & $002403.4-720505$ & 002403.4500 .002 & $\begin{array}{llll}-72 & 05 & 05.28 & 0.01\end{array}$ & 15 & $2629.8_{-51.3}^{+53.3}$ & $1375.2_{-37.1}^{+36.9}$ & $1387.7_{-37.2}^{+38.9}$ & $147.6_{-2.8}^{+2.9}$ & $85.5_{-1.9}^{+1.4}$ & $\mathrm{AB}$ & $\ldots$ & DHY \\
\hline $56 \ldots \ldots \ldots \ldots$ & $002402.1-720542$ & 002402.1190 .002 & $\begin{array}{llll}-72 & 05 & 42.03 & 0.01\end{array}$ & 51 & $2590.4_{-50.9}^{+52.9}$ & $1487.5_{-38.6}^{+40.1}$ & $1222.9_{-35.0}^{+36.2}$ & $143.5_{-2.8}^{+2.8}$ & $86.2_{-1.9}^{+1.9}$ & $\mathrm{CV}$ & X6 & DHY \\
\hline $1 \ldots \ldots \ldots \ldots \ldots \ldots$ & $002416.9-720427$ & 002416.9680 .002 & $\begin{array}{llll}-72 & 04 & 27.21 & 0.01\end{array}$ & 59 & $1810.6_{-42.5}^{+44.5}$ & $1080.1_{-32.9}^{+34.3}$ & $804.7_{-28.3}^{+30.1}$ & $97.3_{-2.2}^{+2.8}$ & $59.7_{-1.5}^{+1.6}$ & $\mathrm{CV}$ & $\ldots$ & $\mathrm{H} ? \mathrm{Y}$ \\
\hline $2 \ldots \ldots \ldots \ldots \ldots$ & $002415.8-720436$ & 002415.8800 .002 & -720436.380 .01 & 51 & $1714.0_{-41.4}^{+43.4}$ & $846.7_{-29.1}^{+30.5}$ & $956.7_{-30.9}^{+32.6}$ & $96.1_{-2.3}^{+2.4}$ & $55.8_{-1.5}^{+1.5}$ & $\mathrm{CV}$ & $\mathrm{X} 13$ & DHY \\
\hline $51 \ldots \ldots \ldots \ldots \ldots$ & $002402.8-720449$ & 002402.8240 .003 & -720449.010 .01 & 11 & $1395.1_{-37.3}^{+39.3}$ & $891.1_{-29.8}^{+31.3}$ & $548.3_{-23.4}^{+25.1}$ & $82.9_{-2.2}^{+2.3}$ & $51.1_{-1.5}^{+1.5}$ & $\mathrm{CV}$ & $\ldots$ & DHY \\
\hline $45 \ldots \ldots \ldots \ldots$ & $002403.7-720423$ & 002403.7620 .003 & $\begin{array}{llll}-72 & 04 & 22.98 & 0.01\end{array}$ & 30 & $1320.5_{-36.3}^{+38.3}$ & $817.0_{-28.6}^{+30.8}$ & $545.4_{-23.3}^{+25.4}$ & $72.8_{-2.0}^{+2.1}$ & $46.2_{-1.4}^{+1.4}$ & $\mathrm{CV}$ & $\ldots$ & DH \\
\hline $125 \ldots \ldots \ldots \ldots$ & $002353.9-720350$ & 002353.9820 .003 & -720350.070 .01 & 81 & $1118.8_{-33.4}^{+35.4}$ & $929.1_{-30.5}^{+30.9}$ & $200.3_{-14.1}^{+15.9}$ & $67.8_{-2.0}^{+2.1}$ & $42.2_{-1.3}^{+1.3}$ & qLX? & $\mathrm{X} 4$ & $\mathrm{D} ? \mathrm{H}$ \\
\hline $37 \ldots \ldots \ldots \ldots$ & $002404.9-720451$ & 002404.9630 .003 & -720451.330 .01 & 1 & $1155.2_{-34.0}^{+35.8}$ & $1003.3_{-31.7}^{+33.1}$ & $154.6_{-12.4}^{+14.1}$ & $67.1_{-2.0}^{+2.1}$ & $47.1_{-1.4}^{+1.4}$ & qLX? & & DHY \\
\hline $25 \ldots \ldots \ldots \ldots$ & $002407.1-720545$ & 002407.1370 .003 & -720545.790 .01 & 54 & $1024.3_{-32.0}^{+34.0}$ & $646.9_{-25.4}^{+26.8}$ & $418.8_{-20.4}^{+22.4}$ & $56.8_{-1.7}^{+1.9}$ & $35.8_{-1.2}^{+1.2}$ & $\mathrm{CV}$ & $\mathrm{X} 11$ & $\mathrm{D}$ \\
\hline $30 \ldots \ldots \ldots \ldots$ & $002406.0-720456$ & 002405.9960 .003 & -720456.180 .01 & 5 & $875.9_{-29.6}^{+31.0}$ & $562.8_{-23.7}^{+25.4}$ & $347.3_{-18.6}^{+20.4}$ & $51.0_{-1.7}^{+1.8}$ & $31.6_{-1.1}^{+1.2}$ & $\mathrm{CV}$ & $\mathrm{X} 19$ & DY \\
\hline $17 \ldots \ldots \ldots \ldots$ & $002408.3-720431$ & 002408.3050 .003 & -720431.430 .01 & 25 & $756.9_{-27.5}^{+29.5}$ & $604.7_{-24.6}^{+26.0}$ & $159.7_{-12.6}^{-18.3}$ & $48.4_{-1.7}^{+1.9}$ & $29.9_{-1.1}^{+1.2}$ & qLX? & $\ldots$ & \\
\hline $64 \ldots \ldots \ldots \ldots . . . .$. & $002357.6-720502$ & 002357.6710 .003 & $\begin{array}{llll}-72 & 05 & 01.98 & 0.02\end{array}$ & 36 & $780.5_{-27.9}^{+30.3}$ & $492.0_{-22.2}^{+23.6}$ & $337.3_{-18.3}^{+20.0}$ & $46.4_{-1.6}^{+1.7}$ & $26.2_{-1.0}^{+1.1}$ & $\mathrm{AB}$ & $\ldots$ & DH? \\
\hline $23 \ldots \ldots \ldots \ldots$ & $002407.8-720441$ & 002407.8060 .004 & -720441.560 .02 & 15 & $712.3_{-26.7}^{+28.9}$ & $428.4_{-20.7}^{+22.1}$ & $293.5_{-17.1}^{+18.3}$ & $41.8_{-1.6}^{+1.7}$ & $28.6_{-1.1}^{+1.2}$ & $\mathrm{AB}$ & $\ldots$ & $\mathrm{HY}$ \\
\hline $53 \ldots \ldots \ldots \ldots \ldots$ & $002402.5-720511$ & 002402.5300 .003 & $\begin{array}{llll}-72 & 05 & 11.25 & 0.02\end{array}$ & 22 & $651.7_{-25.5}^{+27.6}$ & $368.9_{-19.2}^{+20.6}$ & $307.9_{-17.5}^{+19.1}$ & $36.8_{-1.4}^{+1.5}$ & $21.5_{-0.9}^{+1.0}$ & $\mathrm{CV}$ & $\ldots$ & $\mathrm{Y}$ \\
\hline $8 \ldots \ldots \ldots \ldots \ldots \ldots$ & $002410.7-720425$ & $0024 \quad 10.7470 .004$ & -720425.670 .02 & 36 & $589.5_{-24.2}^{+26.2}$ & $142.6_{-11.9}^{+13.3}$ & $529.5_{-23.0}^{+24.7}$ & $37.7_{-1.5}^{+1.6}$ & $14.0_{-0.7}^{+0.8}$ & $\mathrm{CV}$ & $\ldots$ & $\mathrm{Y}$ \\
\hline $36 \ldots \ldots \ldots \ldots$ & $002404.9-720455$ & 002404.9130 .003 & -720455.360 .02 & 3 & $491.1_{-22.1}^{+24.0}$ & $106.9_{-10.3}^{+11.8}$ & $509.8_{-22.6}^{+24.0}$ & $50.0_{-2.0}^{+2.2}$ & $9.4_{-0.7}^{+0.7}$ & $\mathrm{CV}$ & AKO 9 & $\mathrm{D} ? \mathrm{H}$ ? \\
\hline $32 \ldots \ldots \ldots \ldots \ldots$ & $002405.6-720449$ & 002405.6450 .003 & -720449.200 .02 & 3 & $544.6_{-23.3}^{+25.3}$ & $400.0_{-200}^{+21.4}$ & $158.0_{-125}^{+14.3}$ & $37.5_{-16}^{+1.7}$ & $22.5_{-10}^{+1.1}$ & CV? & $\ldots$ & DH \\
\hline $114 \ldots \ldots \ldots \ldots$ & $002419.3-720334$ & 002419.3640 .004 & $\begin{array}{llll}-72 & 03 & 34.78 & 0.02\end{array}$ & 101 & $506.4_{-22.5}^{+24.5}$ & $349.5_{-18.7}^{+20.1}$ & $173.3_{-13.1}^{+14.9}$ & $29.5_{-1.3}^{+1.4}$ & $18.3_{-0.9}^{+0.9}$ & $\ldots$ & $\ldots$ & DY \\
\hline $24 \ldots \ldots \ldots \ldots$ & $002407.3-720449$ & 002407.3890 .005 & -720449.630 .02 & 9 & $411.3_{-20.2}^{+22.3}$ & $296.1_{-17.2}^{+18.6}$ & $119.9_{-10.9}^{+12.1}$ & $31.8_{-1.6}^{+1.7}$ & $22.0_{-1.1}^{+1.2}$ & $\mathrm{AB} ?$ & & DHYc \\
\hline $15 \ldots \ldots \ldots \ldots$ & $002408.4-720500$ & 002408.4750 .005 & $\begin{array}{llll}-72 & 0500.30 & 0.02\end{array}$ & 16 & $370.1_{-19.2}^{+21.2}$ & $99.5_{-9.9}^{+11.3}$ & $321.1_{-17.9}^{+19.7}$ & $24.5_{-1.2}^{+1.3}$ & $9.9_{-0.6}^{+0.7}$ & $\mathrm{CV}$ & $\ldots$ & $\ldots$ \\
\hline $20 \ldots \ldots \ldots \ldots \ldots$ & $002407.9-720454$ & 002407.9240 .005 & -720454.760 .02 & 12 & $366.9_{-19.1}^{+12.2}$ & $238.8_{-15.4}^{+16.9}$ & $132.7_{-11.5}^{+13.9}$ & $19.6_{-1.0}^{+1.2}$ & $14.0_{-0.8}^{+0.6}$ & CV? & & \\
\hline $122 \ldots \ldots \ldots \ldots$ & $002403.8-720621$ & 002403.8350 .005 & 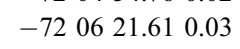 & 89 & $349.6_{-18.7}^{-190.1}$ & $222.6_{-14.9}^{+15.4}$ & $140.1_{-11.8}^{+11.6}$ & $20.2_{-1.1}^{+1.2}$ & $12.6_{-0.7}^{+0.8}$ & $\mathrm{CV}$ & $\cdots$ & $\mathrm{D}$ \\
\hline $117 \ldots \ldots \ldots \ldots$ & $002413.7-720302$ & 002413.7930 .007 & $\begin{array}{llll}-72 & 03 & 02.17 & 0.02\end{array}$ & 116 & $320.5_{-17.9}^{+20.0}$ & $203.3_{-14.2}^{+15.9}$ & $125.2_{-11.1}^{+11.8}$ & $18.7_{-1.0}^{+1.2}$ & $12.2_{-0.7}^{+0.8}$ & $\ldots$ & $\ldots$ & - \\
\hline $44 \ldots \ldots \ldots \ldots \ldots$ & $002403.6-720459$ & 002403.6830 .005 & -720459.060 .03 & 10 & $305.0_{-17.4}^{+19.5}$ & $184.8_{-13.6}^{+15.2}$ & $131.0_{-11.4}^{+13.1}$ & $19.6_{-1.1}^{+1.2}$ & $11.4_{-0.7}^{+0.8}$ & $\mathrm{CV}$ & & $\mathrm{Y}$ \\
\hline $29 \ldots \ldots \ldots \ldots$ & $002406.0-720449$ & 002406.0570 .005 & $\begin{array}{llll}-72 & 04 & 49.03 & 0.02\end{array}$ & 4 & $264.2_{-16.2}^{+18.4}$ & $179.8_{-13.4}^{+14.9}$ & $95.9_{-9.7}^{+11.6}$ & $19.8_{-1.2}^{+1.4}$ & $11.5_{-0.8}^{+0.8}$ & MSP & MSP-W & \\
\hline $113 \ldots \ldots \ldots \ldots$ & $002425.8-720703$ & 002425.8140 .006 & -720703.530 .03 & 161 & $267.4_{-16.3}^{+18.3}$ & $211.2_{-14.5}^{+16.0}$ & $59.3_{-7.6}^{+9.5}$ & $15.5_{-0.9}^{+1.1}$ & $10.7_{-0.7}^{+0.9}$ & & & $\mathrm{H}$ ? \\
\hline
\end{tabular}

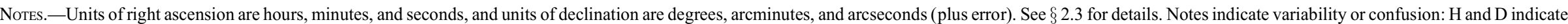

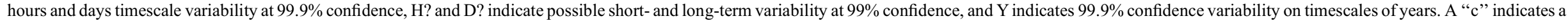

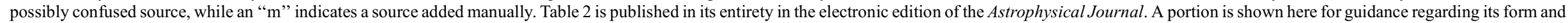
content. 
TABLE 3

47 Tuc Basic X-Ray Source Properties, 2000 Data Set

\begin{tabular}{|c|c|c|c|c|c|c|c|c|c|c|c|c|c|}
\hline \multicolumn{2}{|r|}{ NAMES } & \multicolumn{2}{|c|}{ Position } & \multirow[b]{2}{*}{$\begin{array}{r}f_{\mathrm{PSF}} \\
(5)\end{array}$} & \multirow[b]{2}{*}{$\begin{array}{c}f_{\mathrm{EXP}} \\
(6)\end{array}$} & \multicolumn{3}{|c|}{ Counts } & \multicolumn{2}{|c|}{$L_{\mathrm{X}}$} & \multirow[b]{2}{*}{$\begin{array}{l}\text { ID TYPE } \\
(12)\end{array}$} & \multirow[b]{2}{*}{$\begin{array}{c}\text { OtHER NAMES } \\
\text { (13) }\end{array}$} & \multirow[b]{2}{*}{$\begin{array}{l}\text { Notes } \\
(14)\end{array}$} \\
\hline $\begin{array}{l}\mathrm{W} \\
(1)\end{array}$ & $\begin{array}{c}\text { CXOGlb J } \\
\text { (2) }\end{array}$ & $\begin{array}{c}\alpha \\
(\mathrm{J} 2000.0) \\
(3)\end{array}$ & $\begin{array}{c}\delta \\
(\mathrm{J} 2000.0) \\
(4)\end{array}$ & & & $\begin{array}{l}0.5-4.5 \\
\mathrm{keV} \\
(7)\end{array}$ & $\begin{array}{l}0.5-1.5 \\
\mathrm{keV} \\
(8)\end{array}$ & $\begin{array}{l}1.5-6 \\
\mathrm{keV} \\
(9)\end{array}$ & $\begin{array}{c}0.5-6.0 \\
\left(10^{30} \mathrm{ergs} \mathrm{s}^{-1}\right) \\
(10)\end{array}$ & $\begin{array}{c}0.5-2.5 \\
\left(10^{30} \mathrm{ergs} \mathrm{s}^{-1}\right) \\
(11)\end{array}$ & & & \\
\hline $46 \ldots \ldots \ldots \ldots . .$. & $002403.4-720451$ & 002403.4890 .001 & -720451.920 .01 & 0.96 & 1.00 & $5308.2_{-72.8}^{+74.7}$ & $4403.7_{-66.4}^{+67.8}$ & $904.4_{-30.1}^{+31.4}$ & $1338.0_{-18.4}^{+18.8}$ & $1185.4_{-16.4}^{+16.8}$ & $\mathrm{qLX}$ & $\mathrm{X} 7$ & \\
\hline $58 \ldots \ldots \ldots \ldots \ldots$ & $002400.9-720453$ & 002400.9400 .001 & -720453.180 .01 & 0.96 & 1.00 & $4424.2_{-66.5}^{+68.8}$ & $3573.3_{-59.8}^{+6.4}$ & $854.8_{-29.2}^{+30.1}$ & $1113.6_{-16.7}^{+18.4}$ & $958.9_{-14.6}^{+10.4}$ & $\mathrm{qLX}$ & $\mathrm{X} 5$ & DHY \\
\hline $42 \ldots \ldots \ldots \ldots$ & $002404.2-720458$ & $\begin{array}{llll}00 & 24 & 04.242 & 0.002\end{array}$ & -720457.980 .01 & 0.94 & 1.00 & $2407.3_{-49.0}^{+50.1}$ & $1470.3_{-38.3}^{+39.8}$ & $1165.7_{-34.1}^{+35.9}$ & $684.4_{-13.3}^{+1.9}$ & $442.0_{-10.1}^{+10.5}$ & $\mathrm{CV}$ & $\mathrm{X} 9$ & $\mathrm{H} ? \mathrm{Y}$ \\
\hline $56 \ldots \ldots \ldots \ldots$ & $002402.1-720542$ & 002402.1110 .004 & -720541.950 .02 & 0.96 & 1.00 & $725.8_{-26.9}^{+29.0}$ & $353.4_{-18.8}^{+20.3}$ & $415.3_{-20.4}^{+22.1}$ & $195.2_{-7.0}^{+7.6}$ & $109.9_{-4.5}^{+4.9}$ & $\mathrm{CV}$ & X6 & $\mathrm{Y}$ \\
\hline $27 \ldots \ldots \ldots \ldots$ & $002406.3-720443$ & 002406.3700 .003 & -720442.970 .01 & 0.95 & 0.99 & $727.5_{-26.9}^{+28.9}$ & $562.4_{-23.7}^{+25.1}$ & $194.9_{-13.9}^{+15.4}$ & $193.1_{-7.0}^{+7.0}$ & $162.9_{-6.4}^{+6.8}$ & $\mathrm{CV}$ & $\mathrm{X} 10$ & DH \\
\hline $30 \ldots \ldots \ldots \ldots$ & $002406.0-720456$ & 002405.9910 .004 & -720456.180 .03 & 0.92 & 1.00 & $320.4_{-17.9}^{+20.0}$ & $176.1_{-13.2}^{+14.7}$ & $158.1_{-12.5}^{+14.3}$ & $89.1_{-4.9}^{+5.5}$ & $51.8_{-3.3}^{+3.6}$ & $\mathrm{CV}$ & $\mathrm{X} 19$ & $\mathrm{Y}$ \\
\hline $45 \ldots \ldots \ldots \ldots$ & $002403.7-720423$ & 002403.7560 .005 & -720422.910 .02 & 0.95 & 0.99 & $258.0_{-16.0}^{+18.9}$ & $148.4_{-12.2}^{+13.6}$ & $123.5_{-11.1}^{+12.9}$ & $69.2_{-4.2}^{+4.9}$ & $43.4_{-2.9}^{+3.3 .3}$ & $\mathrm{CV}$ & $\ldots$ & $\ldots$ \\
\hline $1 \ldots \ldots \ldots \ldots$ & $002416.9-720427$ & 002416.9630 .006 & -720427.180 .03 & 0.95 & 0.99 & $249.4_{-15.7}^{+17.9}$ & $131.7_{-11.4}^{+12.9}$ & $133.6_{-11.5}^{+13.3}$ & $67.4_{-4.1}^{+4.7}$ & $41.7_{-2.9}^{+3.2}$ & $\mathrm{CV}$ & $\ldots$ & $\mathrm{Y}$ \\
\hline $2 \ldots \ldots \ldots \ldots \ldots$ & $002415.8-720436$ & 002415.8760 .006 & -720436.390 .03 & 0.95 & 0.99 & $196.3_{-14.0}^{+16.1}$ & $101.6_{-10.0}^{+11.6}$ & $109.6_{-10.4}^{+11.3 .3}$ & $53.7_{-3.7}^{+4.3}$ & $30.7_{-2.5}^{+2.8}$ & $\mathrm{CV}$ & $\mathrm{X} 13$ & DY \\
\hline $64 \ldots \ldots \ldots \ldots$ & $002357.6-720502$ & 002357.6670 .007 & -720501.950 .03 & 0.95 & 0.99 & $170.8_{-13.0}^{+15.0}$ & $93.1_{-9.6}^{+11.0}$ & $91.5_{-9.5}^{+11.4}$ & $47.4_{-3.5}^{+4.1}$ & $28.3_{-2.4}^{+2.7}$ & $\mathrm{AB}$ & $\ldots$ & \\
\hline $47 \ldots \ldots \ldots \ldots$ & $002403.4-720505$ & 002403.4430 .007 & $\begin{array}{llll}-72 & 0505.22 & 0.03\end{array}$ & 0.95 & 0.99 & $168.9_{-12.9}^{+15.1}$ & $99.7_{-9.9}^{+11.5}$ & $72.8_{-8.5}^{+10.2}$ & $44.1_{-3.3}^{+3.9}$ & $31.5_{-2.5}^{+2.9}$ & $\mathrm{AB}$ & $\ldots$ & DY \\
\hline $25 \ldots \ldots \ldots \ldots$ & $002407.1-720545$ & 002407.1220 .008 & -720545.720 .04 & 0.95 & 1.00 & $135.5_{-11.6}^{+13.9}$ & $88.7_{-9.4}^{+10.9}$ & $52.7_{-7.2}^{+9.3}$ & $39.8_{-3.3}^{+4.0}$ & $27.6_{-2.5}^{+2.9}$ & $\mathrm{CV}$ & $\mathrm{X} 11$ & \\
\hline $8 \ldots \ldots \ldots \ldots$ & $002410.7-720425$ & $\begin{array}{llll}00 & 24 & 10.746 & 0.010\end{array}$ & -720425.730 .04 & 0.95 & 0.99 & $72.2_{-8.4}^{+10.5}$ & $11.6_{-3.3}^{+5.4}$ & $82.5_{-9.0}^{+10.9}$ & $23.9_{-2.4}^{+3.1 .3}$ & $5.6_{-1.0}^{+1.3}$ & $\mathrm{CV}$ & $\ldots$ & $\mathrm{Y}$ \\
\hline $15 \ldots \ldots \ldots \ldots$ & $002408.4-720500$ & 002408.4590 .009 & -720500.290 .04 & 0.92 & 0.94 & $86.6_{-9.2}^{+11.4}$ & $19.1_{-4.3}^{+5.9}$ & $77.4_{-8.7}^{+10.6}$ & $27.1_{-2.7}^{+3.4}$ & $9.2_{-1.3}^{+1.0}$ & $\mathrm{CV}$ & $\ldots$ & $\ldots$ \\
\hline $17 \ldots \ldots \ldots \ldots$ & $002408.3-720431$ & 002408.3110 .008 & -720431.400 .04 & 0.92 & 0.99 & $95.2_{-9.7}^{+11.8}$ & $72.5_{-8.5}^{+10.0}$ & $23.6_{-4.8}^{+6.7}$ & $25.5_{-2.6}^{+3.2}$ & $22.0_{-2.3}^{+2.8}$ & qLX? & $\ldots$ & $\ldots$ \\
\hline $16 \ldots \ldots \ldots \ldots$ & $002408.2-720435$ & 002408.2870 .009 & -720435.670 .06 & 0.92 & 1.00 & $90.9_{-9.5}^{+11.7}$ & $52.3_{-7.2}^{+8.7}$ & $43.6_{-6.5}^{+8.5}$ & $25.4_{-2.6}^{+3.2}$ & $16.9_{-2.0}^{+2.4}$ & CV? & $\cdots$ & $\mathrm{cY}$ \\
\hline $23 \ldots \ldots \ldots \ldots$ & $002407.8-720441$ & 002407.7870 .008 & -720441.530 .05 & 0.75 & 0.99 & $88.4_{-9.3}^{+11.5}$ & $48.5_{-69}^{+8.5}$ & $43.8_{-65}^{+8.4}$ & $30.5_{-3.9}^{+3.9}$ & $18.7_{-2.1}^{+2.6}$ & $\mathrm{AB}$ & $\ldots$ & $\mathrm{Y}$ \\
\hline $32 \ldots \ldots \ldots \ldots \ldots$ & $002405.6-720449$ & 002405.6470 .011 & -720449.130 .04 & 0.79 & 0.99 & $71.5_{-8.4}^{+10.6}$ & $49.9_{-7.0}^{+6.9}$ & $23.5_{-4.7}^{+6.7}$ & $23.0_{-2.7}^{+3.4}$ & $18.3_{-2.3}^{+2.8}$ & CV? & $\ldots$ & $\mathrm{c}$ \\
\hline $120 \ldots \ldots \ldots$ & $002411.0-720620$ & 002411.0990 .012 & -720619.930 .04 & 0.95 & 1.00 & $67.3_{-8.1}^{+10.4}$ & $38.7_{-6.2}^{+7.7}$ & $34.6_{-5.8}^{+7.8}$ & $18.8_{-2.2}^{+2.8}$ & $11.6_{-1.5}^{+2.0}$ & $\mathrm{CV}$ & $\ldots$ & $\ldots$ \\
\hline $53 \ldots \ldots \ldots \ldots$ & $002402.5-720511$ & 002402.5170 .010 & -720511.250 .05 & 0.95 & 1.00 & $62.3_{-7.8}^{+10.1}$ & $33.8_{-5.8}^{+0.4}$ & $32.2_{-5.6}^{+7.0}$ & $16.9_{-2.0}^{+2.7}$ & $11.1_{-1.5}^{+1.9}$ & $\mathrm{CV}$ & $\ldots$ & $\mathrm{Y}$ \\
\hline $117 \ldots \ldots \ldots$ & $002413.7-720302$ & 002413.7780 .010 & $\begin{array}{llll}-72 & 03 & 02.13 & 0.07\end{array}$ & 0.95 & 0.87 & $54.5_{-7.3}^{+9.5}$ & $37.8_{-6.1}^{+7.7}$ & $22.7_{-4.6}^{+6.7}$ & $18.0_{-2.3}^{+3.0}$ & $11.2_{-1.6}^{+2.1}$ & $\ldots$ & $\ldots$ & $\ldots$ \\
\hline $21 \ldots \ldots \ldots$ & $002407.7-720527$ & 002407.7440 .012 & -720527.150 .05 & 0.92 & 0.99 & $59.5_{-7.6}^{+9.9}$ & $36.7_{-6.0}^{+0.6}$ & $23.7_{-4.8}^{+6.0}$ & $16.2_{-2.0}^{+2.7}$ & $12.1_{-1.7}^{+2.1 .0}$ & $\mathrm{CV}$ & $\ldots$ & $\ldots$ \\
\hline $113 \ldots \ldots \ldots$ & $002425.8-720703$ & 002425.8440 .018 & $\begin{array}{llll}-72 & 0703.52 & 0.09\end{array}$ & 0.94 & 0.99 & $59.4_{-7.6}^{+9.8}$ & $45.7_{-6.7}^{+8.0}$ & $13.7_{-3.6}^{+5.6}$ & $15.7_{-2.0}^{+2.6}$ & $12.3_{-1.6}^{+2.1}$ & & $\ldots$ & \\
\hline $20 \ldots \ldots \ldots \ldots$ & $002407.9-720454$ & 002407.9140 .011 & -720454.780 .05 & 0.92 & 1.00 & $56.3_{-7.4}^{+9.6}$ & $36.9_{-6.0}^{+7.7}$ & $20.3_{-4.4}^{+6.6}$ & $15.2_{-2.0}^{+2.6}$ & $10.9_{-1.5}^{+1.9}$ & CV? & $\cdots$ & $\mathrm{H} ?$ \\
\hline $125 \ldots \ldots \ldots$ & $002353.9-720350$ & 002353.9590 .015 & -720350.120 .06 & 0.95 & 0.93 & $50.8_{-7.0}^{+9.4}$ & $38.9_{-6.2}^{+6.7}$ & $11.9_{-3.3}^{+5.4}$ & $31.7_{-4.4}^{+5.9}$ & $30.4_{-4.3}^{+1.5}$ & qLX? & X4 & \\
\hline $51 \ldots \ldots \ldots \ldots$ & $002402.8-720449$ & $002402.800 \quad 0.014$ & -720449.040 .06 & 0.82 & 0.99 & $46.3_{-6.7}^{+9.0}$ & $33.7_{-5.7}^{+6.3}$ & $14.6_{-3.7}^{+5.8}$ & $14.5_{-2.0}^{+2.4}$ & $9.8_{-1.5}^{+1.9}$ & $\mathrm{CV}$ & $\ldots$ & $\mathrm{Y}$ \\
\hline $122 \ldots \ldots \ldots \ldots$ & $002403.8-720621$ & 002403.8460 .015 & -720621.660 .08 & 0.95 & 0.99 & $44.4_{-6.6}^{+8.9}$ & $27.7_{-5.2}^{+6.8}$ & $19.6_{-4.3}^{+6.4}$ & $12.2_{-1.7}^{+2.4}$ & $8.6_{-1.5}^{+2.0}$ & $\mathrm{CV}$ & $\ldots$ & $\ldots$ \\
\hline $29 \ldots \ldots \ldots \ldots$ & $002406.0-720449$ & 002406.0470 .013 & -720448.960 .07 & 0.77 & 1.00 & $42.7_{-6.4}^{+8.0}$ & $29.1_{-5.3}^{+6.9}$ & $17.6_{-4.1}^{+6.3}$ & $14.9_{-2.1}^{+3.0}$ & $9.5_{-1.6}^{+2.1}$ & MSP & MSP-W & $\mathrm{c}$ \\
\hline $37 \ldots \ldots \ldots \ldots$ & $002404.9-720451$ & 002404.9770 .018 & -720451.300 .09 & 0.92 & 1.00 & $39.8_{-6.2}^{+8.5}$ & $24.6_{-4.9}^{+6.5}$ & $17.0_{-4.0}^{+6.1}$ & $11.1_{-1.7}^{+2.4}$ & $7.1_{-1.2}^{+1.6}$ & $\mathrm{qLX}$ & $\ldots$ & $\mathrm{Y}$ \\
\hline $14 \ldots \ldots \ldots \ldots$ & $002408.7-720507$ & 002408.7600 .014 & $\begin{array}{llll}-72 & 0507.55 & 0.08\end{array}$ & 0.92 & 0.93 & $40.1_{-6.2}^{+8.5}$ & $27.5_{-5.2}^{+6.8}$ & $12.6_{-3.5}^{+5.5}$ & $11.3_{-1.7}^{+2.5}$ & $7.7_{-1.3}^{+1.8}$ & $\mathrm{AB}$ & $\ldots$ & $\ldots$ \\
\hline
\end{tabular}

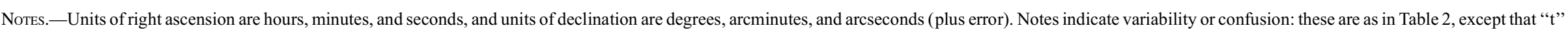

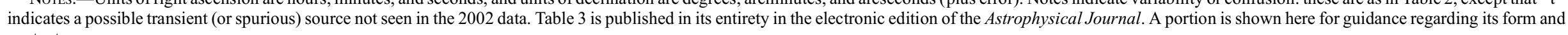
content. 


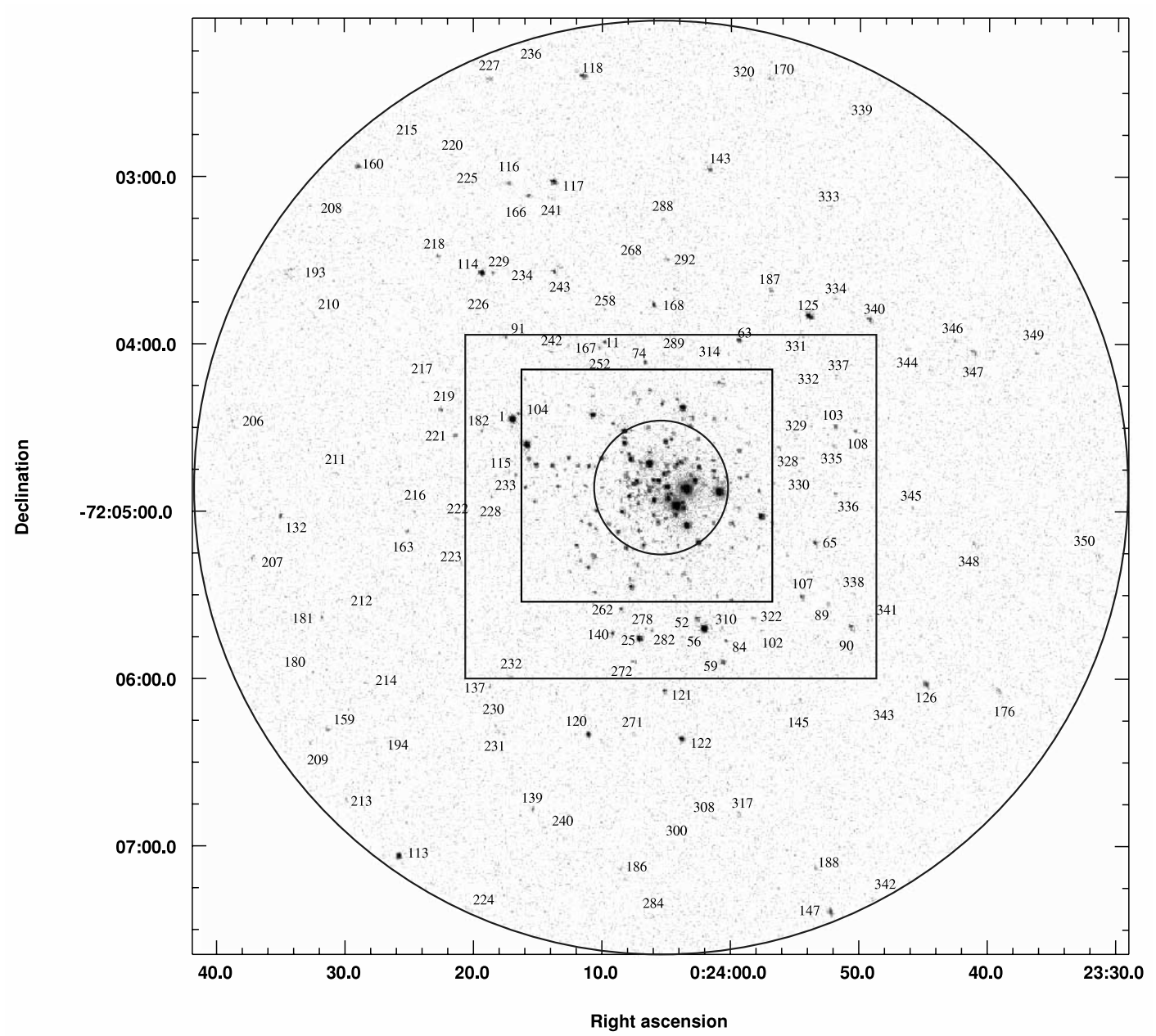

FIG. 1.-Combined $20020.3-6 \mathrm{keV}$ Chandra data of 47 Tuc, out to the half-mass radius. Circles indicate the core (inner) and half-mass radii. Two boxes indicate the region analyzed in G01a and the (smaller) inset region shown in more detail in Fig. 2. The 47 Tuc X-ray sources identified in this paper outside the inset region are labeled with their shorthand W numbers (see Table 2).

harder energy band images confirmed that the low column density to 47 Tuc and nonaccreting nature of the faintest sources (ABs and MSPs) make the faintest sources quite soft. The wavdetect tool is often inefficient at separating two or more point sources less than $3^{\prime \prime}$ apart (as noted by Feigelson et al. 2002). Several faint sources within a few arcseconds of brighter sources can be clearly picked out by eye but are not identified by wavdetect. We add 10 sources identified by eye to the 2002 results and 12 sources to the 2000 results, without reference to any information about possible counterparts at other wavelengths. We also check our source list for any spurious detections of cosmic rays missed by acis_detect_afterglow (identified when more than half of the counts from a faint source fall within a $30 \mathrm{~s} \mathrm{time}$ interval, with each event decreasing in energy from the last). Several such spurious detections are identified and eliminated in the 2000 data, including W88 and W99 from the source list of G01a. ${ }^{10}$ These spurious sources also fail to appear in the 2002 data, while all but three other sources detected in the 2000 data are also detected in the 2002 data set. The recovery of nearly all the detected 2000 sources in the 2002 data provides an upper limit to wavdetect's spurious source detection rate, implying that probably no more than two or three of the 2002 sources are spurious.

\footnotetext{
10 The acis_detect_afterglow algorithm seems to detect all cosmic rays on the back-illuminated ACIS-S3 chip, unlike on the ACIS-I chips.
}

We combine the results from the two energy bands to make independent source lists for the 2000 and 2002 observations, given in Tables 2 and 3. A total of 146 sources are detected in this way in the 2000 observations, while 300 sources are detected in the 2002 observations. A total of 143 of the sources are clearly detected in both observations, while only three of the sources from the 2000 observations are not detected in the 2002 observations. Of these three, two (W149 and W172) show only $2-3$ counts and may not be real sources, while W68 (identified with an optical AB counterpart; Edmonds et al. 2003a) seems to have disappeared. We retain the $\mathrm{W}$ numbering scheme of G01a, as extended in Grindlay et al. (2002) and Edmonds et al. (2003a, 2003b), for the 2000 observations, extending it to cover first the remaining 2000 sources and then the additional 2002 sources. We also generate IAU-registered positional source names, in the form CXOGlb J[xxxx.x-xxxxx]. We list the sources in order of decreasing total counts in both tables. Because of the iterative nature of source list construction over several papers, the W numbering scheme does not follow clear patterns and has holes (numbers that do not correspond to real sources within our chosen field).

In Figures 1 and 2 we show the raw unbinned photon count data from the combined 2002 observations we used for source detection, in the energy range $0.3-6 \mathrm{keV}$. The $\mathrm{W}$ source numbers are indicated in both figures. In Figure 3 we show a representativecolor image of the 2002 data made from the $0.3-1.2,1.2-2$, and 


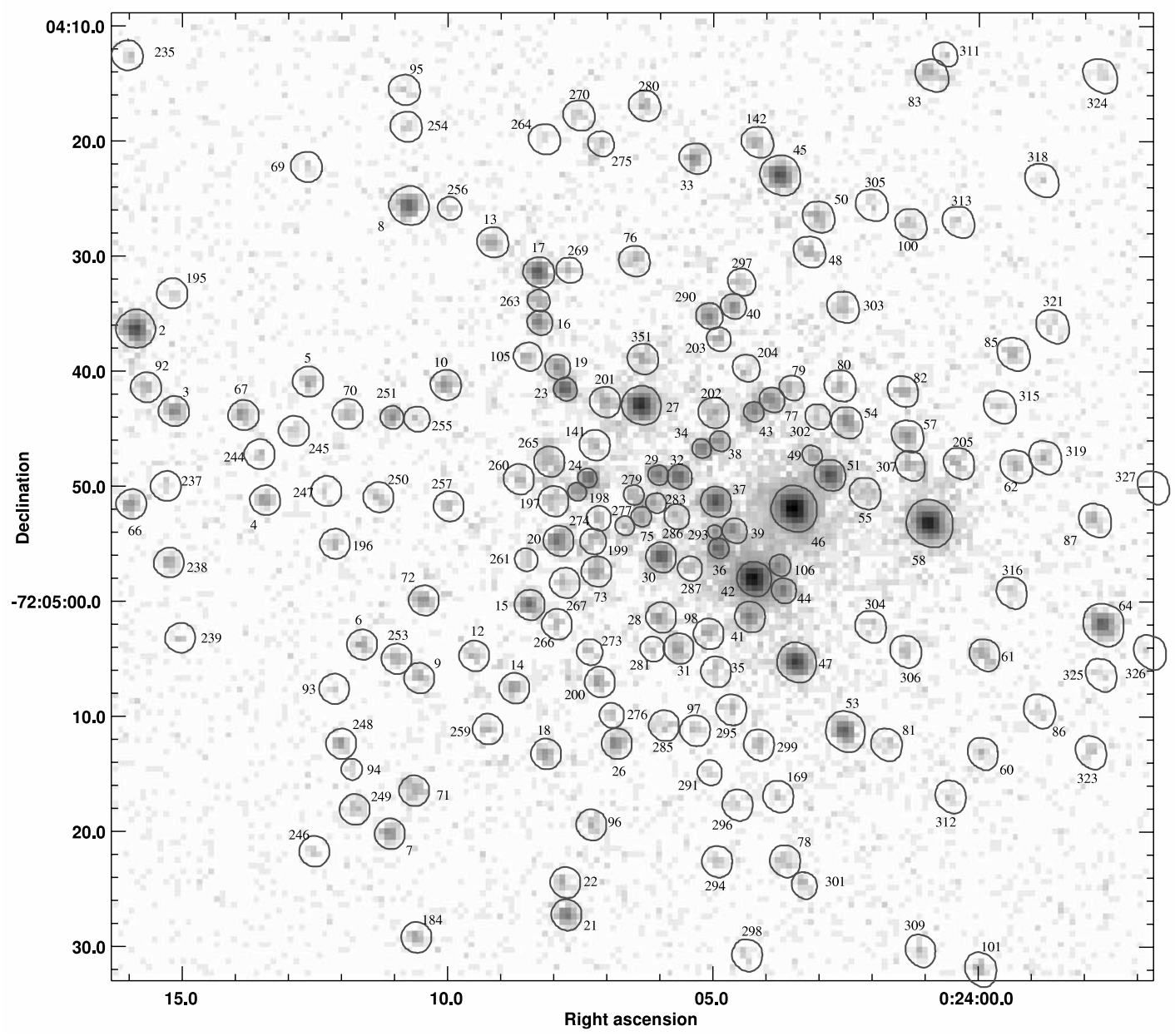

FIG. 2.-Combined $20020.3-6 \mathrm{keV}$ Chandra data of $47 \mathrm{Tuc}$, within the inset box of Fig. 1. The polygons used for extracting source events, photometry, and spectra (from the first $2002 \mathrm{ObsID}$; regions for other ObsIDs are similar) are indicated, and labels indicate the W numbers of the X-ray sources discussed in this paper. The gray scale is logarithmic from 0 to 8306 counts.

2-6 keV exposure map-corrected data, overbinned and smoothed on a scale of 0 ".74.

We align our absolute astrometry with the 17 MSPs in 47 Tuc that have positions previously determined through radio timing studies (or HST optical follow-up; Edmonds et al. 2002a) to milliarcsecond levels (Camilo et al. 2000; Freire et al. 2001, 2003). We find that each of the MSPs with radio timing (or optical) positions is detected, with only the close pairs 47 Tuc G and I (separation 0.12 ) and F and S (separation 0.74) unresolved. (We do not attempt to separate their emission in this work; detailed analysis of the MSP X-ray emission is performed in S. Bogdanov et al. [2005, in preparation].) This procedure has been described by G01a and Edmonds et al. (2001, 2003a) for the 2000 Chandra observations, where six cleanly detected MSPs were used to boresight the Chandra frame to the international celestial reference frame. We repeat this procedure for the 2002 observations. The rms deviations for 14 MSPs (excluding W77, 47 Tuc F and S) are only 0 ."18 $(\alpha)$ and 0 .'22 $(\delta)$ in the 2002 data. After this work was submitted for publication, a radio timing solution for the position of 47 Tuc $\mathrm{R}$ became available (P. C. Freire et al. 2005, in preparation), which is offset from W198 by only $\Delta \alpha=+0.03, \Delta \delta=-0.01$ and thus a convincing identification.

\subsection{Optical Identifications}

Most of the identifications for objects discussed in this work rely on the optical identification results of Edmonds et al. (2003a, 2003b), using the 2000 Chandra data set and HST programs GO-8267 (PI: Gilliland) and GO-7503 (PI: Meylan). We also include an identification by Ferraro et al. (2001) of a bright red star as a candidate counterpart for W54, which Edmonds et al. (2003a) suggest is a possible RS CVn star.

In addition to these identifications, we compare the variable and blue stars of Geffert et al. (1997), CV candidates of Knigge et al. (2002), and binaries of A01 to our data. No additional matches are found with the star lists of Geffert et al. (1997) or CV candidates of Knigge et al. (2002), but the binary lists of A01 provide a large number of plausible candidate counterparts, as we describe below.

Numerous binaries in the GO-8267 HST data have already been identified as X-ray sources by Edmonds et al. (2003a). We use the astrometric plate solutions of Edmonds et al. (2003a) to align each HST WFPC2 chip to our Chandra frame. Then we compared the A01 binaries to the positions of our Chandra sources and identified those binaries that fell within $5 \sigma$ in both right ascension and declination as likely matches (where the errors are the Chandra wavdetect errors added in quadrature with the systematic errors in the plate solutions). In this way we recovered every A01 binary previously matched to an X-ray source and identified 32 new possible matches. These include an alternative match for W93, the optical variable WF4-V02, that appears closer to the Chandra position than the marginal ID identified by Edmonds et al. (2003a). The new matches are listed in Table 4, with their offsets from the aligned Chandra 


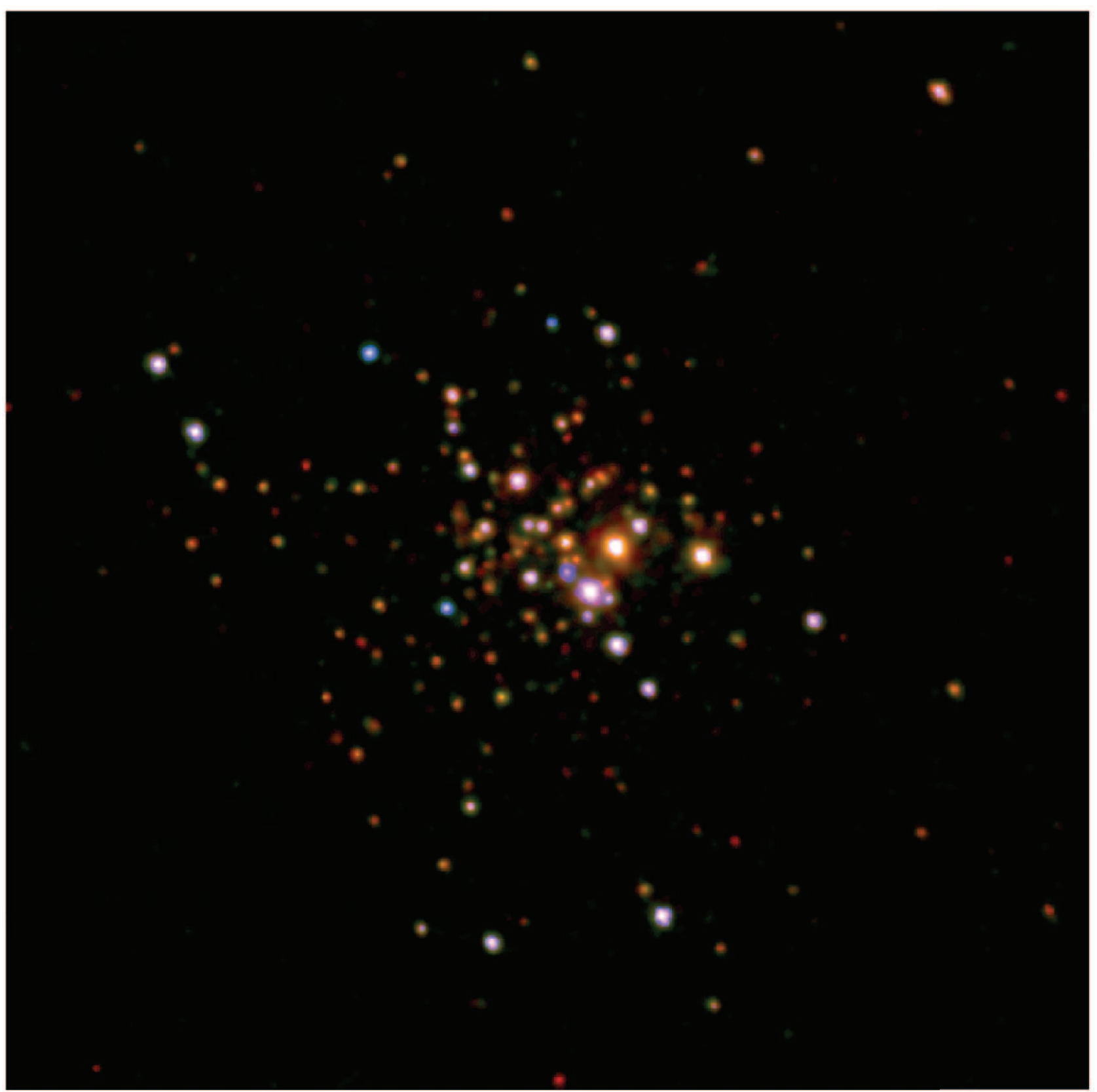

FIg. 3.-Combined 2002 exposure-corrected Chandra data of the core region of 47 Tuc (148" square). This representative-color image was constructed from a $0.3-$ $1.2 \mathrm{keV}$ image (red), a $1.2-2 \mathrm{keV}$ image (green), and a $2-6 \mathrm{keV}$ image (blue). All images were overbinned by a factor of 2 , smoothed using the CIAO tool csmooth, and then combined.

positions and classifications from A01. The most discrepant new match we discuss is PC1-V21 with W287, at 1."01. This is a $2.87 \sigma$ discrepancy, with a chance coincidence probability of roughly $2.9 \%$.

The rms total offsets for the new A01 Chandra matches are $0.42,0.21,0.32$, and 0.21 for the PC, WF2, WF3, and WF4 chips, respectively $(0,23$ for the PC chip when W287 is excluded). We note that the Chandra ACIS-S array is likely to have different small systematic astrometric errors (owing to its different geometry) compared to the ACIS-I array. Offseting the Chandra positions by $5^{\prime \prime}$ in right ascension and declination produced two spurious matches in four trials, suggesting an even probability that 1 of our 32 new matches is spurious. Figure 4 plots optical color-magnitude diagrams (CMDs), including the new A01 matches and the Edmonds et al. (2003a) matches, from the GO-8267 HST data. It is clear that the new A01 matches are similar to the previously identified ABs, but optically fainter on average (mean $V$ magnitudes 19.3 vs. 18.7, respectively). In the remainder of this paper we include W287 among the candidate $\mathrm{ABs}$, while the other 31 new identifications from $\mathrm{A} 01$ are included among the confirmed ABs.

We note that some variable stars listed by A01 appear to the eye to be faint and/or confused X-ray sources that were missed by wavdetect. For completeness we list these stars here, although their selection is subjective and may be biased. Therefore, we do not discuss them in the rest of this paper. They are PC1-V07, PC1-V20, WF2-V47, PC1-V22, PC1-V28, PC1-V31, PC1-V33, PC1-V35, PC1-V37, PC1-V38, WF2-V14, WF2-V20, WF3-V21, and WF4-V09. These stars are similar in their optical properties to the stars matched to detected X-ray sources above. We also note 
TABLE 4

New Optical Counterpart Identifications

\begin{tabular}{|c|c|c|c|c|c|c|c|}
\hline W & CXOGlb J & A01 Name & $\begin{array}{c}\Delta \alpha \\
(\operatorname{arcsec})\end{array}$ & $\begin{array}{c}\Delta \delta \\
(\operatorname{arcsec})\end{array}$ & Separation/Error & Type & $\begin{array}{l}\text { Period } \\
\text { (days) }\end{array}$ \\
\hline $287 \ldots \ldots \ldots \ldots \ldots$ & $002405.4-720457$ & PC1-V21 & -0.665 & 0.765 & 2.87 & N.Ecl. S.Det. & 0.39 \\
\hline $266 \ldots \ldots \ldots \ldots \ldots$ & $002407.9-720502$ & PC1-V10 & 0.165 & 0.01 & 1.42 & W UMa S.Det. & 0.43 \\
\hline $299 \ldots$ & $002404.1-720512$ & PC1-V30 & 0.101 & 0.023 & 1.08 & BY Dra & 0.80 \\
\hline $267 \ldots$ & $002407.8-720458$ & PC1-V04 & 0.036 & 0.023 & 0.41 & W UMa Con. & 0.33 \\
\hline $286 \ldots$ & $002405.6-720452$ & PC1-V32 & 0.073 & 0.162 & 2.00 & BY Dra & 1.64 \\
\hline 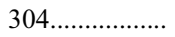 & $002402.0-720502$ & PC1-V42 & 0.345 & 0.382 & 3.43 & BY Dra & 0.52 \\
\hline $274 \ldots \ldots \ldots \ldots \ldots$ & $002407.1-720452$ & PC1-V34 & -0.065 & -0.049 & 0.97 & BY Dra & 1.38 \\
\hline $273 \ldots \ldots \ldots \ldots \ldots$ & $002407.3-720504$ & PC1-V18 & -0.092 & 0.162 & 1.48 & W UMa Con. & 0.30 \\
\hline $289 \ldots \ldots$ & $002405.1-720404$ & WF2-V02 & 0.327 & -0.211 & 1.71 & Ecl. Bin. & 3.97 \\
\hline $256 \ldots \ldots \ldots \ldots \ldots$ & $002410.0-720426$ & WF2-V21 & -0.072 & -0.227 & 0.67 & BY Dra & 1.50 \\
\hline $275 \ldots \ldots \ldots \ldots \ldots$ & $002407.1-720420$ & WF2-V22 & -0.005 & -0.021 & 0.20 & BY Dra & 0.55 \\
\hline $258 \ldots$ & $002409.8-720348$ & WF2-V04 & 0.082 & 0.143 & 0.88 & W UMa Con. & 0.22 \\
\hline $280 \ldots$ & $002406.3-720416$ & WF2-V06 & -0.030 & -0.009 & 0.32 & W UMa S.Det. & 0.37 \\
\hline $257 \ldots \ldots \ldots \ldots \ldots$ & $002410.0-720451$ & WF2-V07 & -0.113 & 0.084 & 1.21 & W UMa S.Det. & 0.29 \\
\hline $242 \ldots \ldots \ldots \ldots \ldots$ & $002414.0-720403$ & WF2-V11 & -0.011 & 0.185 & 1.05 & BY Dra & 0.84 \\
\hline $244 \ldots \ldots \ldots \ldots \ldots$ & $002413.5-720447$ & WF2-V12 & 0.276 & -0.004 & 2.08 & BY Dra & 1.18 \\
\hline $243 \ldots \ldots \ldots \ldots \ldots$ & $002413.7-720334$ & WF2-V32 & -0.121 & -0.210 & 3.40 & Red Str. & 9.2 \\
\hline $251 \ldots \ldots \ldots \ldots \ldots$ & $002411.0-720444$ & WF2-V15 & -0.028 & 0.044 & 0.89 & BY Dra & 1.29 \\
\hline $211 \ldots \ldots \ldots \ldots \ldots$ & $002430.7-720446$ & WF3-V02 & 0.417 & -0.066 & 1.90 & Ecl. Bin. & 2.31 \\
\hline $238 \ldots \ldots \ldots \ldots \ldots$ & $002415.2-720456$ & WF3-V03 & 0.108 & 0.110 & 2.07 & W UMa Con. & 0.33 \\
\hline $233 \ldots \ldots \ldots \ldots \ldots$ & $002416.7-720447$ & WF3-V12 & 0.113 & 0.371 & 2.91 & BY Dra & 1.78 \\
\hline $237 \ldots \ldots \ldots \ldots \ldots$ & $002415.3-720450$ & WF3-V22 & 0.127 & 0.440 & 2.85 & BY Dra & 0.89 \\
\hline $228 \ldots \ldots \ldots \ldots \ldots$ & $002418.6-720455$ & WF3-V04 & 0.118 & -0.027 & 0.90 & W UMa Con. & 0.25 \\
\hline $222 \ldots \ldots \ldots \ldots \ldots$ & $002421.2-720503$ & WF3-V14 & 0.112 & 0.286 & 1.71 & BY Dra & 5.16 \\
\hline 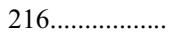 & $002424.6-720500$ & WF3-V15 & 0.207 & -0.029 & 1.31 & BY Dra & 0.44 \\
\hline $239 \ldots \ldots \ldots \ldots \ldots$ & $002415.0-720503$ & WF3-V16 & 0.172 & 0.279 & 2.01 & BY Dra & 2.50 \\
\hline $262 \ldots \ldots \ldots \ldots \ldots$ & $002408.5-720535$ & WF4-V04 & -0.113 & 0.060 & 1.97 & Ecl. Bin. & 4.92 \\
\hline $278 \ldots \ldots \ldots \ldots \ldots$ & $002406.6-720542$ & WF4-V10 & -0.125 & 0.235 & 1.92 & BY Dra & 2.62 \\
\hline $93 \ldots \ldots \ldots \ldots \ldots \ldots$ & $002412.1-720507$ & WF4-V02 & 0.036 & -0.214 & 1.02 & Ecl. Bin. & 4.19 \\
\hline $246 \ldots \ldots \ldots \ldots \ldots$ & $002412.5-720521$ & WF4-V07 & -0.212 & -0.210 & 1.54 & BY Dra & 1.44 \\
\hline $271 \ldots \ldots \ldots \ldots \ldots . . . . . .$. & $002407.5-720620$ & WF4-V08 & -0.151 & 0.069 & 0.84 & BY Dra & 2.04 \\
\hline $248 \ldots \ldots \ldots \ldots \ldots$ & $002412.0-720512$ & WF4-V18 & 0.022 & -0.134 & 2.18 & Red Str. & 5.90 \\
\hline
\end{tabular}

Notes.-Separations $(\Delta \alpha$ and $\Delta \delta)$ are defined as Chandra minus (shifted) HST positions. Separation/Error denotes the right ascension and declination separations divided by the Chandra wavdetect errors plus systematic HST plate solution errors, added in quadrature. Type indicates classification by A01 (on the basis of light curves) as detached eclipsing binaries, BY Dra variables, W UMa contact or semidetached systems, noneclipsing semidetached systems, or red stragglers. Periods are from A01.

that there is no evidence for X-ray emission from the seven unusual "blue variables" discussed by A01 and Edmonds et al. (2003a, 2003b); 90\% confidence upper limits for all but PC1V36 (which lies in a confused region) are $<1.4 \times 10^{29} \mathrm{ergs} \mathrm{s}^{-1}$.

A total of 153 X-ray sources in the 2002 data set lie within the GO-8267 HST field of view. Of these X-ray sources, 3 are identified (by X-ray spectral fitting; see below) as qLMXBs, 12 are identified as $13 \mathrm{MSPs}^{11}$ by matching X-ray and radio positions, 15 are optically identified as CVs, and 57 are optically identified as ABs. ${ }^{12}$ Only $66 \mathrm{X}$-ray sources remain unidentified in this $H S T$ field, only $43 \%$ of the total. Further $H S T$ identifications of optical counterparts, both from the archival HST data sets and from our new simultaneous HST ACS $B, R$, and $\mathrm{H} \alpha$ data, are in progress. Details of the astrometric solution linking the new optical identifications, all of the previously identified counterparts, and new optical counterparts will be presented in M. van den Berg et al. (2005, in preparation).

\subsection{Source Extraction}

For source position improvement, photometry, and extraction of spectra and light curves, we used the IDL (ver. 5.4) tool

11 MSPs G and I, 0".12 apart, are unresolved in X-rays.

$12 \mathrm{~W} 68$, an AB from the 2000 data, is not detected in the 2002 data.
ACIS_EXTRACT (Broos et al. 2002), ${ }^{13}$ which uses CIAO and FTOOLS ${ }^{14}$ tools, ds9 display capability, ${ }^{15}$ and the TARA ${ }^{16}$ IDL software. All spectral fitting was done in XSPEC (Arnaud 1996), version 11.2, much of it within the ACIS_EXTRACT package using scripts written by K. Getman. Our source extraction procedure generally follows the methods of Feigelson et al. (2002) and Muno et al. (2003).

The ACIS_EXTRACT source extraction process begins by calculating region files for each observation designed to match a user-specified contour level of the ACIS point-spread function (PSF), calculated at that position with the CIAO tool mkpsf. For most of our sources, we specified a 90\% encircled energy contour (evaluated at $1.5 \mathrm{keV}$, since most of our sources are relatively soft), due to severe crowding in the central regions. We increased this contour to $95 \%$ for the brightest sources and for relatively bright sources several arcminutes from the cluster center and chose smaller regions that did not overlap for sources very close to each other, reducing the contour to $70 \%$ in some

\footnotetext{
13 Ver. 2.7, http://www.astro.psu.edu/xray/docs/TARA/ae_users_guide.html. 14 Ver. 5.2, http://heasarc.gsfc.nasa.gov/docs/software/ftools/ftools_menu html.

${ }^{15}$ Ver. 2, http://hea-www.harvard.edu/saord/ds9

16 See http://www.astro.psu.edu/xray/docs/TARA.
} 

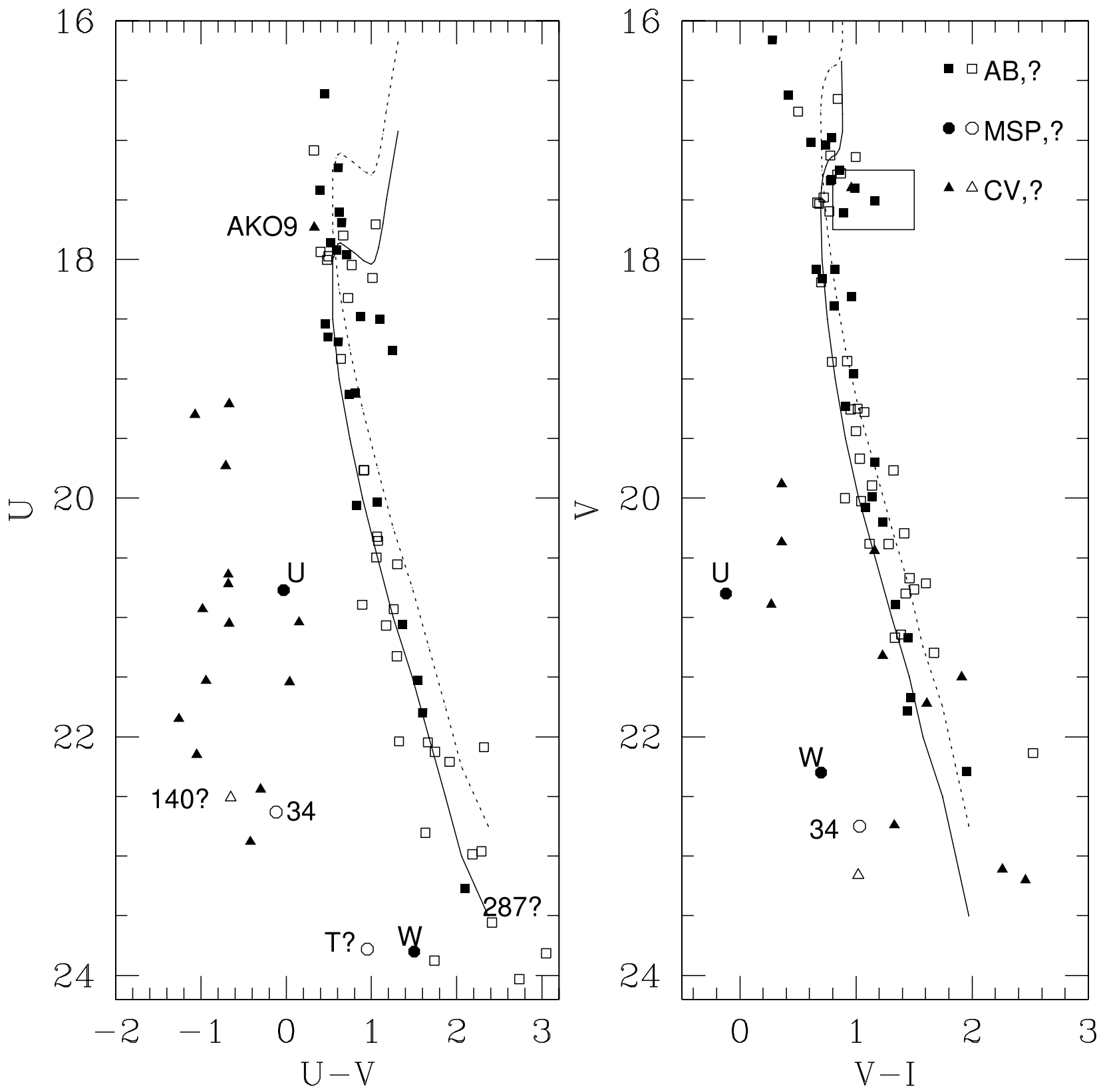

FIg. 4.- $U$ vs. $U-V$ and $V$ vs. $V-I$ CMDs, showing only the optical counterparts for Chandra sources within the GO-8267 HST field of view. New X-ray counterpart ABs from A01 are indicated by open squares, while firm optical counterparts from Edmonds et al. (2003a) are indicated by filled symbols. Four less certain counterparts (see Edmonds et al. 2003a) are also labeled and indicated by open symbols (W34 - either an MSP or a CV—and the possible optical counterparts to W140, 47 Tuc T, and W287). The main-sequence ridge line (solid line) and equal-mass binary sequence (dotted line) are indicated in both CMDs, and a box in the $V$ vs. $V-I$ $\mathrm{CMD}$ indicates the red straggler region from A01. [See the electronic edition of the Journal for a color version of this figure.]

cases. A few sources in the cluster core may still suffer confusion (see $\S 4$ ). The PSF fraction enclosed by the region was calculated at five energies and interpolated for other energies. Event lists, spectra, and light curves were extracted for each source and observation, and response matrices and effective area files (including exposure from multiple CCDs where appropriate) were constructed for each source. Background spectra were extracted for each source using regions sized to include 100 counts and excluding mask regions (generally 1.5 times the size of the $90 \%$ PSF contour) around all detected sources. The effective area functions were created using CIAO version 3.02 and CALDB version 2.26, including the new CALDB ACIS contamination file to correct for the low-energy quantum effi- ciency degradation. ${ }^{17}$ We then corrected for the finite portion of the flux contained within our extraction regions by reducing the effective areas, in an energy-dependent manner (Broos et al. 2002). Composite source and scaled background spectra were then constructed (separately for the 2000 ACIS-I and 2002 ACIS-S images), and appropriately weighted response matrices and effective area files were computed. The total live exposure time for each source was also recorded, and the spectra were grouped to control the placement of the first and last bin, thus constricting the energy range for spectral fitting to $0.5-8.0 \mathrm{keV}$ for ACIS-I data and $0.35-8.0 \mathrm{keV}$ for ACIS-S data.

${ }^{17}$ See http://cxc.harvard.edu/ciao/threads/aciscontam. 
TABLE 5

47 Tuc Supplemental X-Ray Source Properties, 2002 Data Set

\begin{tabular}{|c|c|c|c|c|c|c|c|c|c|c|c|}
\hline \multicolumn{2}{|r|}{ NAMES } & $\begin{array}{c}f_{\mathrm{PSF}} \\
(3)\end{array}$ & $\begin{array}{c}f_{\mathrm{EXP}} \\
(4)\end{array}$ & \multicolumn{4}{|c|}{ Counts } & \multicolumn{4}{|c|}{ HaRdNESS Ratios } \\
\hline $46 \ldots \ldots .$. & $002403.4-720451$ & 0.96 & 1.00 & $33887.0_{-184.1}^{+186.2}$ & $12716.4_{-112.8}^{+114.1}$ & $19329.1_{-139.0}^{+140.4}$ & $1841.5_{-42.9}^{+44.0}$ & $-0.83_{-0.00}^{+0.00}$ & $0.21_{-0.01}^{+0.01}$ & $-0.75_{-0.01}^{+0.01}$ & $5.30_{-0.08}^{+0.08}$ \\
\hline $42 \ldots \ldots \ldots$ & $002404.2-720458$ & 0.94 & 1.00 & $19486.9_{-139.6}^{+1.42 .1}$ & $7562.4_{-87.0}^{+88.3}$ & $7325.6_{-85.6}^{+87.0}$ & $4598.9_{-67.8}^{+69.9}$ & $-0.23_{-0.01}^{+0.00}$ & $-0.02_{-0.01}^{+0.01}$ & $-0.24_{-0.01}^{+0.01}$ & $1.94_{-0.03}^{+0.03}$ \\
\hline $58 \ldots \ldots \ldots$ & $002400.9-720453$ & 0.96 & 1.00 & $11692.0_{-108.1}^{+110.3}$ & $3204.8_{-56.6}^{+57.9}$ & $7652.4_{-87.5}^{+88.8}$ & $834.8_{-28.9}^{+30.8}$ & $-0.80_{-0.01}^{+0.01}$ & $0.41_{-0.01}^{+0.01}$ & $-0.59_{-0.01}^{+0.01}$ & $3.60_{-0.08}^{+0.08}$ \\
\hline $56 \ldots \ldots \ldots$ & $002402.1-720542$ & 0.96 & 1.00 & $2806.8_{-53.0}^{+55.6}$ & $410.3_{-202}^{+21.6}$ & $1587.8_{-398}^{+41.2}$ & $808.7_{-28.4}^{+30.0}$ & $-0.33_{-0.02}^{+0.02}$ & $0.59_{-0.02}^{+0.02}$ & $0.33_{-0.03}^{+0.03}$ & $1.22_{-0.05}^{+0.04}$ \\
\hline $1 \ldots \ldots \ldots$. & $002416.9-720427$ & 0.95 & 1.00 & $1955.0_{-44.2}^{+46.0}$ & $309.6_{-17.6}^{+19.2}$ & $1145.1_{-33.8}^{+35.8}$ & $500.2_{-22.3}^{+23.4}$ & $-0.39_{-0.02}^{+0.02}$ & $0.57_{-0.02}^{+0.02}$ & $0.24_{-0.03}^{+0.03}$ & $1.34_{-0.06}^{+0.06}$ \\
\hline $2 \ldots \ldots \ldots$. & $002415.8-720436$ & 0.95 & 1.00 & $1860.6_{-43.1}^{+45.6}$ & $210.9_{-14.5}^{+15.0}$ & $1039.3_{-32.2}^{+33.0}$ & $610.4_{-24.7}^{+26.3}$ & $-0.26_{-0.02}^{+0.02}$ & $0.66_{-0.02}^{+0.02}$ & $0.49_{-0.03}^{+0.03}$ & $0.89_{-0.04}^{+0.00}$ \\
\hline $51 \ldots \ldots$. & $002402.8-720449$ & 0.89 & 0.98 & $1518.3_{-38.9}^{+41.1}$ & $306.7_{-17.5}^{+18.9}$ & $849.6_{-29.1}^{+30.5}$ & $362.0_{-19.0}^{+20.6}$ & $-0.40_{-0.03}^{+0.03}$ & $0.47_{-0.03}^{+0.02}$ & $0.08_{-0.04}^{+0.03}$ & $1.63_{-0.09}^{+0.04}$ \\
\hline $45 \ldots \ldots \ldots$ & $002403.7-720423$ & 0.95 & 1.00 & $1422.1_{-37.7}^{+40.1}$ & $268.0_{-16.3}^{+17.7}$ & $820.4_{-28.6}^{+30.1}$ & $333.7_{-182}^{+19.8}$ & $-0.42_{-0.03}^{+0.03}$ & $0.51_{-0.03}^{+0.03}$ & $0.11_{-0.04}^{+0.04}$ & $1.50_{-0.09}^{+0.09}$ \\
\hline $125 \ldots \ldots$ & $002353.9-720350$ & 0.95 & 1.00 & $1326.4_{-364}^{+38.8}$ & $667.9_{-25.8}^{+27.3}$ & $564.5_{-23.7}^{+25.0}$ & $94.0_{-9.6}^{+11.3}$ & $-0.71_{-0.03}^{+0.03}$ & $-0.08_{-0.03}^{+0.03}$ & $-0.75_{-0.02}^{+0.03}$ & $4.64_{-038}^{+0.038}$ \\
\hline $64 \ldots \ldots \ldots$ & $002357.6-720502$ & 0.95 & 1.00 & $884.9_{-297}^{+30.1}$ & $205.8_{-14.3}^{+15.3}$ & $434.9_{-208}^{+22.3}$ & $244.2_{-156}^{+17.3}$ & $\begin{array}{l}0.08_{-0.04}^{+0.04} \\
-0.28_{-04}^{+0.04}\end{array}$ & $0.36_{-0.04}^{+0.04}$ & $0.09_{-0.05}^{+0.03}$ & $1.46_{-0.11}^{+0.16}$ \\
\hline $23 \ldots \ldots \ldots$ & $002407.8-720441$ & 0.84 & 1.00 & $\begin{array}{r}-29.7 \\
743.1_{-27.2}^{+29.6}\end{array}$ & $122.1_{-110}^{+14.3}$ & $\begin{array}{l}-20.8 \\
478.1_{-21.8}^{+23.3}\end{array}$ & $142.9^{+13.4}$ & $\begin{array}{l}-0.54^{+0.04} \\
-0.04\end{array}$ & $0.59_{-0.03}^{+0.04}$ & $0.08_{-0.06}^{+0.05}$ & $\begin{array}{l}1.70_{-0.11}^{-11} \\
1.46_{-0.11}^{+0.12}\end{array}$ \\
\hline $53 \ldots \ldots \ldots$ & $002402.5-720511$ & 0.95 & 1.00 & $733.2_{-27.0}^{+29.2}$ & $151.0_{-12.3}^{+11.0}$ & $379.9_{-19.5}^{+21.9}$ & $202.2_{-14.2}^{+11.9}$ & $-0.31_{-0.04}^{+0.03}$ & $0.43_{-0.04}^{+0.03}$ & $0.15_{-0.05}^{+0.06}$ & $1.20_{-0.10}^{+0.11}$ \\
\hline $8 \ldots \ldots \ldots . .$. & $002410.7-720425$ & 0.95 & 1.00 & $711.8_{-26.6}^{+29.1}$ & $17.2_{-4.1}^{+5.3}$ & $264.9_{-16.3}^{+19.5}$ & $429.7_{-20.7}^{+22.4}$ & $0.24_{-0.04}^{+0.04}$ & $0.88_{-0.03}^{+0.04}$ & $0.92_{-0.02}^{+0.02}$ & $0.27_{-0.03}^{+0.03}$ \\
\hline $36 \ldots \ldots \ldots$ & $002404.9-720455$ & 0.78 & 1.00 & $670.8_{-25.9}^{+28.3}$ & $48.0_{-6.9}^{+8.4}$ & $100.8_{-10.0}^{+16.5}$ & $522.0_{-22.8}^{+24.5}$ & $0.68_{-0.03}^{+0.04}$ & $0.36_{-0.08}^{+0.09}$ & $0.83_{-0.02}^{+0.03}$ & $0.21_{-0.02}^{+0.03}$ \\
\hline $32 \ldots \ldots \ldots$ & $002405.6-720449$ & 0.82 & 1.00 & $614.8_{-24.7}^{+27.9}$ & $224.4_{-15.0}^{+6.9}$ & $296.7_{-17.2}^{+10.6}$ & $93.6_{-96}^{+11.3}$ & $-0.52_{-0.04}^{+0.03}$ & $0.14_{-0.04}^{+0.08}$ & $-0.41_{-0.05}^{+0.02}$ & $2.53_{-0.25}^{+0.025}$ \\
\hline $114 \ldots \ldots$ & $002419.3-720334$ & 0.95 & 0.94 & $556.0_{-23.5}^{+26.1}$ & $154.7_{-12.4}^{+15.8}$ & $300.3_{-17.3}^{+17.2}$ & $101.0_{-10.0}^{+11.7}$ & $-0.50_{-0.04}^{+0.04}$ & $0.32_{-0.05}^{+0.04}$ & $-0.21_{-0.06}^{+0.05}$ & $2.02_{-0.20}^{+0.25}$ \\
\hline $24 \ldots \ldots \ldots$ & $002407.3-720449$ & 0.66 & 1.00 & $438.0_{-20.9}^{+23.5}$ & $112.4_{-10.6}^{+12.4}$ & $269.7_{-16.4}^{+17.3}$ & $\begin{array}{l}55.9_{-7.4}^{+9.2} \\
+9.2\end{array}$ & $-0.66_{-0.04}^{+0.04}$ & $0.41_{-0.05}^{+0.05}$ & $-0.34_{-0.08}^{+0.06}$ & $2.47_{-0.29}^{+0.28}$ \\
\hline $15 \ldots \ldots \ldots$ & $002408.4-720500$ & 0.91 & 1.00 & $436.1_{-20.8}^{+20.9}$ & $9.8_{-3.0}^{+4.6}$ & $174.7_{-13.2}^{+16.4}$ & $251.6_{-15.8}^{+17.5}$ & $0.18_{-0.05}^{+0.04}$ & $0.89_{-0.03}^{+0.05}$ & $0.93_{-0.02}^{+0.08}$ & $0.31_{-0.04}^{+0.04}$ \\
\hline $20 \ldots \ldots \ldots$ & $002407.9-720454$ & 0.91 & 1.00 & $385.7_{-19.6}^{+22.1}$ & $68.3_{-8.2}^{+9.7}$ & $252.5_{-15.9}^{+17.3}$ & $64.8_{-8.0}^{+9.7^{\circ}}$ & $-0.59_{-0.05}^{+0.05}$ & $0.57_{-0.05}^{+0.05}$ & $-0.03_{-0.09}^{+0.10}$ & $1.80_{-0.21}^{+0.24}$ \\
\hline $122 \ldots \ldots$ & $002403.8-720621$ & 0.95 & 0.94 & $381.4_{-19.5}^{+22.1}$ & $76.7_{-8.7}^{+10.2}$ & $219.1_{-14.8}^{+16.2}$ & $85.7_{-9.2}^{+11.0}$ & $-0.44_{-0.05}^{+0.06}$ & $0.48_{-0.05}^{+0.06}$ & $0.06_{-0.08}^{+0.09}$ & $1.59_{-0.18}^{-0.18}$ \\
\hline $117 \ldots \ldots$ & $002413.7-720302$ & 0.95 & 0.94 & $342.7_{-18.4}^{+21.0}$ & $74.9_{-8.6}^{+10.1}$ & $198.4_{-14.1}^{+15.5}$ & $69.3_{-8.3}^{+10.0}$ & $-0.48_{-0.06}^{+0.06}$ & $0.45_{-0.06}^{+0.06}$ & $-0.04_{-0.09}^{+0.09}$ & $1.62_{-0.20}^{+0.20}$ \\
\hline $44 \ldots \ldots \ldots$ & $002403.6-720459$ & 0.86 & 1.00 & $338.8_{-18.3}^{+21.4}$ & $81.5_{-90}^{+10.5}$ & $168.7_{-13.0}^{+14.1}$ & $88.6_{-9.4}^{+11.1}$ & $-0.31_{-0.06}^{+0.00}$ & $0.35_{-0.06}^{+0.06}$ & $0.04_{-0.08}^{+0.08}$ & $1.41_{-0.17}^{+0.17}$ \\
\hline
\end{tabular}

Notes.-Additional properties of the 47 Tuc X-ray sources from the 2002 data set are given. See $\S 2.3$ for details. Table 5 is published in its entirety in the electronic edition of the Astrophysical Journal. A portion is shown here for guidance regarding its form and content.

Finally, we computed background-subtracted photometry for each source in several bands. We calculate counts and fluxes for bands used in the globular cluster literature, $0.5-1.5,1.5-6$, $0.5-4.5,0.5-2.5$, and $0.5-6.0 \mathrm{keV}$ (see G01a; Grindlay et al. 2001b; Pooley et al. 2002a, 2002b; Heinke et al. 2003a). We also computed the fluxes for several other bands for use in making specialized color-color diagrams; these bands are $0.3-$ $0.8,0.8-2.0$, and $2.0-8.0 \mathrm{keV}$.

Below we explain the columns in Tables 2, 3, and 5. All errors are $1 \sigma$, containing $68 \%$ confidence for one parameter of interest.

Column (1): Chandra wavdetect detection number, following the detection and naming convention systems of G01a, Grindlay et al. (2002), and Edmonds et al. (2003a, 2003b) for sources detected in the 2000 data set (up to W184) and adding new numbers above W184 for new detections.

Column (2): IAU-approved source name.

Columns (3), (4), and (5): Position, corrected to the MSP frame; errors in each coordinate, expressed in seconds (not arcseconds) for right ascension; and distance from the center of the cluster (taken to be $\alpha=00^{\mathrm{h}} 24^{\mathrm{m}} 05^{\mathrm{s}} .29, \delta=-72^{\circ} 04^{\prime} 52^{\prime \prime} .3$; de Marchi et al. 1996).

Columns (6), (7), and (8): Background-subtracted counts in three standard bands.
Columns (9) and (10): X-ray luminosities computed for 0.56.0 and $0.5-2.5 \mathrm{keV}$ bands. Photon fluxes were computed from the recorded counts, effective apertures, exposure times, and effective area functions for each source. Assuming a $2 \mathrm{keV}$ VMEKAL spectrum (a reasonable approximation for the average spectrum of the fainter X-ray sources; see $\S \S 5$ and 7), an absorbing column of $1.3 \times 10^{20} \mathrm{~cm}^{-2}$, and a distance of $4.85 \mathrm{kpc}$, we derive conversion factors of $5.93 \times 10^{36}$ and $4.94 \times 10^{36} \mathrm{ergs}$ photon ${ }^{-1} \mathrm{~cm}^{2}$ to convert from photon flux to unabsorbed X-ray luminosity in the $0.5-6.0$ and $0.5-2.5 \mathrm{keV}$ energy bands, respectively. To recover the directly measured photon fluxes in photons $\mathrm{cm}^{-2} \mathrm{~s}^{-1}$, the reader may divide the quoted luminosities by these factors.

Columns (11), (12), and (13): Notes on individual sources: ID type (note that a question mark indicates a tentative classification; other IDs are secure classifications; qLX indicates a qLMXB, AGB indicates an AB from A01 identified with an $\mathrm{X}$-ray source in this work), other names (AKO 9 from Auriere et al. 1989; ROSAT X number from Verbunt \& Hasinger 1998; MSP lettering from Camilo et al. 2000; Freire et al. 2001; Edmonds et al. 2002a), and notes on variability ( Y, D, and H for $99.9 \%$ confidence variability on timescales of years, days, and hours, respectively; D? and $\mathrm{H}$ ? for $99 \%$ confidence variability on day or hour timescales). A " $c$ " indicates that the source may 
suffer from confusion. An " $\mathrm{m}$ " indicates that the source was identified manually, rather than by wavdetect.

Table 3 is the same as Table 2, except that we include the PSF fraction (col. [5]) and fractional exposure time (col. [6], compared to $72,155 \mathrm{~s}$ ) instead of the radial distance. Notes D and $\mathrm{H}$ indicate variability seen on day or hour timescales within the 2000 data set. In addition, the notes identify (with a " $\mathrm{t}$ ") the three sources detected by wavdetect in 2000 that are not detected in the 2002 data; two of these may be spurious detections.

The columns for Table 5 are as follows:

Column (3): Fraction of the $1.5 \mathrm{keV}$ PSF enclosed by our extraction region for each source.

Column (4): Fractional exposure time for each source, compared to the nominal exposure time of 281,074 s.

Columns (5), (6), (7), and (8): Counts in four supplementary bands.

Columns (9), (10), (11), and (12): Hardness ratios. HR1, HR2, and HR3 are defined as $(H-S) /(H+S)$, and HR4 is defined as $S / H$. For HR1, $H=2.0-8.0 \mathrm{keV}, S=0.8-2.0 \mathrm{keV}$; for HR2, $H=0.8-2.0, S=0.3-0.8 \mathrm{keV}$; for HR3, $H=$ $2.0-8.0 \mathrm{keV}, S=0.3-0.8 \mathrm{keV}$; for HR4, $H=1.5-6.0 \mathrm{keV}$, $S=0.5-1.5 \mathrm{keV}$.

\section{VARIABILITY ANALYSIS}

We here attempt to quantify the variability of our sources on a variety of timescales: during individual ObsIDs (hours), between ObsIDs (days), between the 2000 and 2002 observations (years), and (for the brightest sources) between the 2002 observations and the ROSAT observations of the 1990s discussed in Verbunt \& Hasinger (1998).

For intra-ObsID and inter-ObsID variability searches, we exclude the shortest exposures, which can independently detect only a few sources, leaving two exposures in the 2000 data and four in the 2002 data. For the 2002 data we use the $0.3-6.0 \mathrm{keV}$ events, while for the 2000 data we use the $0.5-6.0 \mathrm{keV}$ events. To identify variability on a timescale of hours, we applied a Kolmogorov-Smirnov test to the unbinned arrival times of the events from each observation. ${ }^{18}$ Sources that exhibited shortterm variability at the $99.9 \%$ confidence level in at least one observation are marked $\mathrm{H}$ for short-term "hours" variability in Tables 2 and 3, while sources that showed variability at the $99 \%$ level are marked $\mathrm{H}$ ? for possibly short-term variable. In this way we identify two short-term variables (six including possible variables) in the 2000 data set and 25 short-term variables (51 including possible variables) in the 2002 data set. Considering the 146 sources in the 2000 data set (and two searched exposures) as 292 trials, three of the possibly variable sources may not be variable. Using the four exposures and 300 sources in the 2002 data, 12 of the possibly variable sources may not be variable. These estimates, based on the K-S probabilities, may overestimate the amount of spurious variability, since the faintest sources considered do not provide sufficient counts in a single exposure for meaningful use of the K-S test (Press et al. 1992). All six variable or possibly variable sources in the 2000 data set show high-confidence short-term variability in the 2002 data set.

To identify longer term (timescale of days) variability, we compare the count rates between long exposures of a source (with the same instrumental setup), ignoring the effect of back-

${ }^{18}$ We used ACIS_EXTRACT ver. 3.5 to compute K-S probabilities. ground (which is very low in these observations). We define a significance level $\sigma$,

$$
\sigma=\frac{\left|\left[C_{j}-(\mathrm{ER}) C_{i}\right]\right|}{\sqrt{C_{j}+(\mathrm{ER})^{2} C_{i}}},
$$

where the exposure ratio ER is the exposure time times the effective area for observation $j$ divided by the exposure time and effective area for observation $i$, and $C_{j}$ and $C_{i}$ are the counts recorded from the source in each observation (Feigelson et al. 2002). We identify sources showing $\sigma>2.6$ as possibly variable (D?; 99\% confidence) and sources showing $\sigma>3.3$ as variable (D; $99.9 \%$ confidence) between exposures. Four sources show high-confidence days timescale variability between the two 2000 observations, while 31 sources show days timescale variability (53 including possible days timescale variability) among the 2002 observations. Considering the six possible combinations of 2002 observations, 18 of the possibly variable sources may not be variable, but again this could be an overestimate (see above).

Variability is more complicated to measure between observations using different instruments. This is because the change in effective areas at different energies may cause a source, with a spectrum different from that which is assumed, to appear to be variable even if it is not. To reduce this problem (and allow comparison with ROSAT results for some sources), we compare fluxes in the $0.5-2.5 \mathrm{keV}$ band, rather than the broader $0.5-6 \mathrm{keV}$ band, and only identify sources with $99.9 \%$ certainty of variability. We compute $\chi^{2}$ as $\chi^{2}=\left(F_{2002}-F_{2000}\right)^{2} /\left(\sigma_{2002}^{2}+\sigma_{2000}^{2}\right)$, using the upper or lower errors appropriately, and identify variability if $\chi^{2}>10.827$. These sources are indicated with a $Y$, for variability on timescales of years, in Tables 2, 3, and 5 . Three sources in the 2000 data are not seen in the 2002 data set, including W68 and two other possibly spurious sources. Some sources in the 2002 data would have been detected in the 2000 data had their emission remained constant, but they are not detected in the 2000 data. To identify these sources, we use equation (2) from Muno et al. (2003) to estimate the upper limits (at a signal-to-noise ratio of 3 ) at the locations of 2002 sources not detected in the 2000 data. We use background areas encircling $90 \%$ of the $1.5 \mathrm{keV}$ energy, with radii approximated (for our limited range of off-axis angles) by $r=2.65-0.14 \theta+$ $0.18 \theta^{2}$, with $r$ in arcseconds and $\theta$ in arcminutes. Sources with flux in 2002 more than $3 \sigma$ above these upper limits are identified as clearly variable; we find five such sources.

We perform the same tests for the nine X-ray sources identified by Verbunt \& Hasinger (1998) in the ROSAT data, taken between 1992 April and 1996 November. For these sources we recompute the ROSAT X-ray luminosities using the counts of Verbunt \& Hasinger (1998), a $2 \mathrm{keV}$ thermal plasma model, and PIMMS. ${ }^{19}$ We approximate the upper limit of detectability to be the flux from X19 (the dimmest source within the core region) for other sources within the core. For sources outside the core we use the flux from X13 as an upper limit. In all cases we identify variability at the $99.9 \%$ level. The results of these tests are listed in Table 6, along with the conclusions from Verbunt \& Hasinger (1998) about variability within the ROSAT data set.

Ascertaining variability is of particular interest because some groups of X-ray sources in 47 Tuc are expected to show intrinsic variability, while others are not. MSPs are not expected to show variability (on the timescales we can detect, using $3.2 \mathrm{~s}$ resolution

19 Available at http://asc.harvard.edu/toolkit/pimms.jsp. 
TABLE 6

ROSAT Flux Estimates and Variability

\begin{tabular}{|c|c|c|c|c|c|}
\hline \multicolumn{2}{|c|}{ Chandra NAMES } & \multirow[b]{2}{*}{$R O S A T$ NAME } & \multirow[b]{2}{*}{ Class } & \multirow{2}{*}{$\begin{array}{c}\text { ROSAT } L_{\mathrm{X}} \\
\left(10^{30} \mathrm{ergs} \mathrm{s}^{-1}\right)\end{array}$} & \multirow[b]{2}{*}{ VARIABLE? } \\
\hline W & CXOGlb J & & & & \\
\hline 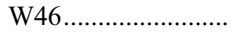 & $002403.4-720451$ & $\mathrm{X} 7$ & qLX & $1450.4 \pm 80.6$ & \\
\hline W42 ......................... & $002404.2-720458$ & $\mathrm{X} 9$ & $\mathrm{CV}$ & $919.7 \pm 68.7$ & $\mathrm{~T}, \mathrm{~V}, \mathrm{D}, \mathrm{H}, \mathrm{Y}$ \\
\hline 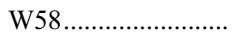 & $002400.9-720453$ & $\mathrm{X} 5$ & qLX & $1076.8 \pm 56.8$ & $\mathrm{~T}, \mathrm{D}, \mathrm{H}, \mathrm{Y}$ \\
\hline 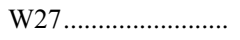 & $002406.3-720443$ & $\mathrm{X} 10$ & $\mathrm{CV}$ & $236.1 \pm 25.1$ & $\mathrm{~T}, \mathrm{~V}, \mathrm{D}, \mathrm{H}$ \\
\hline 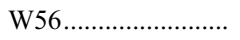 & $002402.1-720542$ & $\mathrm{X} 6$ & $\mathrm{CV}$ & $77.9 \pm 11.9$ & $\mathrm{D}, \mathrm{H}, \mathrm{Y}$ \\
\hline $\mathrm{W} 1$ & $002416.9-720427$ & $\ldots$ & $\mathrm{CV}$ & $<31.7$ & $\mathrm{~T}, \mathrm{H}$ ?, Y \\
\hline 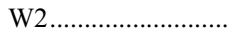 & $002415.8-720436$ & $\mathrm{X} 13$ & $\mathrm{CV}$ & $31.7 \pm 7.9$ & $\mathrm{D}, \mathrm{H}, \mathrm{Y}$ \\
\hline W125 ........................ & $002353.9-720350$ & $\mathrm{X} 4$ & qLX? & $64.7 \pm 11.9$ & $\mathrm{D} ?, \mathrm{H}$ \\
\hline 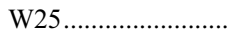 & $002407.1-720545$ & $\mathrm{X} 11$ & $\mathrm{CV}$ & $51.5 \pm 10.6$ & $\mathrm{D}$ \\
\hline W30 ................................ & $002406.0-720456$ & X19 & $\mathrm{CV}$ & $153.1 \pm 19.8$ & $\mathrm{~T}, \mathrm{D}, \mathrm{Y}$ \\
\hline
\end{tabular}

Notes.-X-ray sources detected by ROSAT in 47 Tuc, and one X-ray source detected by Chandra (W1), which would have been detected by ROSAT if it had been at its 2002 flux level in the $1990 \mathrm{~s}$. ROSAT $0.5-2.5 \mathrm{keV} \mathrm{X-ray} \mathrm{luminosities} \mathrm{are} \mathrm{derived}$ from the source counts of Verbunt \& Hasinger (1998) assuming a $2 \mathrm{keV}$ Raymond-Smith thermal plasma spectrum with the cluster absorption column and a distance of $4.85 \mathrm{kpc}$. T indicates sources that varied between the 1990s and 2002 (timescale of tens of years), while V indicates sources that varied during the 1990s (Verbunt \& Hasinger 1998). D, H, and Y indicate variability within the 2002 observations, or between the 2000 and 2002 observations (see Table 2).

times), and qLMXBs may not show intrinsic variability if their $\mathrm{X}$-ray emission is generated by release of heat from their cores (Brown et al. 1998), although they may vary owing to eclipsing behavior or variations in $N_{\mathrm{H}}$ column (Heinke et al. 2003b). On the other hand, $\mathrm{CVs}$ and $\mathrm{ABs}$ are expected to show a variety of variable behaviors, including flickering and flares (due to magnetic reconnection events in ABs). The hypothesis that most $\mathrm{ABs}$ in the X-ray luminosity range probed by these observations would appear as transient sources, flaring to detectability for a few hours and then dropping below our detection limit, is proven incorrect. Most ABs in our data are detectable in all observations of equal depth, and many show no evidence of variability even when $>100$ counts are detected.

The MSP 47 Tuc $\mathrm{O}$ is identified as long-term variable $(3.7 \sigma)$ between the second and fourth 2002 observations, appearing to decrease continuously in flux from the second to the fourth observation. The MSP 47 Tuc $U$ is also possibly variable within the third 2002 observation, and 47 Tuc R is possibly variable between observations in 2002. No physical mechanism has been proposed, to our knowledge, that would explain significant variability of the flux from these old MSPs on these timescales. We think it most likely that W39, only a few arcseconds from the core of the cluster, is a blend of 47 Tuc $\mathrm{O}$ and another, variable $\mathrm{X}$-ray source, most likely an AB (see S. Bogdanov et al. 2005, in preparation and Fig. 2). A portion of our sources are certain to be blends, and we have marked some likely confused sources in Table 2. The MSP 47 Tuc R is only 1".3 from the brighter, variable source W24, which may cause a spurious detection of variability. For 47 Tuc $\mathrm{U}$ we can say only that there is a reasonable probability that one MSP will be spuriously identified as possibly variable. The X-ray sources W6, W31, W71, W91, W96, and W97, identified as potential MSPs by Edmonds et al. (2003b), show variability or possible variability and thus should be considered less likely MSP candidates. However, three of these (W6, W91, W96) are only classified as possibly variable.

We note that all sources with more than 1000 counts (13) are identified as variable, except for the qLMXB X7, the brightest source in the field (>30,000 counts), which shows no indication of variability within the 2002 data. This indicates that the mechanism driving the $\mathrm{X}$-ray emission is probably different from that in Aquila X-1 and Cen X-4, where significant variability on short timescales is generally thought to be due to accretion (Rutledge et al. 2002; Campana et al. 2004; Heinke et al. 2003b). X10 displays very strong periodic variability $(P=16,804 \mathrm{~s})$, with a phased light curve (Fig. 5) similar to those seen in the magnetic CVs known as polars (Ramsay et al. 2004). The AB W47 and the CV W51 each show spectacular flaring behavior in the 2002 data set, reaching an energy output of several times $10^{33} \mathrm{ergs} \mathrm{s}^{-1}$ at the peak (Fig. 6). Searches for possible periodicities, long-term variability between the 2000 and 2002 Chandra observations (and ROSAT observations), and more detailed characterization of timing results will be presented in future papers (J. E. Grindlay et al. 2005, in preparation).

\section{RADIAL DISTRIBUTIONS}

In order to understand how many of the sources we have detected are background sources versus cluster members, we study the 2002 detected sources' radial distributions. (The 2000 data are significantly incomplete owing to chip gaps, so we disregard them in this section.) The effective area of Chandra's mirrors does not decrease significantly within our 2'.79 (47 Tuc

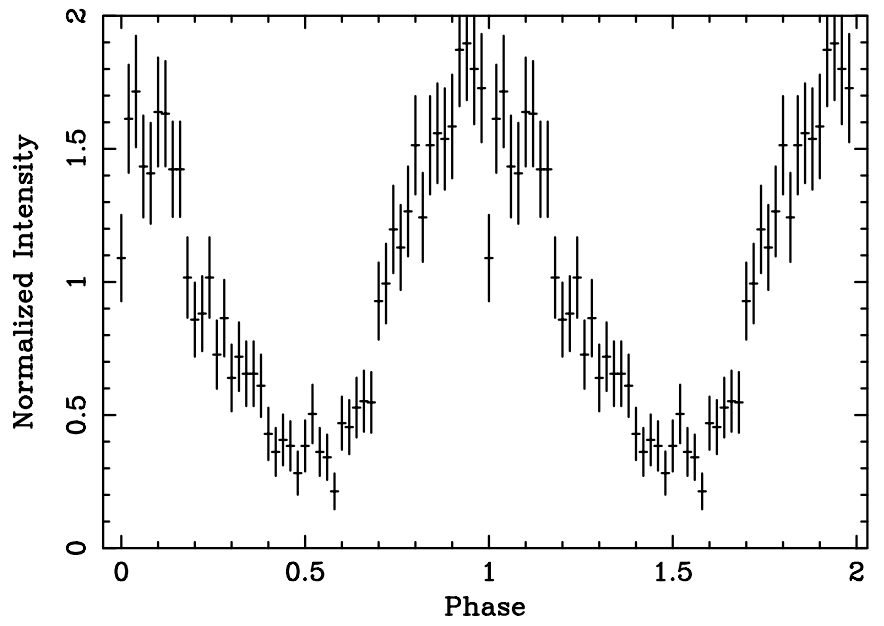

FIG. 5.-Folded X-ray light curve for the CV X10 (W27) on a 16,804 s period. Two cycles are shown for clarity. 


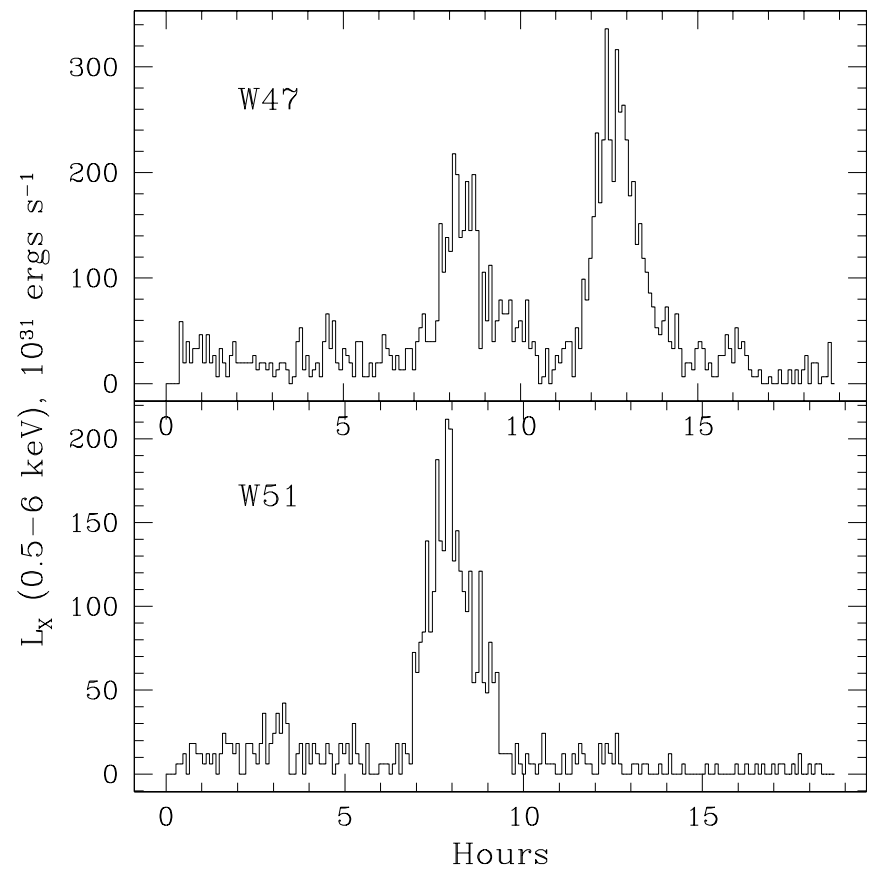

FIG. 6.-Background-subtracted light curves of two sources showing extraordinary flares. Conversions from count rates to X-ray luminosities are calculated using the time-averaged spectra, which we have confirmed are reasonable spectral representations of the flares. These conversions are $2.2 \times 10^{34}$ ergs count $^{-1}$ for W47 (top; ObsID 2737), an AB, and $2.0 \times 10^{34} \mathrm{ergs} \mathrm{count}^{-1}$ for W51 (bottom; ObsID 2735), a CV. Each became briefly as luminous as any other source in the cluster.

half-mass radius) survey area (effective area at 2.'79 is $\sim 98 \%$ of on-axis value for $1.5 \mathrm{keV}$ photons; Chandra Proposer's Observatory Guide), and our covered area lies almost entirely $(99 \%)$ on the ACIS-S3 chip. Thus, our sensitivity does not change dramatically across our surveyed area. In Figure 7 we show the distribution of $0.3-8 \mathrm{keV}$ counts versus distance from the cluster center, both in core radii $\left(24^{\prime \prime}\right)$ and in units of square arcminutes, where background sources should be evenly spread. Figure 7 and careful examination of the data indicate that essentially all sources above 20 counts $(0.3-8 \mathrm{keV})$ have been detected (although in many cases we detect much weaker sources).

This is not strictly true where sources lie close enough together to overlap; the existence of 75 sources above 20 counts within the core of 47 Tuc produces a $3.5 \%$ chance of one of these sources landing within 0.5 of another source. This suggests that two or three sources above our detection limit within 47 Tuc's core are unresolvably confused with other sources (since the pixel scale of the ACIS detector is only 0"492). A further approximately six to seven should be located within $1^{\prime \prime}$ of other sources, which may be recognized as confused sources inconsistent with the Chandra PSF. Many of these should be separable using more complicated detection algorithms, such as two-dimensional K-S tests (Metchev \& Grindlay 2002); this work is in progress. An example of two sources that are confused in our source detection scheme is 47 Tuc S and 47 Tuc F, two radio MSPs located 0"74 from each other and detected as the single, clearly confused source W77. An additional two to three faint sources may also be lost within $2^{\prime \prime}-3^{\prime \prime}$ of the three brightest sources in 47 Tuc, X7, X9, and X5, where the PSF wings are capable of obscuring sources at our detection limit. Thus, we probably miss some 12 sources from inside the core radius. We bear in mind these caveats in the analysis that follows.
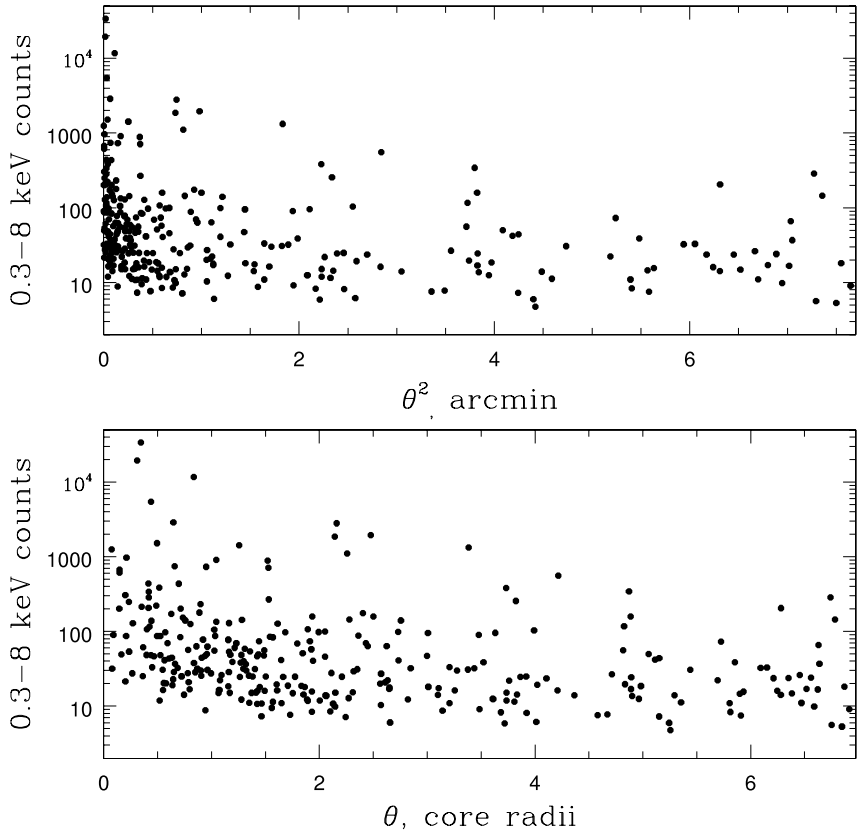

FIG. 7.-Distributions of extracted source counts vs. radial distance from the center of 47 Tuc (de Marchi et al. 1996). Top: Radial distance plotted in units of $\operatorname{arcmin}^{2}$, so background sources should be evenly distributed. Bottom: Radial distance plotted in units of 47 Tuc core radii $\left(1 r_{c}=24^{\prime \prime}\right)$. Beyond about $100^{\prime \prime}$ $\left(2.77 \operatorname{arcmin}^{2}, 4.17 r_{c}\right)$, the source density appears flat.

We first estimate the background density. It can be seen directly from Figure 7 that the number of sources per square arcminute appears flat beyond roughly $100^{\prime \prime}\left(2.78 \operatorname{arcmin}^{2}\right)$. A background level of $1.5 \pm 0.3$ sources arcminute ${ }^{-2}$ (error derived from Poisson statistics) gives an asymptotically flat cumulative excess of sources above the background beyond $\sim 100^{\prime \prime}$, for a total of 37 background sources within the half-mass radius. Therefore, the 24 sources with $>20$ net counts that lie between $100^{\prime \prime}$ and the 47 Tuc half-mass radius are consistent with being background sources.

We estimate the expected background extragalactic source numbers from the cumulative number counts $(0.5-2.0 \mathrm{keV}$ band, where we detect nearly all of our sources) of Brandt et al. (2001),

$$
N(>S)=3970\left(S / 10^{-16} \operatorname{ergs~} \mathrm{cm}^{-2} \mathrm{~s}^{-1}\right)^{-0.67 \pm 0.14} \mathrm{deg}^{-2}
$$

which was derived from sources at the flux levels to which we are sensitive. Assuming a $\Gamma=1.4$ photon index power-law spectrum (Giacconi et al. 2001; Brandt et al. 2001) and $N_{\mathrm{H}}=$ $1.3 \times 10^{20} \mathrm{~cm}^{-2}, 20$ counts is a $0.5-2 \mathrm{keV}$ flux of $1.63 \times$ $10^{-16} \mathrm{ergs} \mathrm{cm}^{-2} \mathrm{~s}^{-1}$, giving $2860 \pm 200$ sources $\mathrm{deg}^{-2}$. Thus, we should find $19 \pm 4$ background active galactic nuclei (AGNs) above 20 counts within the half-mass radius. This is roughly half of the number of sources we detect, which suggests an enhancement over the deep-field number counts of Brandt et al. (2001). This level of enhancement is unlikely to be due to cosmic variance in extragalactic number counts (Kim et al. 2004). Below 20 counts, uncertainties on our flux measurements due to noise and confusion may cause a preferential shift of our fluxes to higher values (a version of Eddington bias; Murdoch et al. 1973; Muno et al. 2003), but this should not be a large problem above 20 counts since the slope of our overall luminosity function is relatively flat (see $\S 6$ ). This enhancement could be 
caused by background X-ray sources in the nearby Small Magellanic Cloud. Alternatively, these X-ray sources may reside in 47 Tuc's halo, either because they are relatively low mass binaries pushed out by mass segregation, or because they were generated by primordial binaries in the distant halo (Davies 1997; Gendre et al. 2003). Some close binaries are known to exist in 47 Tuc's halo (Kaluzny et al. 1998; Weldrake et al. 2004), and blue stragglers in 47 Tuc's halo are thought to be generated by primordial binaries (Ferraro et al. 2004). The nature of this X-ray background population will be probed in future papers that examine the full Chandra fields.

The radial distribution of objects in a dynamically relaxed cluster allows an estimate of the average mass of those objects, in terms of a mass ratio between the object and the average mass of the typical stars that define the central gravitational potential (Grindlay et al. 1984, 2002). Heinke et al. (2003c) describe a procedure for estimating the typical qLMXB mass from the spatial distribution of a sample of 20 probable qLMXBs in seven clusters. This procedure is based on maximum likelihood fitting of a parameterized form to the radial profile of the source distribution. The key parameter is the ratio $q=M_{\mathrm{X}} / M_{*}$ of the source mass to the mass of the typical stars that define the optical core radius. This approach assumes that the spatial distribution of these typical stars is well described by a classical King (1966) model, reasonable for 47 Tuc (Howell et al. 2000). Then the radial profile for the source density takes the form

$$
S(r)=S_{0}\left[1+\left(\frac{r}{r_{c *}}\right)^{2}\right]^{(1-3 q) / 2},
$$

where $S_{0}$ is an overall normalization and $r_{c^{*}}$ is the optical core radius determined for turnoff-mass stars (here we use $24^{\prime \prime}$ ). We correct the $>20$ count source sample for background contamination using the estimate of 1.5 sources $\operatorname{arcmin}^{-2}$ and the bootstrap resampling procedure described in Grindlay et al. (2002). The best-fitting value for $q=M_{\mathrm{X}} / M_{*}$ is $1.63 \pm 0.11$, consistent with the value of $q=1.5 \pm 0.25$ found for soft sources in Grindlay et al. (2002), and the cumulative radial distribution is compared with this model in Figure 8. Restricting our sample to sources with $>40$ counts, we find identical results, indicating that crowding in the core does not dramatically affect these results.

To estimate the mass of these sources requires an estimate of the mass of the core stars to which we are comparing our sources. The core radius we use is from Howell et al. (2000), which uses a limiting $U$ magnitude of 18.11 , or $V=17.6$. Using the model isochrones of Bergbusch \& VandenBerg (2001) as displayed in Briley et al. (2004), the mass range included in Howell et al. (2000) is from 0.865 to $0.915 M_{\odot}$. We take the average mass of the stars that determine the core radius of 47 Tuc to be $0.88 \pm$ $0.05 M_{\odot}$, allowing for some uncertainty in the modeling (e.g., in the distance to $47 \mathrm{Tuc}$ ). We also include the $8 \%$ uncertainty in the Howell et al. (2000) core radius determination. Our result for the X-ray sources is an average mass of $M_{\mathrm{X}}=1.43 \pm 0.17 M_{\odot}$ for the X-ray sources. This is consistent with our expectations for a mixture of qLMXBs, CVs, ABs, and MSPs, all of which are significantly heavier than single main-sequence stars.

We can also constrain the masses of individual populations within the GO-8267 HST field, incorporating the radial incompleteness of the WFPC2 field. Including all $60 \mathrm{ABs}$ and candidate ABs within this field produces $q=1.12 \pm 0.10$, implying a typical mass $M_{\mathrm{X}}=0.99 \pm 0.13 M_{\odot}$. This is significantly lower (at the $4.6 \sigma$ level) than the average $q$-value of the X-ray sources as a whole, calculated above. This does not

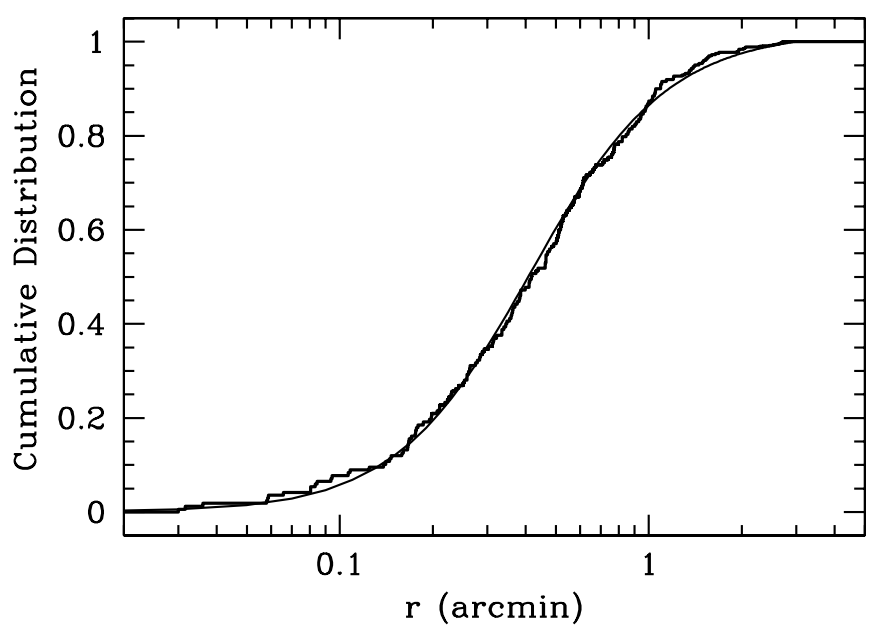

FIG. 8.-Plot of cumulative radial distribution of 47 Tuc X-ray sources (above 20 counts, $0.3-8 \mathrm{keV}$ energy band) fitted to an analytical model (eq. [2]) for their radial distribution. The best fit, plotted here, gives a ratio of average source mass to typical stellar mass of $q=1.63$.

take into account our reduced sensitivity to X-ray sources in the cluster core. Restricting our fits to the $43 \mathrm{ABs}$ with $>20$ counts gives $q=1.32 \pm 0.13$, only $2.4 \sigma$ from the mean X-ray source $q$. However, there is a correlation between X-ray flux and $\mathrm{AB}$ mass (Edmonds et al. 2003b), so that ABs brighter in X-rays may be expected to be more massive. Starting from the full sample of $60 \mathrm{ABs}$ and candidates within the GO-8267 field of view, we exclude an inner core of increasing size, from 0 to 1.5 core radii. The value of $q$ produced by each sample remains essentially the same (ranging from 1.10 to 1.14), leading us to conclude that the relatively low mass of ABs (compared to the average X-ray source) is robust.

We can perform the same test (with lesser significance) for the $\mathrm{CV}$ and MSP populations. For the $22 \mathrm{CVs}$ and $\mathrm{CV}$ candidates within the GO-8267 field, we derive $q=1.49 \pm 0.20$, inferring a mass of $M_{\mathrm{X}}=1.31 \pm 0.22 M_{\odot}$, consistent with our rough expectations for CVs containing heavy white dwarfs plus secondary stars. For the $22 \mathrm{MSPs}$ and MSP candidates (not including R) in the GO-8267 field of view, we find $q=1.42 \pm 0.16$, implying $M_{\mathrm{X}}=1.25 \pm 0.19 M_{\odot}$. This group seems less massive than expected, which suggests that several of the MSP candidates identified as possible MSPs by Edmonds et al. (2003b) are not actually MSPs (as suspected by Edmonds et al. 2003b).

For the 17 securely identified MSPs (not including R) with previously known positions (radially complete to $6^{\prime}$; Camilo et al. 2000), we derive $q=1.67 \pm 0.14$, implying $M_{\mathrm{X}}=1.47 \pm$ $0.19 M_{\odot}$. We can compare this number with the expected average mass of the known MSP binary systems in 47 Tuc, using the total mass of 10 binary companions $\left(1.33 \pm 0.11 M_{\odot}\right.$, using a mean value of $1 / \sin i$ of $4 / \pi ;$ Chandrasekhar \& Münch 1950; Backer 1998) to find an average neutron star mass of $1.39 \pm$ $0.19 M_{\odot}$. Comparing this with the average pulsar mass of $1.35 \pm 0.05 M_{\odot}$ (Thorsett \& Chakrabarty 1999), we see that MSPs in 47 Tuc have not accreted a substantial amount of mass (less than $0.23 M_{\odot}$ ) during their recycling (in agreement with Thorsett \& Chakrabarty 1999).

\section{PHOTOMETRY}

We produced an X-ray version of a CMD for the 2002 data (Fig. 9), plotting broadband luminosity versus the hardness ratio $2.5 \log [(0.5-1.5 \mathrm{keV}$ counts $) /(1.5-6 \mathrm{keV}$ counts) $]$ (G01a; Grindlay et al. 2001b; Pooley et al. 2002a, 2002b). We found the 


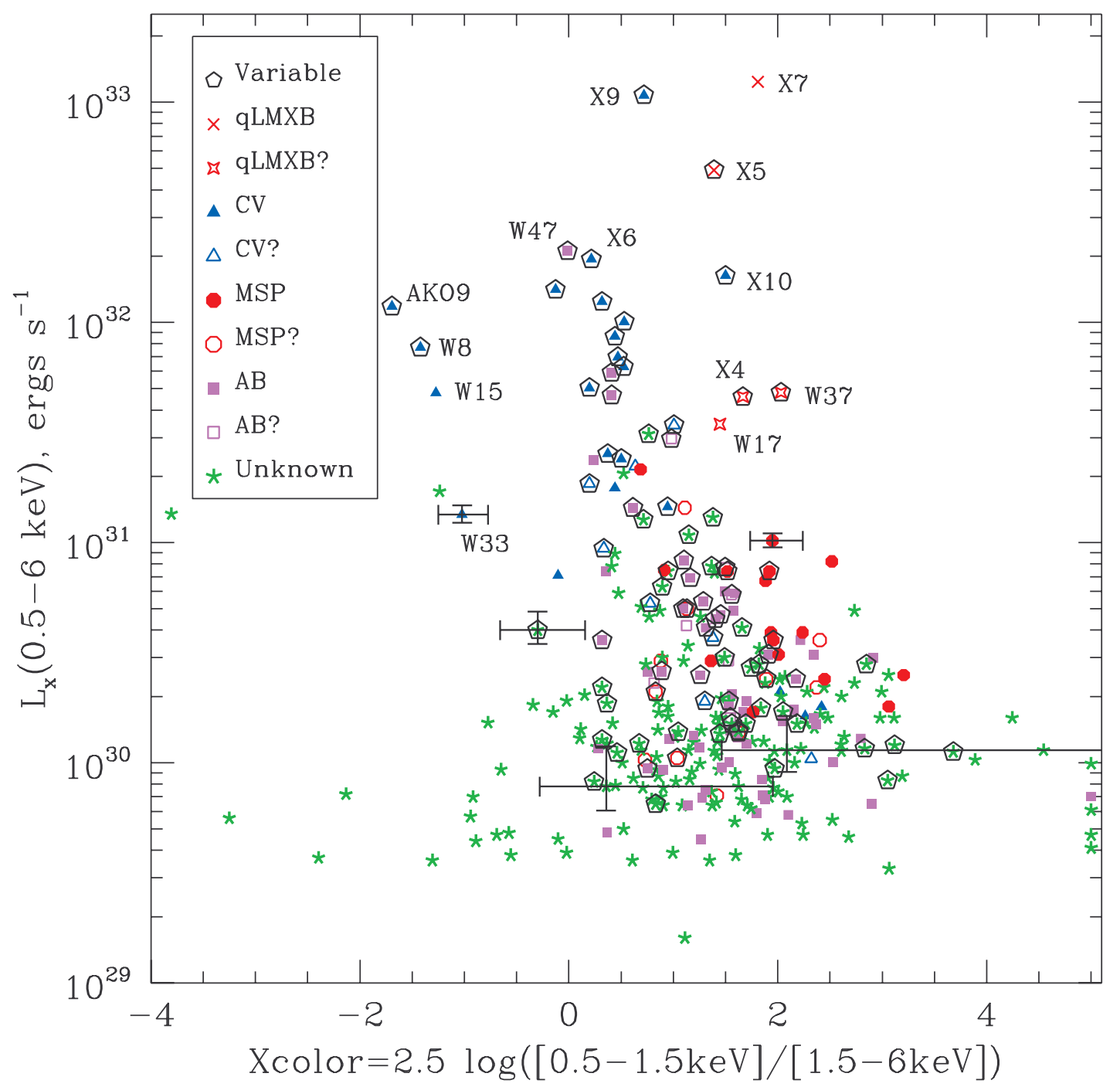

FIG. 9.-X-ray CMD, plotting X-ray luminosity (0.5-6 keV, absorbed) against hardness (increasing to the left) for 47 Tuc X-ray sources from 2002 data. X-ray luminosities are taken from spectral fits to thermal plasmas (Table 7, col. [7]) or, for fainter sources, from the photon fluxes using a conversion assuming a $2 \mathrm{keV}$ VMEKAL spectrum (Table 2). The variable sources ( $>99 \%$ confidence) are indicated with pentagons. Only a few error bars are plotted, to improve readability. Several sources of particular interest are indicated with their most common names.

most useful quantity for the $y$-axis to be the observed $0.5-6 \mathrm{keV}$ flux, which we took from spectral fits to a VMEKAL model (§ 7) when sufficient counts were available for simple spectral fits. (We use the observed $0.5-6 \mathrm{keV}$ flux, not correcting for absorption, since in many cases the $N_{\mathrm{H}}$ column is poorly determined.) Otherwise, we used the X-ray luminosities computed using a $2 \mathrm{keV}$ VMEKAL model (see $\S 7$ ) and the photon fluxes computed above $(\S 2.3$ ). (The actual luminosities will be dependent on the intrinsic spectral shape. We choose a $2 \mathrm{keV}$ VMEKAL model as a reasonable average for these spectra, since the hardness ratios are poorly constrained for these faint sources.) We have indicated all the major classifications of identified sources and whether each source shows variability above the $99 \%$ confidence level within the 2002 data or shows variability between the 2000 and 2002 data sets. This plot is qualitatively similar to the X-ray CMD shown in G01a for the 2000 data, but our choice of $y$-axes shifts the harder sources up on the diagram.

We have plotted this X-ray CMD again in Figure 10, indicating X-ray sources located at distances beyond $100^{\prime \prime}$ by placing squares around the symbols (all but one of unknown type). These sources appear to be mostly background sources, and many are likely AGNs. At least $64 \%$ of the background objects in the figure should lie beyond $100^{\prime \prime}$. We have also plotted the positions of various spectral models, with the known cluster absorption unless otherwise indicated. The equivalent hardness ratios from power-law spectra are indicated at the bottom, for several representative photon indices. Hydrogen atmosphere neutron star models are plotted for a range of temperatures (in $\mathrm{eV}$ ), assuming a $10 \mathrm{~km}$ radius (adding a contribution from a power-law spectral component would harden these spectra). Thermal plasma models (VMEKAL in XSPEC), using 47 Tuc elemental abundances, are plotted for a constant volume emission measure (VEM) of $2 \times 10^{55} \mathrm{~cm}^{-3}$, for a range of temperatures in $\mathrm{keV}$ (indicated). The effect of increasing $N_{\mathrm{H}}$ on a $10 \mathrm{keV}$ thermal plasma spectrum (with a lower, arbitrary VEM) is indicated, for several values of $N_{\mathrm{H}}$.

It can be clearly seen that most $\mathrm{CVs}$ and $\mathrm{ABs}$ follow the same trends in these diagrams. Above $L_{X}(0.5-6 \mathrm{keV})=10^{31} \mathrm{ergs}$ $\mathrm{s}^{-1}$, most are consistent with thermal plasma of $5-10+\mathrm{keV}$. Below $L_{\mathrm{X}}=10^{31} \mathrm{ergs} \mathrm{s}^{-1}$, their temperatures tend to decrease, but with increasing scatter (the scatter in the sources is significantly larger than the scatter due to counting statistics). For 


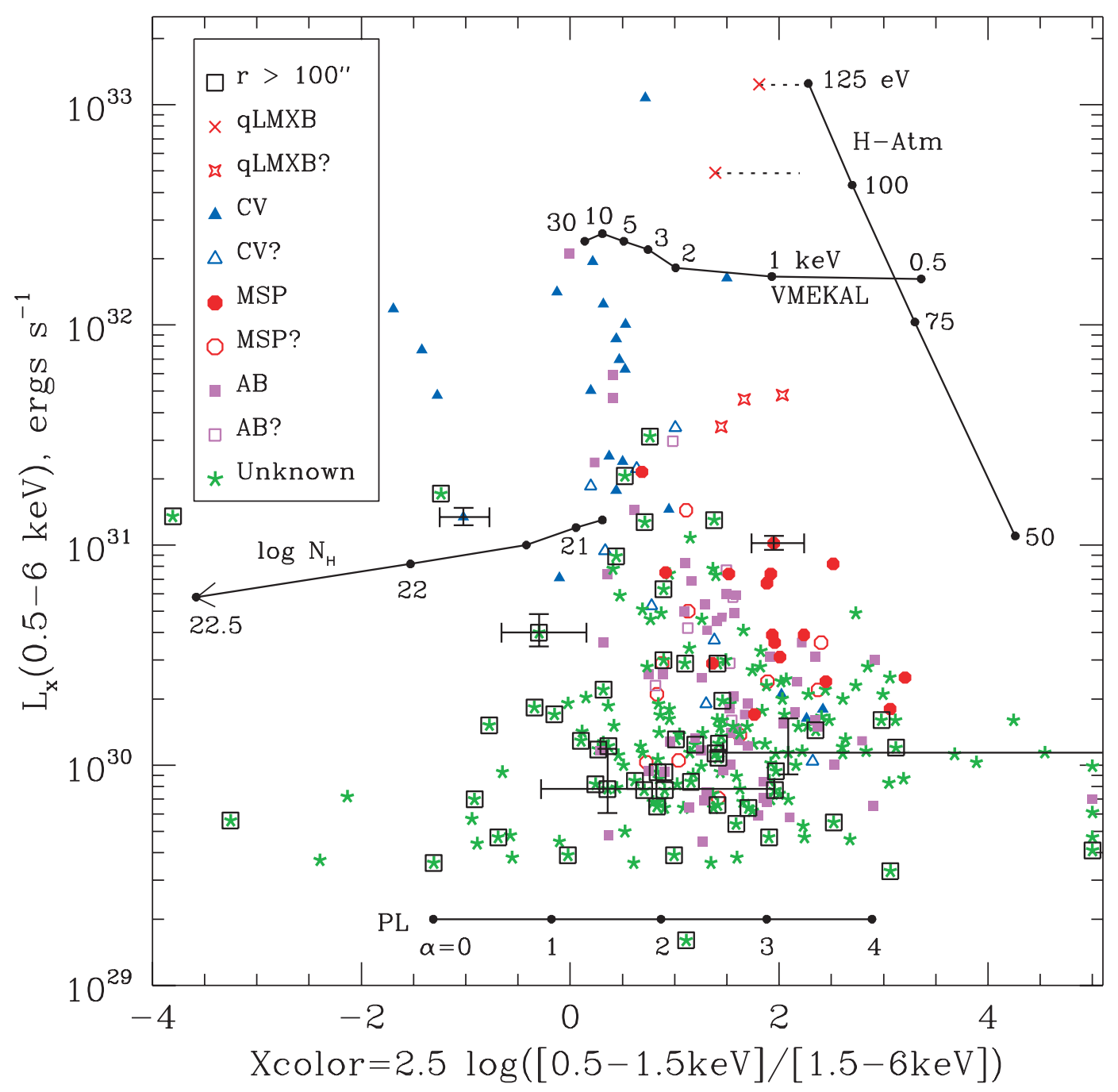

FIG. 10.-X-ray CMD, plotting X-ray luminosity (0.5-6 keV) against hardness (increasing to the left) for 47 Tuc X-ray sources from 2002 data, as in Fig. 9. Sources located beyond $100^{\prime \prime}$ are indicated by squares and are largely background. The location of several model spectra are also indicated, assuming the cluster $N_{\mathrm{H}}$ column except where indicated. The spectra are marked with units of temperature (for $\mathrm{H}$ atmosphere in $\mathrm{eV}$ and VMEKAL in $\mathrm{keV}$ ), photon index (for the power law), and $N_{\mathrm{H}}$ in $\log \mathrm{cm}^{-2}$. The vertical locations of the VMEKAL, power-law, and $N_{\mathrm{H}}$ lines are arbitrary, while the effect of increasing $N_{\mathrm{H}}$ (shown here for a $10 \mathrm{keV}$ VMEKAL spectrum) varies depending on the input spectrum. Dotted lines next to the qLMXBs X5 and X7 indicate the shift in their X colors when only the subarray data (with mitigated pileup) are used.

their hardness ratios, MSPs appear to be slightly brighter on average than most other source classes. Several sources are significantly harder than a thermal plasma spectrum can become without added absorption. Three of these sources are the known eclipsing CVs AKO 9, W8, and W15 (G01a; Edmonds et al. 2003a; Knigge et al. 2003). The fainter hard sources are predominantly located beyond $100^{\prime \prime}$ from the cluster, indicating that many are background AGNs with intrinsic absorption (as suggested by Grindlay et al. 2002).

Several bright soft sources can be identified, which do not follow our expectations. The bright and extremely soft CV X10 is discussed in $\S \S 3$ and 7 and is a likely magnetic CV showing a soft excess at its accreting magnetic pole(s). Few sources fall near the canonical $\mathrm{H}$ atmosphere qLMXB track, which is well populated in some other globular clusters (Heinke et al. 2003c). For the known qLMXB X7 this can be explained by the effects of photon pileup in the detector artificially hardening the spectrum. For the eclipsing qLMXB X5, this is due to a large and highly variable gas column, as well as pileup hardening. We have indicated (dotted lines) the shifts in X-ray color for the known qLMXBs X7 and X5, by computing their X-ray color during subarray exposures, where the effects of pileup are decreased (for X5, we use only ObsID 3385, in which X5 is brightest). We also identify three unusual, moderately bright yet soft sources (W37, X4 or W125, and W17). These sources could be qLMXBs if they have very large gas columns or significant nonthermal spectral components. We address these sources further in $\S 7$ and in Heinke et al. (2005).

We constructed color-color plots, using different hardness ratios of the form $(H-S) /(H+S)$, with the bands 2.0$8.0 \mathrm{keV}=H, 0.8-2.0 \mathrm{keV}=S$ (HR1), 0.8-2.0 keV $=H, 0.3-$ $0.8 \mathrm{keV}=S$ (HR2), and $2.0-8.0 \mathrm{keV}=H, 0.3-0.8 \mathrm{keV}=S$ (HR3). We select these bands in order to maximize the differences between a thermal plasma model and emission in the form of a power law. At low temperatures thermal plasma (even at the low metallicity of 47 Tuc) will show a relatively larger flux in the $0.8-2.0 \mathrm{keV}$ band owing to Fe L-shell line emission (see Fig. 11, showing the spectra of a typical faint $\mathrm{AB}$ and an MSP). Since we expect the spectra of faint, soft ABs and CVs to be generated by hot optically thin plasma, this allows some differentiation between them and MSPs, which should not show Fe L-shell line emission. 

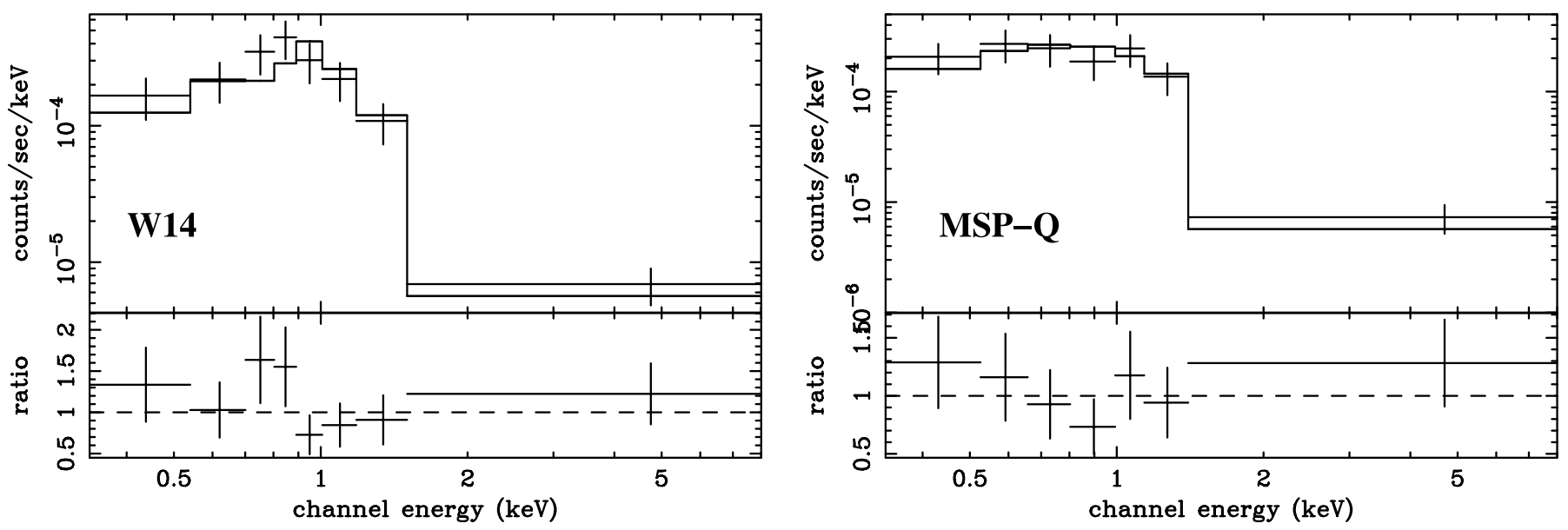

FIg. 11.-Energy spectra of the AB W14 (left) and the MSP 47 Tuc Q (W104; right). W14 is fitted with a thermal plasma spectrum with temperature $1.0 \pm 0.5 \mathrm{keV}$ and $N_{\mathrm{H}}=1.3 \times 10^{20} \mathrm{~cm}^{-2}$ and shows prominent Fe L emission lines (included in this fit). The MSP 47 Tuc Q is poorly fitted by a thermal plasma spectrum and is here fitted by a $137 \mathrm{eV}$ hydrogen atmosphere model spectrum (details in S. Bogdanov et al. 2005, in preparation).

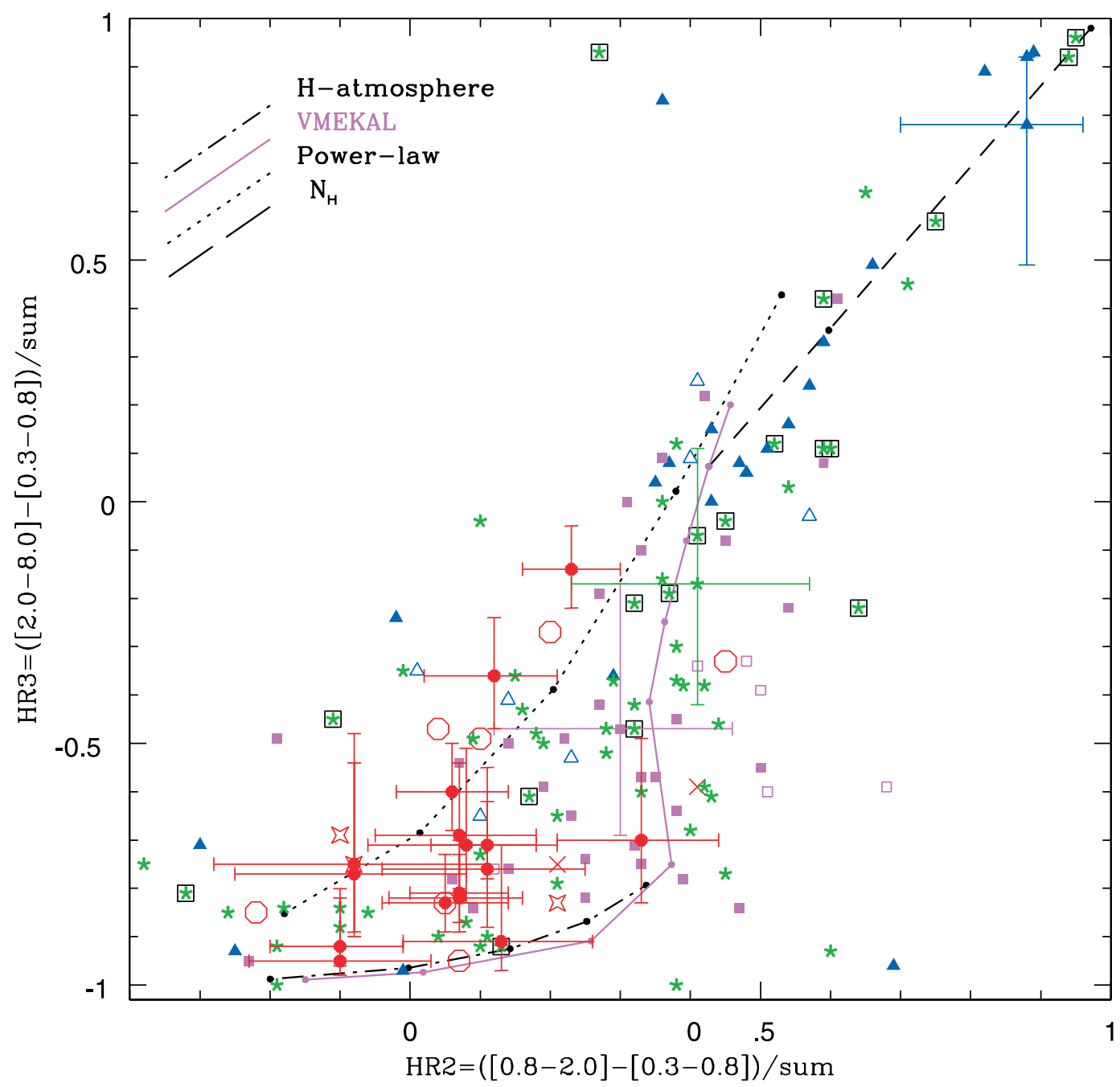

FIG. 12.-Color-color diagram for 47 Tuc sources with more than 30 counts. Symbols as in Fig. 10. Error bars are plotted for all MSPs and a few representative faint sources. Model spectra tracks are plotted, with dots representing the following values from lower left to upper right: $\mathrm{H}$ atmosphere for $75,100,125,150$, and $175 \mathrm{eV}$; VMEKAL thermal plasma model (see text) for $0.4,0.5,0.7,1,2,3,5,10$, and $30 \mathrm{keV}$; power law, photon index $\alpha=3,2.5,2,1.5,1$; and effect of increasing $N_{\mathrm{H}}$ on a $10 \mathrm{keV}$ VMEKAL spectrum for $1.3 \times 10^{20}$ (standard), $10^{21}$, and $10^{22} \mathrm{~cm}^{-2}$. 


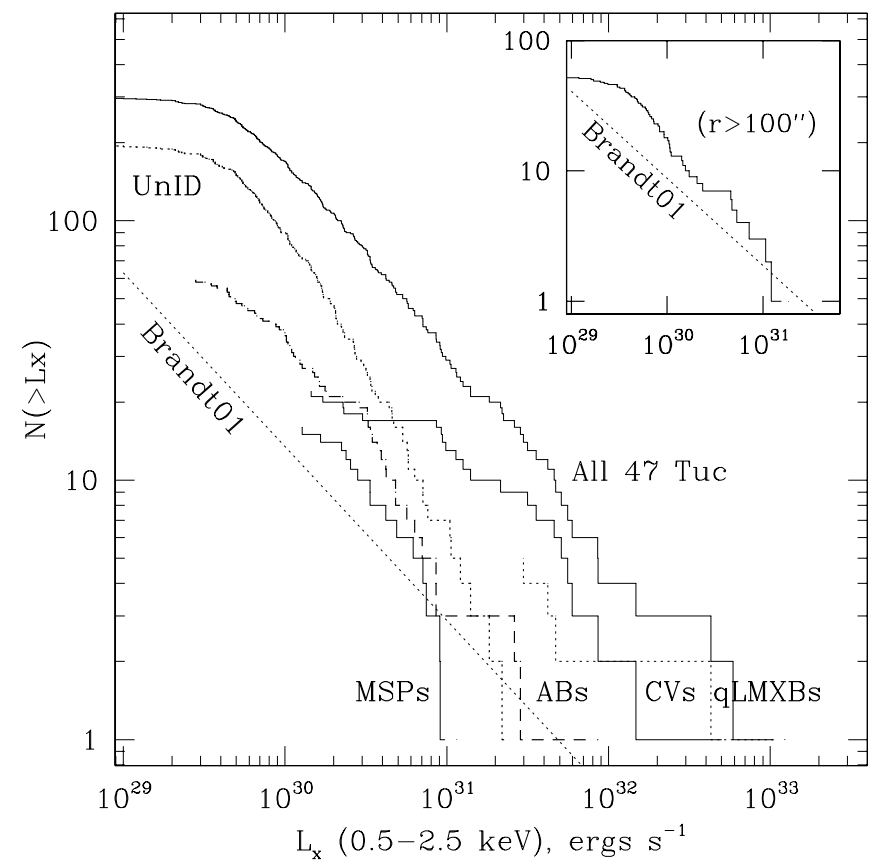

FIG. 13.-Cumulative luminosity functions for the different classes of identified sources in 47 Tuc: CVs, ABs, qLMXBs (including W37, W17, and $\mathrm{X} 4$ /W125), and MSPs. Luminosity functions are also plotted for the total source population, the unknown sources (including candidate ABs, MSPs, and CVs), and the predicted extragalactic source counts from Brandt et al. (2001). We begin to be incomplete below 20 counts, roughly $8 \times 10^{29} \operatorname{ergs~s}^{-1}$. The inset shows sources beyond $100^{\prime \prime}$, which have a radial distribution consistent with being background but exceed the Brandt et al. (2001) prediction (shown for that area). [See the electronic edition of the Journal for a color version of this figure.]

In Figure 12 we plot HR2 against HR3 for the subset of sources with more than 30 total counts (including all known MSPs). We exclude faint sources in order to decrease the scatter. Source symbols are the same as in Figure 10. Errors are indicated for all MSPs and a few other relatively faint representative sources. A clear difference can be seen in the distributions of ABs and CVs in the diagram versus the distribution of MSPs. We have overplotted the locations of three spectral models for a range of parameters: a thermal plasma model with 47 Tuc abundances (VMEKAL), a hydrogen atmosphere neutron star model (since the normalization is not relevant, this is appropriate for the surface of either MSPs or qLMXBs), and a power law with specified photon indices. We have also indicated the effect of increasing $N_{\mathrm{H}}$ for a $10 \mathrm{keV}$ thermal plasma model.

It is clear from Figure 12 that a thermal plasma model, with temperatures generally between 0.7 and $10 \mathrm{keV}$, is a reasonable description of the colors of most of the ABs and CVs (some with increased $N_{\mathrm{H}}$ ) and a large number of the unknown sources. The MSPs, on the other hand, lie between the tracks for hydrogen atmosphere neutron star models and power-law models. Grindlay et al. (2002) used a similar color-color method on the 2000 data alone to indicate that the MSPs in 47 Tuc were dominated by thermal emission. Here we see that a two-component spectrum is likely to best explain the overall data, including a hydrogen atmosphere model between 75 and $175 \mathrm{eV}$ and a powerlaw component with photon index between 3 and 1.5. The MSP spectra and colors are analyzed in detail in S. Bogdanov et al. (2005, in preparation).

It appears that the unknown sources have X-ray colors similar to the identified sources. We attempt to constrain the relative fractions of the unidentified sources by comparison with the colors of known sources in $\S 8$.

\section{LUMINOSITY FUNCTIONS}

We construct luminosity functions for the X-ray sources seen in the 2002 data using the $0.5-2.5 \mathrm{keV}$ fluxes from Table 2 . We show the cumulative luminosity functions of each source class in Figure 13 and the differential number counts in Figure 14. We also plot the expected background counts, using the extragalactic source density from Brandt et al. (2001). Our putative completeness limit is $L_{\mathrm{X}}(0.5-2.5 \mathrm{keV})=8 \times 10^{29} \mathrm{ergs} \mathrm{s}^{-1}$, although many sources are detected below this limit. The inset of Figure 13 illustrates the luminosity function of the apparent background sources, which is substantially above the expected extragalactic counts. Significant cosmic variance is not seen in extragalactic Chandra source counts (Kim et al. 2004), so another population of objects is indicated, which may be halo sources in 47 Tuc (Davies 1997). If so, the resemblance of their luminosity function to ABs suggests that they are primordial short-period $\mathrm{ABs}$ and represent the progenitor population to the halo blue stragglers (Ferraro et al. 2004). Figure 14 also shows the sources in each luminosity bin that we identify as variable or possibly variable.

The forms of the MSP and qLMXB differential luminosity functions do not appear to resemble power laws. The MSPs were not X-ray selected, and qLMXBs have not been observed at luminosities below those of our faintest candidate qLMXBs. We describe the luminosity functions of these two groups with lognormal distributions rather than power laws, while we fit the total source numbers, background sources, $\mathrm{CVs}$, and $\mathrm{ABs}$ with power-law distributions, using the maximum likelihood formalism of Crawford et al. (1970). To avoid Eddington bias strongly affecting the low ends of our luminosity functions, we fit the luminosity functions only for sources with $\gtrsim 25$ counts, or $10^{30} \mathrm{ergs} \mathrm{s}^{-1}$ for our data (Murdoch et al. 1973).

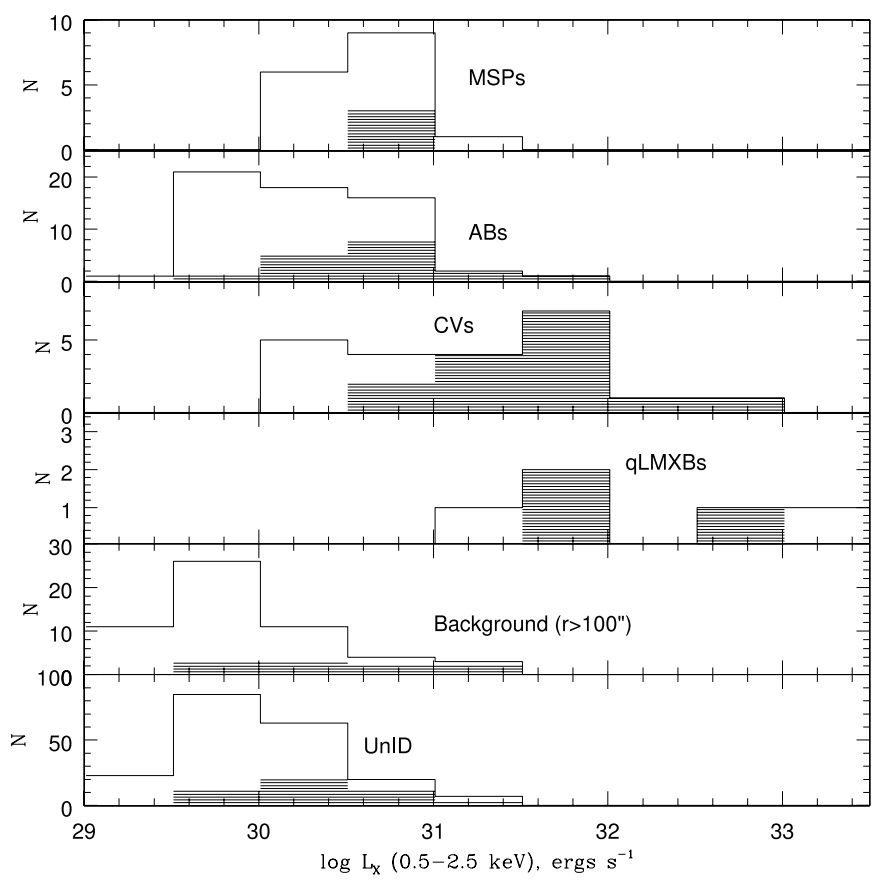

FIG. 14.-Differential number counts for the different classes of identified sources in 47 Tuc: CVs, ABs, MSPs, and qLMXBs (including W37, W17, and W125), as well as putative background sources and unknown sources (not including sources beyond $100^{\prime \prime}$ ). The shaded histograms indicate those sources that were detected to be variable at $>99 \%$ confidence (see text). Our completeness limit is $\sim 8 \times 10^{29} \mathrm{ergs} \mathrm{s}^{-1}$, but over half the sources are detected below that limit. 
TABLE 7

Spectral Fits to 47 Tuc Sources

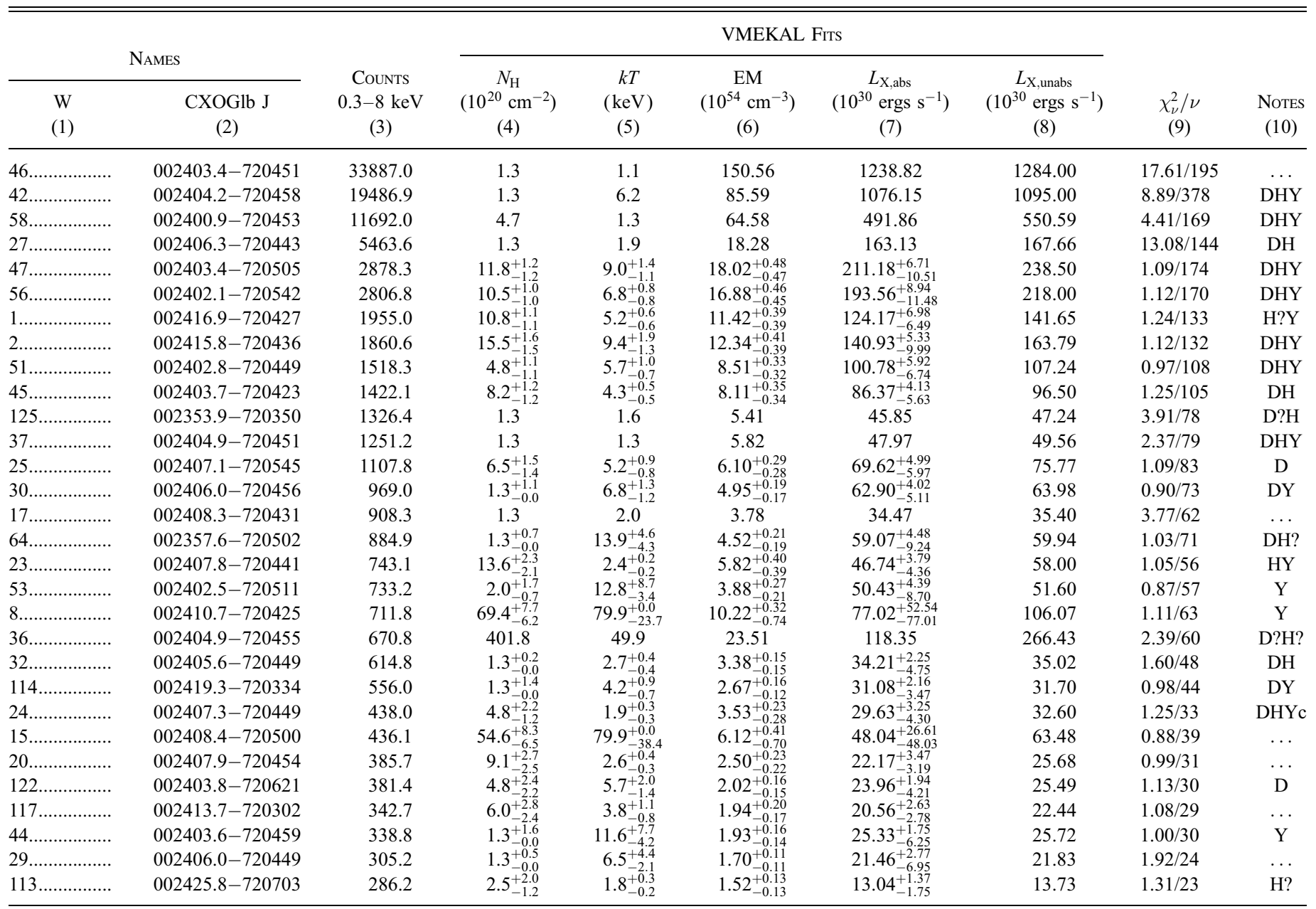

Notes.-Spectral fits to the 47 Tuc X-ray sources from the 2002 observations are given. Spectral fits use an absorbed thermal plasma VMEKAL model, with abundances set to those of 47 Tuc (see $\S 7$ ). When $\chi_{\nu}^{2}>2$, no errors are calculated for spectral parameters; otherwise, $1 \sigma$ errors are quoted. $L_{\mathrm{X} \text {,abs }}$ is the inferred $0.5-6 \mathrm{keV}$ X-ray luminosity without accounting for absorption, and $L_{\mathrm{X}, \text { unabs }}$ is the deabsorbed $0.5-6 \mathrm{keV}$ X-ray luminosity implied by the fitted $N_{\mathrm{H}}$ (and should be treated with skepticism). Notes indicate variability or confusion, which may affect the spectral parameters: H and D indicate hours and days timescale variability at $99.9 \%$ confidence, $\mathrm{H}$ ? and D? indicate possible hours and days timescale variability at $99 \%$ confidence, and $\mathrm{Y}$ indicates years timescale variability. A "c" indicates a possibly confused source, while an " $\mathrm{m}$ " indicates a source added manually. Table 7 is published in its entirety in the electronic edition of the Astrophysical Journal. A portion is shown here for guidance regarding its form and content.

For the total source population of 47 Tuc, we find a good power-law fit, $N(>S) \propto S^{-\alpha}$, with $\alpha=0.71 \pm 0.05$, consistent with the fit reported by Pooley et al. (2002b), $\alpha=0.78_{-0.17}^{+0.16}$. For the $\mathrm{AB}$ population, our fit gives $\alpha=0.88 \pm 0.14$. We note that the median X-ray luminosity for BY Draconis systems in the field is $1.6 \times 10^{29} \mathrm{ergs} \mathrm{s}^{-1}$ (Dempsey et al. 1997), so we anticipate many more $\mathrm{ABs}$ in 47 Tuc below our detection limit. For the background sources, the calculated index is $0.94 \pm 0.22$. This is significantly steeper than the extragalactic luminosity function of Brandt et al. (2001; see eq. [1]), as can be seen in Figure 13.

The CV luminosities can be described by a power law with $\alpha=0.31 \pm 0.04$. The CVs can also be described by a lognormal distribution with mean $\log L_{\mathrm{X}}(0.5-2.5 \mathrm{keV})=31.2$ and standard deviation $\sigma_{\log L_{X}, \mathrm{CV}}=0.67$. This is substantially brighter than the average X-ray luminosities of Galactic CVs, as discussed in Edmonds et al. (2003b). However, since the known CVs are identified only from the (relatively bright) X-ray sources in G01a, we do not yet know the true distribution of CV X-ray luminosities in 47 Tuc.
The (five) qLMXB (and candidate) X-ray luminosities have a mean $\log L_{\mathrm{X}}(0.5-2.5 \mathrm{keV})=32.1$ and standard deviation $\sigma_{\log L_{X}, \mathrm{qLMXB}}=0.7$. The MSP luminosities have a mean $\log L_{X}(0.5-2.5 \mathrm{keV})=30.6$ and standard deviation $\sigma_{\log L_{X}, \mathrm{MSP}}=$ 0.28 (derived without the unresolved pairs $\mathrm{F} / \mathrm{S}$ and $\mathrm{G} / \mathrm{I}$, which are consistent with this distribution). MSPs in the field often have rather uncertain distances. This may be the best test of the luminosity distribution of old MSPs, since the distance and reddening to these MSPs are well known.

\section{SPECTRAL ANALYSIS}

We modeled the spectra of sources with more than $\sim 30$ counts between 0.35 and $8.0 \mathrm{keV}$ (147 sources), using the ACIS EXTRACT automated spectral fitting script (Broos et al. 2002) and XSPEC version 11.3. We binned the data using at least 10 counts bin ${ }^{-1}$ and used $\chi^{2}$ statistics. We tried several spectral models, including power-law, blackbody, bremsstrahlung, and one-temperature MEKAL continuum models (Mewe 1991) modified by the PHABS photoelectric absorption model. In all cases we constrained the hydrogen column to $\geq 1.3 \times 10^{20} \mathrm{~cm}^{-2}$, 
TABLE 8

Spectral Fits to 47 Tuc Sources, 2000 Observations

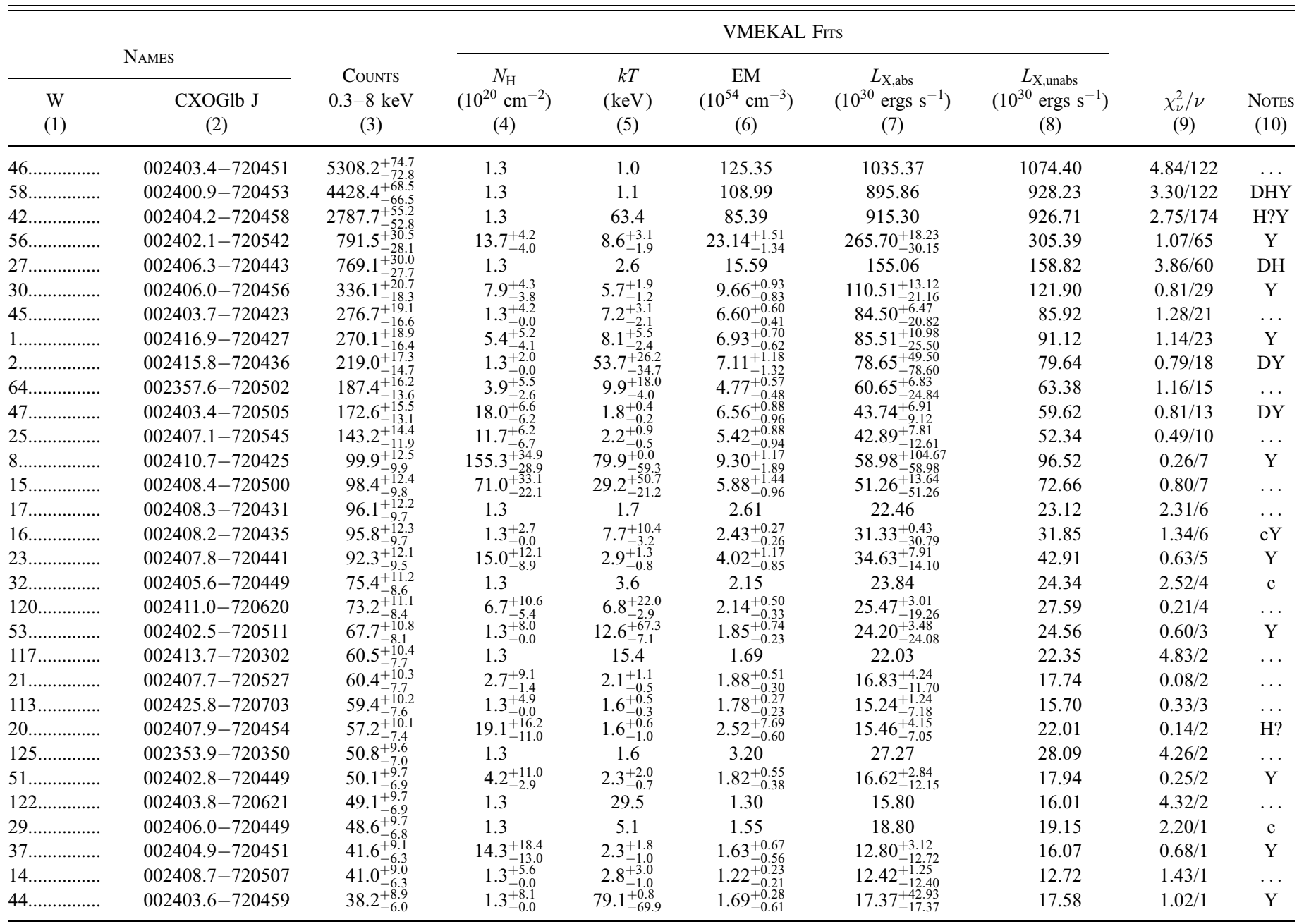

Notes.- Spectral fits to 47 Tuc X-ray sources from the 2000 observations are given. Only 31 sources are bright enough for spectral modeling, and all of these fits are presented here. Spectral fits use an absorbed thermal plasma VMEKAL model, with abundances set to those of 47 Tuc (see $\S 7$ ). Columns as in Table 7.

the known cluster value (Gratton et al. 2003). The model expected to best physically describe CVs and chromospherically active binaries is a thermal plasma model (comprising a bremsstrahlung continuum plus emission lines), which we characterize by a variable-abundances MEKAL model (VMEKAL in XSPEC). We fix the abundances of this model to appropriate values for 47 Tuc: $[\mathrm{Fe} / \mathrm{H}]=-0.70(20 \%$ solar $),[\mathrm{O} / \mathrm{Fe}]=0.50$ (60\% solar), and $[\mathrm{Si} / \mathrm{Fe}]=0.30$ (40\% solar) (Carney 1996; Salaris \& Weiss 1998). We assume that $\mathrm{C}, \mathrm{N}$, and $\mathrm{O}$ have similar abundances, Ne through $\mathrm{Ca}$ have similar abundances, and $\mathrm{Fe}$ and $\mathrm{Ni}$ have the same abundance. We present the results of our VMEKAL spectral fits to the 2002 data in Table 7 and to the 2000 data in Table 8. Detailed analyses of these spectra (including time-resolved spectra for variable sources) and timing properties of all sources will be the subject of additional papers. In the following we look at some general properties of the VMEKAL spectral fits to the 2002 data and briefly discuss some individual sources.

\subsection{Spectral Properties}

A total of 147 sources had sufficient counts for simple spectral fitting. Most of the spectra were reasonably well fitted (null hypothesis probability, or nhp, $>5 \%$ ) by the power-law (133/147 sources) and bremsstrahlung (129/147 sources) models, while the single-temperature VMEKAL model resulted in slightly fewer (105/147) good fits. However, the VMEKAL model returns values of $N_{\mathrm{H}}$ near the known cluster value for most ABs, unlike the power-law model. We anticipate that most ABs should not suffer enhanced extinction and choose the VMEKAL model as the most physical description for many of our sources. We show histograms of the derived temperatures for members of various source classes in Figure 15, along with the numbers of sources showing poor spectral fits. Multipletemperature plasmas may be required to understand some source spectra, while others are not expected to be well described by thermal plasmas at all (specifically MSPs and qLMXBs). We note that most (13/16) of the known MSPs, as well as all five qLMXBs or qLMXB candidates, are poorly fitted by thermal plasma models. One-third (7/21) of the fitted CVs are poorly fitted; this is partly due to their generally very high statistics (exposing calibration uncertainties) and partly due to complex models required to describe some of the brighter sources (see below). Other groups were better fitted: only 3/22 ABs, 1/7 candidate MSPs, 2/7 candidate CVs, none of the 15 sources beyond $100^{\prime \prime}$, and $11 / 40$ of the remaining unidentified sources were badly fitted.

We also plot the best-fit temperature against the $0.5-6 \mathrm{keV}$ $\mathrm{X}$-ray luminosity in Figure 16. We see a correlation between temperature and X-ray luminosity generally followed by both 


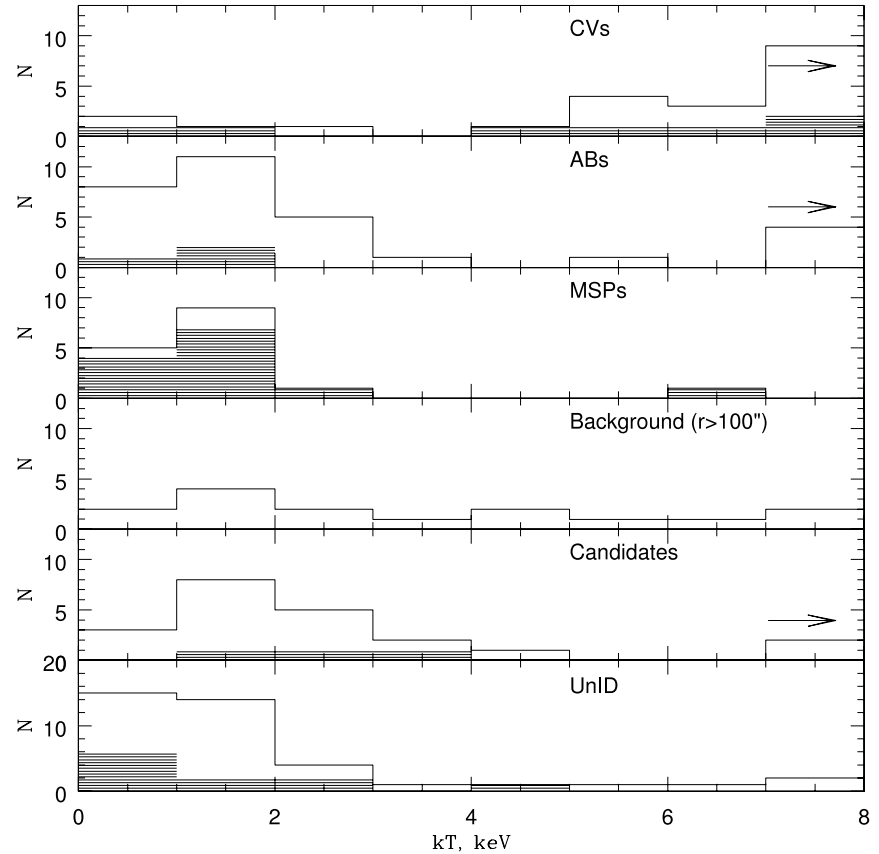

Fig. 15.-Histograms of fitted temperatures from thermal plasma model fits to five source classes. The group labeled "Candidates" includes possible counterparts indicated as CV?s, AB?s, and MSP?s in Edmonds et al. (2003b) and Table 2. All five qLMXBs and qLMXB candidates give poor fits and are not shown here. Bad fits producing null hypothesis probabilities $<5 \%$ are indicated by the shaded histograms. Any best-fit temperatures above $7 \mathrm{keV}$ (above which Chandra has little effective area) are included in the last bin.

ABs and CVs. For CVs we expect that both temperature and luminosity may increase with the white dwarf mass ( Wu et al. 1995; Cropper et al. 1998). No correlation of either temperature or luminosity with accretion rate has been found in previous studies (see van Teeseling et al. 1996). We note that there are several well-fitted CVs with temperatures that clearly lie below $10 \mathrm{keV}$. This may imply that their white dwarf masses lie below $0.4 M_{\odot}$ or that they have relatively high rates of cyclotron cooling ( Wu et al. 1995). The qLMXB candidates appear to have unusually low temperatures and high X-ray luminosities compared to other X-ray sources and are poorly fitted by plasma models (see also below).

Most of the X-ray sources are consistent with the cluster $N_{\mathrm{H}}$ value in the VMEKAL fits, but several are clearly not. We plot the derived $N_{\mathrm{H}}$ values from our thermal plasma fits in Figure 17, plotting errors only where the derived $N_{\mathrm{H}}$ is not consistent with the cluster value and where the fit produces $\chi_{\nu}^{2}<2.0$. Several categories of X-ray sources tend to show enhanced $N_{\mathrm{H}}$ : CVs, likely background sources, and candidate ABs. The likely background sources should contain extragalactic AGNs, which are often highly absorbed, so their $N_{\mathrm{H}}$ values are not surprising.

Twelve of 22 known $\mathrm{CVs}$ require intrinsic $N_{\mathrm{H}}$ columns. The known eclipsing CVs AKO 9, W8, and W15 (Edmonds et al. 2003b) clearly show very high $N_{\mathrm{H}}$, as expected for an edge-on inclination; W33 appears to have a similarly high $N_{\mathrm{H}}$ value, suggesting a similarly high inclination. The enhanced $N_{\mathrm{H}}$ values for most of the other bright CVs, near $10^{21} \mathrm{~cm}^{-2}$, indicate that we are seeing absorption from gas within these systems. Nonmagnetic systems at low inclination appear not to require enhanced $N_{\mathrm{H}}$ beyond that expected from interstellar material (Verbunt et al. 1997; Eracleous et al. 1991; van Teeseling et al. 1996). On the other hand, the moderately magnetic systems known as intermediate polars or DQ Hers often show increased

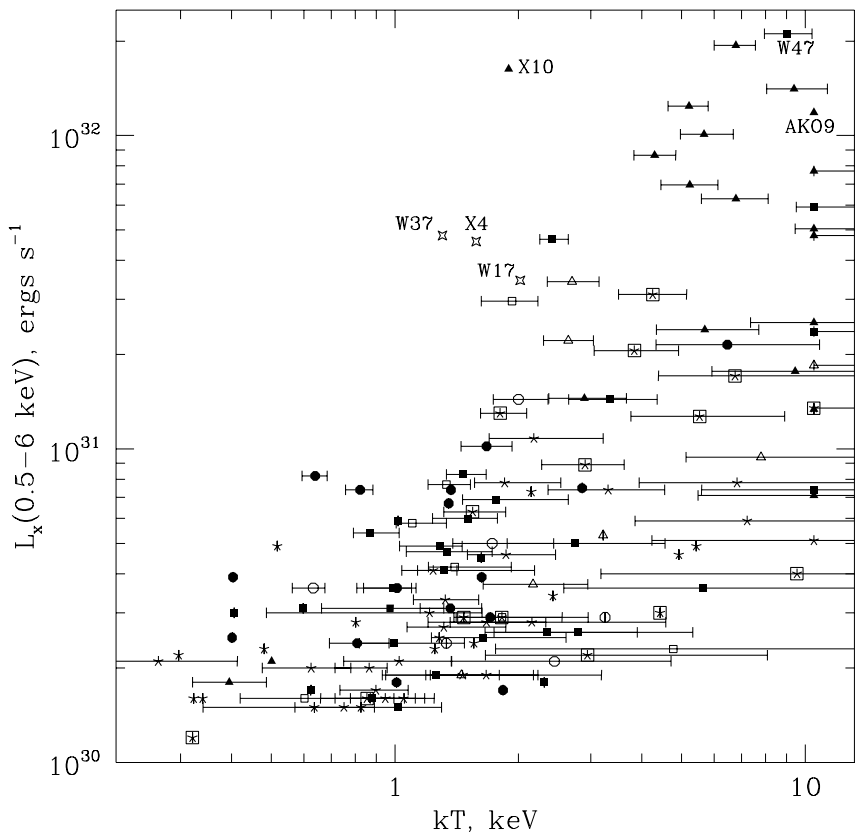

FIG. 16.-Parameter $k T$ from thermal plasma model fit, plotted against fitted $0.5-6 \mathrm{keV} L_{\mathrm{X}}$. Symbols as in Fig. 10 . Errors $(90 \%$ confidence) on $k T$ are not plotted for sources that are very badly fitted by our thermal plasma model $\left(\chi_{\nu}^{2} \geq 2.0\right)$, including the three relatively bright sources X7, X5, and X9 (left off this plot). Some unusual objects are marked. [See the electronic edition of the Journal for a color version of this figure.]

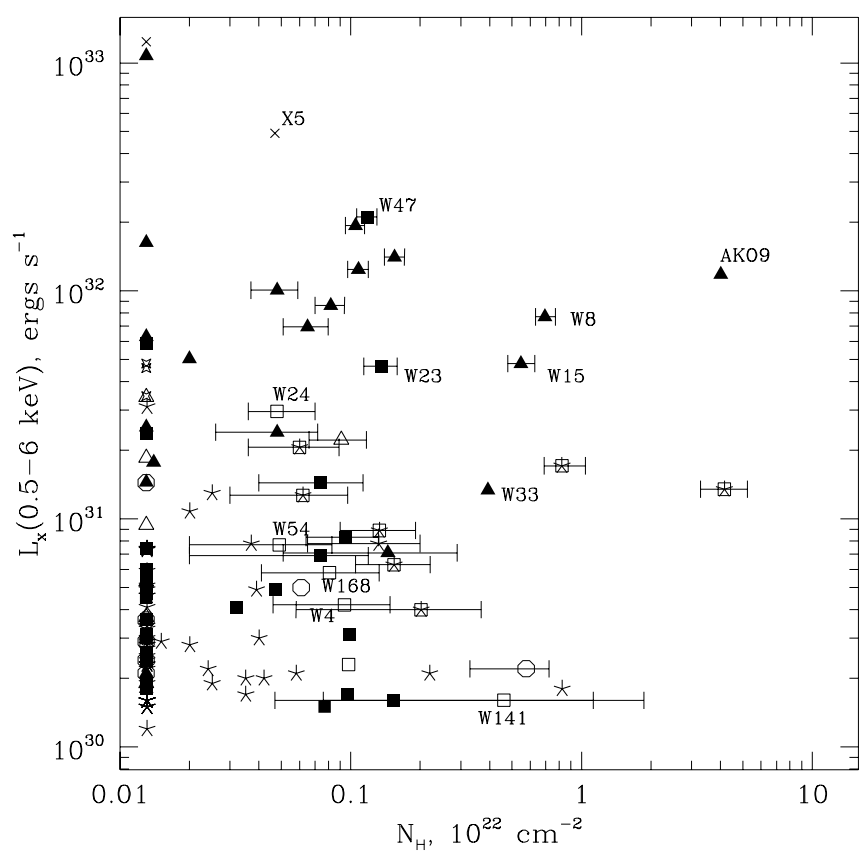

FIG. 17. $-N_{\mathrm{H}}$ from thermal plasma model fit, plotted against fitted $0.5-6 \mathrm{keV}$ $L_{\mathrm{X}}$. Symbols as in Fig. 10. Errors (90\% confidence) on $N_{\mathrm{H}}$ are plotted only for those sources that are well fitted by this model and that have absorption columns inconsistent with the cluster value, $N_{\mathrm{H}}=1.3 \times 10^{20} \mathrm{~cm}^{-2}$ (Gratton et al. 2003). Three objects that are not well fitted by the single thermal plasma model (and thus do not have plotted errors), but which clearly show extra absorption, are identified (X5, AKO 9, and W33). Four other sources that show unusually high absorption columns for their class are also identified, as are the five AB candidates from Edmonds et al. (2003b) with intrinsic absorption (see $\S$ 7.1). [See the electronic edition of the Journal for a color version of this figure.] 
$N_{\mathrm{H}}$ (Norton \& Watson 1989) and tend to have higher X-ray luminosities (although this may be affected by selection effects; Verbunt et al. 1997). It has already been suggested that X-raybright CVs in globular clusters may be preferentially magnetic systems (Grindlay et al. 1995; Edmonds et al. 1999, 2003b), and the enhanced $N_{\mathrm{H}}$ values tend to support this conclusion.

We are surprised by the intrinsic absorption seen in five of the six candidate ABs identified by Edmonds et al. (2003b), including W54. These systems were identified as possible RS CVn owing to their proximity to bright stars, subgiants, or giants, which were saturated in the GO-8267 HST data set. The discovery that their X-ray spectra are also special indicates that the bright stars are indeed related to these X-ray sources. (If the proximity of these X-ray sources to bright stars were due to coincidence, then these X-ray sources should not have a unifying X-ray characteristic.) We speculate that the enhanced $N_{\mathrm{H}}$ may be due to dense winds from the giant or subgiant stars. We have not, however, confirmed these stars to be RS CVn; other explanations, such as symbiotic stars (white dwarfs accreting from giant star winds), are still possible. These stars are likely to be amenable to ground-based optical photometry and spectroscopy, unlike the majority of X-ray counterparts in 47 Tuc.

The enhanced $N_{\mathrm{H}}$ from six ABs is also not understood. The brightest, W47, is a known semidetached W UMa binary, indicating that Roche lobe overflow is occurring and may be responsible for the occulting gas. Enhanced $N_{\mathrm{H}}$ has been observed for several chromospherically active stars (Favata \& Schmitt 1999; Maggio et al. 2000) at the level of $10^{19}-10^{20} \mathrm{~cm}^{-2}$ and has been attributed to coronal mass ejections. However, some of our ABs show $N_{\mathrm{H}} \sim 10^{21} \mathrm{~cm}^{-2}, 10$ times higher than observed in field systems.

\subsection{Individual Sources}

We briefly discuss a few sources that are poorly fitted by a thermal plasma model and have unusual values for their bestfitting parameters. The two bright qLMXBs X5 and X7 are very badly fitted, partly because of the effects of pileup on their very soft spectra, which we do not model here (Heinke et al. 2003b). (We note that the modeling of the hard spectra of the other relatively bright sources is less affected by pileup.) Hydrogen atmosphere modeling using the XSPEC pileup formalism provides excellent fits to these spectra; this fitting is discussed in A. Rybicki et al. (2005, in preparation). Most of the rest of the sources can be fitted by two-component spectra; we discuss the most interesting ones below.

\subsection{1. $C V s$}

The CV X9 (W42), the brightest non-qLMXB in the cluster, shows a peculiarly strong emission line at $0.65 \mathrm{keV}$, tentatively identified with $\mathrm{O}$ VIII, on a hard continuum. To model this spectrum requires two MEKAL components of similar emission measures, one at 0.25 and one at $>17 \mathrm{keV}$, with abundances set to those of the cluster. However, this does not result in a good fit, primarily because the spectrum contains so many counts $(20,000)$ that calibration residuals are clearly apparent. We note, in accord with Sanders et al. (2004), significant residuals between 1.3 and $2.3 \mathrm{keV}$ and an overestimate of the flux around $0.4 \mathrm{keV}$. We also see a significant underprediction of the flux above $6 \mathrm{keV}$, which cannot be accounted for by pileup, since it is also present in the spectrum generated from subarray observations. Some of this excess may be due to calibration errors. Most of the excess can be accounted for by adding a third plasma component, hidden behind a column of $(10-100) \times 10^{22} \mathrm{H} \mathrm{cm}^{-2}$. This would be consistent with partial covering models often invoked for the intermediate polar subset of CVs (Norton \& Watson 1989) and the possible detection of a $218 \mathrm{~s}$ period by G01a, which they suggested indicated a DQ Her classification. We show this model in Figure 18. In any case, X9's luminosity and apparent spectral hardness are similar to the Galactic center systems discussed by Muno et al. (2003, 2004).

The bright CV X10 (W27) exhibits a rather soft spectrum, poorly fitted by single-component models. It is the brightest source in the cluster in the poorly calibrated $0.1-0.3 \mathrm{keV}$ band, having 1240 counts in that band (compared to 732 for the bright, soft qLMXB X7), and we believe that correct understanding of its spectrum cannot be accomplished without including these soft X-rays. A spectral fit using two MEKAL components (at $0.39 \pm 0.03$ and $>14 \mathrm{keV}$ ), plus a blackbody with $k T=53 \pm 4 \mathrm{eV}$, provides a good fit above $0.5 \mathrm{keV}$ and a reasonable fit to most of the data below $0.5 \mathrm{keV}$ (Fig. 18). An apparently uncalibrated feature at $0.4 \mathrm{keV}$ is present in the data from other bright 47 Tuc sources (notably X7) and in ACIS-S spectra of the Crab Nebula (ObsID 2000). X10's spectrum is consistent with those seen from other polars, having a soft blackbody-like feature from the white dwarf surface in addition to a multitemperature accretion column (Ramsay et al. 2004). Detailed time-resolved analysis of this source, the first polar CV to be identified in a globular cluster, will be presented in $\mathrm{C}$. O. Heinke et al. (2005, in preparation). We note that both CVs X9 and $\mathrm{X} 10$ require plasma components with temperatures above $10 \mathrm{keV}$, showing that they too may follow the empirical relation between X-ray luminosity and $k T$ described above $(\S 7.1)$.

The CVs W33 and AKO 9 (W36) are not well fitted by MEKAL or power-law spectra. Both also have hard colors (see $\S 8$ ), like the eclipsing absorbed CVs W15 and W8 (G01a). AKO 9, however, appeared quite soft in the 2000 data set. Spectral fits to $\mathrm{AKO} 9$ require at least two plasma components $(k T=$ 0.6 and $10 \mathrm{keV})$, with the higher temperature component requiring additional absorption by a column of at least $3 \times 10^{22} \mathrm{H}$ $\mathrm{cm}^{-2}$. This is consistent with AKO 9's known eclipsing behavior (Edmonds et al. 1996; Knigge et al. 2003), which implies that we see the accretion impact zone on the white dwarf (Patterson \& Raymond 1985) through the edge of the accretion disk. The 2000 data show only one, unabsorbed component, best fitted at $0.3 \mathrm{keV}$; if the second, high-temperature component is present, it must be even more heavily absorbed (see Fig. 18). W33 also requires absorption by two different columns; a MEKAL model with temperature $1.8 \mathrm{keV}, 95 \%$ covered with $6 \times 10^{22} \mathrm{H}$ $\mathrm{cm}^{-2}$ (the remainder covered with $0.3 \times 10^{22} \mathrm{H} \mathrm{cm}^{-2}$ ), gives a good fit. The origin of the low-temperature components is unclear; perhaps they are scattered in an extended region, as observed in other CVs (Mukai et al. 2003; Pratt et al. 2004). For comparison with the relatively unusual spectra of $\mathrm{X} 9, \mathrm{X} 10$, and AKO 9, we also show the relatively simple spectrum of

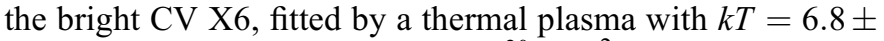
$0.8 \mathrm{keV}$ and $N_{\mathrm{H}}=(10.5 \pm 1.0) \times 10^{20} \mathrm{~cm}^{-2}$. Additional details on the spectra of all the 47 Tuc CVs will be presented in future papers.

\subsubsection{Additional $q L M X B s$ ?}

The three sources W37, W17, and X4 (W125) have unusual locations in our X-ray CMD (Fig. 9) and show poor fits (nhp $<5 \%$ ) to thermal plasma models (Table 7). Fitting them with two-component thermal plasmas gives an acceptable fit for $\mathrm{X} 4$, but not for the other two. W17 and X4 are also poorly fitted by blackbody, bremsstrahlung, and power-law models. These three sources are also the three brightest X-ray sources $\left(L_{\mathrm{X}} \sim\right.$ $4 \times 10^{31} \mathrm{ergs} \mathrm{s}^{-1}$ ) without known optical IDs in 47 Tuc. We 

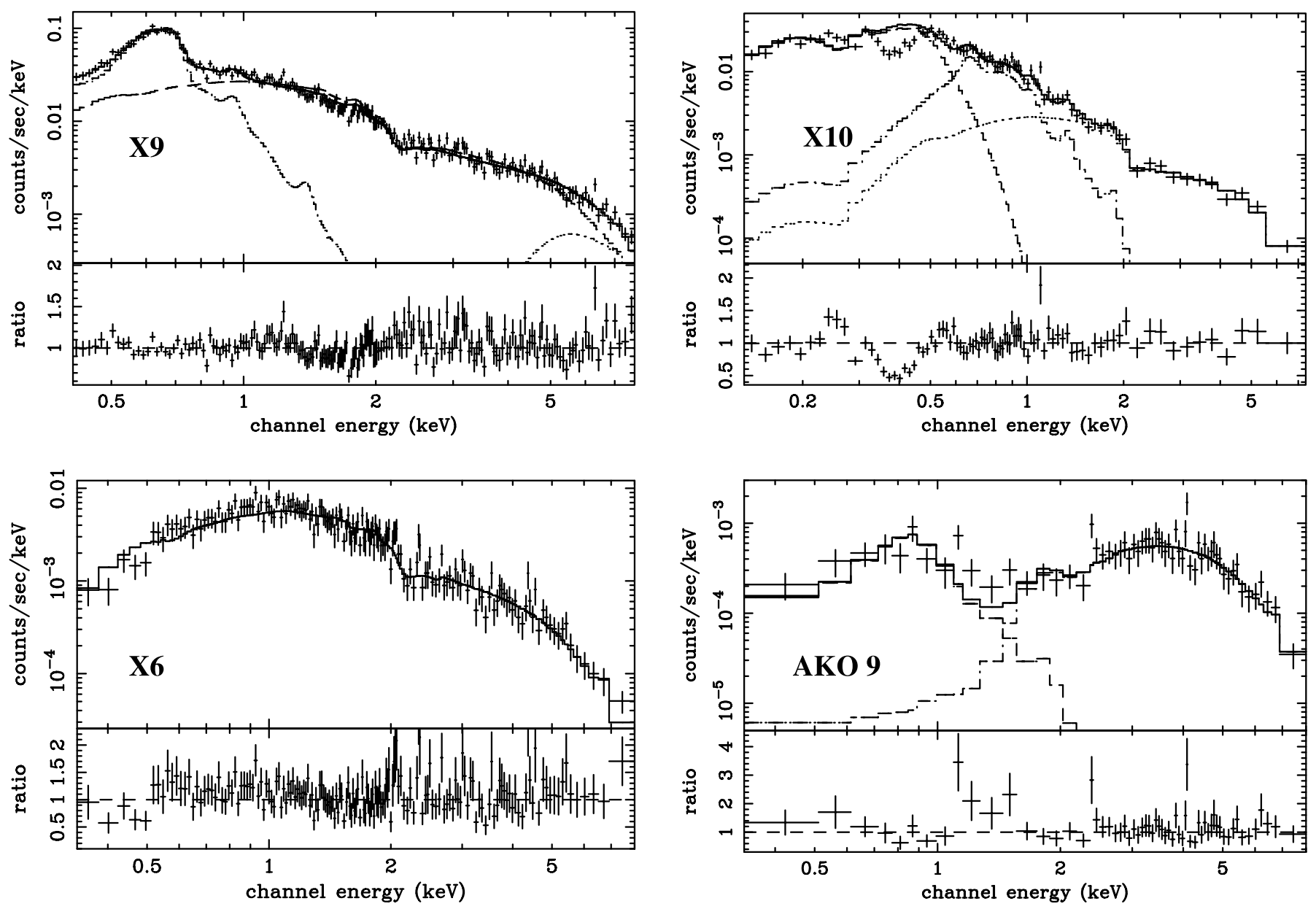

FIG. 18.-Energy spectra of four CVs in 47 Tuc, showing their diversity in observational appearance. Top left: X9 (W42), a bright CV that may be an intermediate polar, modeled with three MEKAL components at $0.25,>17$, and $>6 \mathrm{keV}$. The third component is absorbed behind a column of $9 \times 10^{23} \mathrm{H} \mathrm{cm}{ }^{-2}$. Top right: X10 (W27), a probable polar $\mathrm{CV}$, modeled with a $k T=53 \mathrm{eV}$ blackbody and two MEKAL plasmas at 0.39 and $>14 \mathrm{keV}$. The feature at $0.4 \mathrm{keV}$ is a known calibration residual. Bottom left: X6 (W56), a bright CV in 47 Tuc well fitted with a simple absorbed thermal plasma model, with enhanced $N_{\mathrm{H}}$ over the cluster value. Bottom right: AKO 9 (W36), an eclipsing CV with a subgiant secondary. We model the spectrum with two MEKAL components at 0.6 and $>5 \mathrm{keV}$ in the 2002 observations. The second component is absorbed behind a column of $5 \times 10^{22} \mathrm{H} \mathrm{cm}^{-2}$. In 2000, a single plasma component of temperature $0.3 \mathrm{keV}$ is seen; if the second component is present, it is obscured behind $>50 \times 10^{22} \mathrm{H} \mathrm{cm}^{-2}$ in the 2000 observations.

note that $\mathrm{W} 17$ is suggested as a possible qLMXB by Edmonds et al. (2003a). Quiescent LMXBs have been identified at X-ray luminosities as low as $10^{31} \mathrm{ergs} \mathrm{s}^{-1}$ and with relatively hard spectra (Campana et al. 2002; Tomsick et al. 2004). Fits of hydrogen atmosphere models plus power laws to W17 and X4 give implied surface radii $\left(12_{-4}^{+10}\right.$ and $7_{-1}^{+4} \mathrm{~km}$, respectively) consistent with 10-13 km, as expected for neutron stars (Lattimer $\&$ Prakash 2001), and power-law photon indices of 1.9, similar to those observed for qLMXBs in the field (Rutledge et al. 2002). W37's time-averaged spectrum is also well fitted by a hydrogen atmosphere plus power-law model, but the implied radius is only $1.7_{-0.3}^{+0.6} \mathrm{~km}$. However, W37 shows some of the most dramatic temporal and spectral variability in 47 Tuc, and time-resolved spectra show consistency with a $10 \mathrm{~km}$ radius for the thermal component. In a companion paper (Heinke et al. 2005 ) we show that W37's variability is due to varying $N_{\mathrm{H}}$ and eclipses and argue that $\mathrm{W} 17, \mathrm{X} 4$, and $\mathrm{W} 37$ are qLMXBs.

\section{DISCUSSION}

\subsection{MSPS}

For the first time, we have clearly detected in X-rays all MSPs in 47 Tuc with radio timing positions that are not located within $1^{\prime \prime}$ of another MSP. This allows us to measure the luminosity function for MSPs in 47 Tuc, describing them as a lognormal distribution with mean $\log L_{\mathrm{X}}=30.6$ and standard deviation $\log L_{\mathrm{X}}=0.28(0.5-2.5 \mathrm{keV})$.

We compare the $0.5-2.5$ and $0.5-6 \mathrm{keV} \mathrm{X}$-ray luminosities (from Table 2) of the individually detected MSPs to their radio pseudoluminosities (Camilo et al. 2000), to look for any correlation. For MSPs that do not have listed fluxes in Camilo et al. (2000), we use their upper limit estimate of $0.04 \mathrm{mJy} \mathrm{kpc}^{2}$. We show the radio pseudoluminosities and X-ray $0.5-6 \mathrm{keV}$ luminosities in Figure 19. Using a Spearman rank-order test (Press et al. 1992), we find statistically insignificant negative correlations (rank correlations $r_{s}=-0.05$ and -0.01 for $0.5-2.5$ and $0.5-6 \mathrm{keV}$ luminosities, respectively, with probabilities of chance occurrence of a correlation of this strength being $86 \%$ and $98 \%$ ). This analysis includes rough estimates of the pseudoluminosities of three MSPs (W, R, and T) that do not have measured flux densities listed in Camilo et al. (2000). Leaving out these three, we find statistically insignificant positive correlations (rank correlations $r_{s}=0.07$ and 0.14 , chance probabilities $82 \%$ and $69 \%$ ).

We conclude that we see no correlation of the radio pseudoluminosities from 47 Tuc MSPs with their X-ray luminosities, 


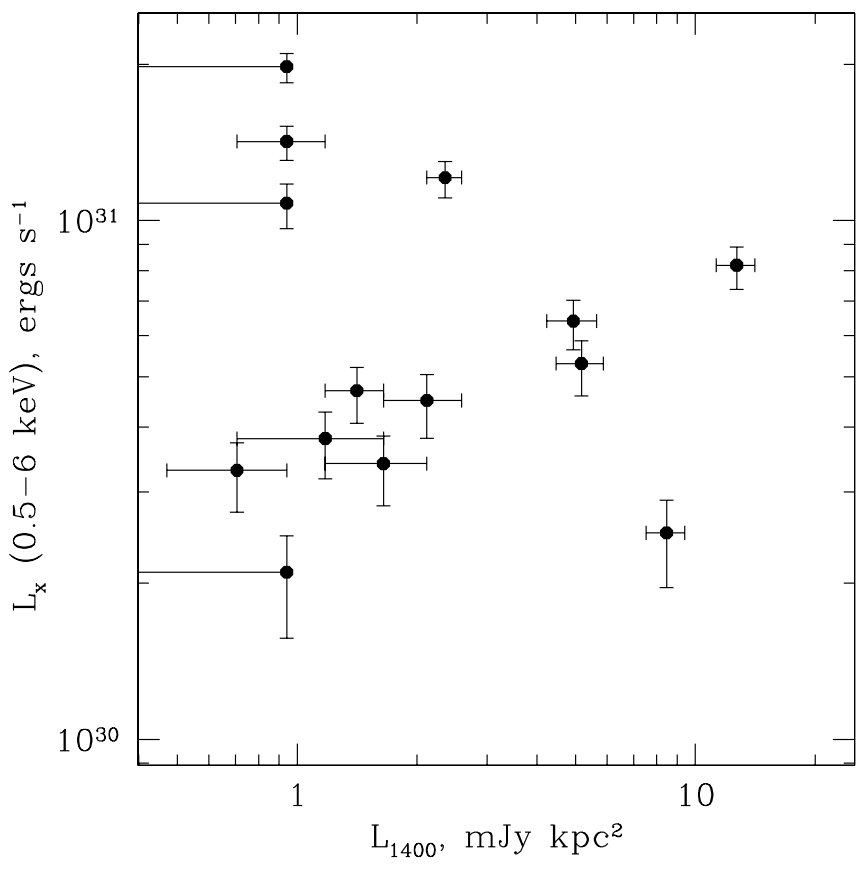

FIG. 19.-X-ray (0.5-6 keV) and radio (1400 MHz, pseudo-) luminosities for individually detected MSPs in 47 Tuc. Radio pseudoluminosities are from Camilo et al. (2000), with estimates for MSPs W, R, and T $\left(0.94 \mathrm{mJy} \mathrm{kpc}^{2}\right)$ taken as upper limits. No correlation is seen between X-ray and radio luminosities.

in agreement with Grindlay et al. (2002). This is not unexpected, since the X-ray emission from 47 Tuc MSPs is largely thermal radiation from the surface (Grindlay et al. 2002) while the radio emission originates in magnetospheric processes above the neutron star. This lack of any correlation between X-ray and radio signals indicates that additional MSPs in 47 Tuc will probably have X-ray luminosities similar to those MSPs that have already been identified. Some MSPs in 47 Tuc may have qualities that make them particularly difficult to detect in the radio, such as extremely short (less than $2 \mathrm{~ms}$ ) periods, or radio eclipses covering large parts of the orbit. Very short period MSPs might be expected to have relatively large spin-down luminosities and probably relatively high X-ray luminosities. Those MSPs in 47 Tuc that eclipse also tend to have relatively high X-ray luminosities ( $\mathrm{W}, \mathrm{O}$, and $\mathrm{J}$ are among the five X-ray brightest MSPs in 47 Tuc). It thus seems likely that MSPs with such qualities would be more, rather than less, X-ray luminous.

Among the 12 candidate MSPs listed by Edmonds et al. (2003b), W99 is identified as a cosmic ray in the 2000 data, not a real source. Four exhibit variability within the 2002 observations (W6, W96, W31, W91), and the last three of these show colors rather different from the majority of the known MSPs. W97, W95, and W115 appear much fainter in 2002 than the known MSPs $\left(L_{\mathrm{X}}<\frac{1}{2}\right.$ that of MSP 47 Tuc T, the faintest X-ray MSP known). This leaves W5, W28, W142, W34, and possibly W6 as excellent MSP candidates within the GO-8267 HST field and G01a source list. We identify another group of possible MSP candidates by their failure to be well fitted by VMEKAL models, together with their location in the colorcolor diagram. These additional MSP candidates are W10 (also a possible CV candidate), W65, W90, W40, W83, W6, W84, W253, W249, W303, W200, and W279. These 12 objects are likely to contain some MSPs but may also contain some other objects.

\section{2. $A B s$}

It has generally been believed (G01a) that ABs only appear as X-ray sources in globular clusters briefly, during flares, and then disappear. This survey indicates that ABs can maintain relatively high $\mathrm{X}$-ray luminosities over a period of weeks. One of the brighter ABs (W41, $L_{\mathrm{X}}>2 \times 10^{31} \mathrm{ergs} \mathrm{s}^{-1}$ ) was not detected as variable within our observations on any timescale. Although many ABs do show flaring behavior, their light curves are not easily distinguishable from those of CVs (see Fig. 6).

Thirty-one ABs have been newly identified as X-ray sources in 47 Tuc, and additional ABs will surely be identified using new and archival HST imaging. We note that of the eight classes of variables in 47 Tuc discussed by A01, we have detected 11 of $15 \mathrm{~W}$ UMa binaries, 5 of 6 red straggler variables, 8 of 10 eclipsing binaries, and 24 of 69 BY Draconis stars as X-ray sources. X-ray observations are apparently highly effective at identifying short-period binaries in globular clusters.

\subsection{Background Sources}

In $\S 4$ we showed that the distribution of sources with greater than 20 counts is consistent with a flat distribution beyond $100^{\prime \prime}$ out to our 2.'79 limiting survey radius. Thus, these sources are likely to be background, in that they are not a dynamically relaxed component of 47 Tuc. However, in $\S 6$ we showed that they are more numerous than the expectations for extragalactic background sources. Several explanations are possible, of which the most interesting is the possibility that some of these sources are associated with the halo of 47 Tuc. If they are halo sources, they are probably CVs and ABs formed by primordial binaries (Davies 1997; Gendre et al. 2003). Such a twocomponent distribution of interacting binaries has already been inferred for blue stragglers in M3 and 47 Tuc (Ferraro et al. 1997,2004 ). We note that only one X-ray source (W211, a faint AB) has been identified beyond $100^{\prime \prime}$ in 47 Tuc. A future study will examine the sources beyond the half-mass radius of 47 Tuc, to determine whether a substantial halo population exists.

Our sensitivity to faint X-ray sources drops off as we approach the crowded core of 47 Tuc. Thus, extrapolating the numbers of detected sources beyond $100^{\prime \prime}(55)$ to the area inside that limit will overestimate the number of background sources we have detected. A detailed analysis of our sensitivity limit through artificial-source simulations, required to understand the effects of crowding, is beyond the scope of this work. We estimate the total number of X-ray-detected background objects within the half-mass radius of 47 Tuc to be $\sim 70$.

\subsection{Unidentified Sources: Total Number of MSPS}

We have identified several differences between the MSPs in 47 Tuc and the other source classes. In this section we attempt to estimate the makeup of a subset of the unidentified sources by comparing their properties with those of the identified sources, with the goal of constraining the number of MSPs in 47 Tuc. We consider 120 sources with between 30 and 350 counts and sufficient spectral bins for spectral fitting, which include all individually identified MSPs (and no qLMXBs). We specify five classes: identified MSPs (16), ABs (27), CVs (7), likely background sources (those beyond $100^{\prime \prime} ; 14$ ), and the remaining unidentified sources (including CV, MSP, and AB candidates; 56). To constrain the total numbers of a source population, we require that the identified members were identified by a process that does not depend on X-ray characteristics and that unidentified 
members have similar X-ray characteristics (colors, luminosity, variability) to identified members. Given the measured X-ray luminosities of MSPs, the fact that all MSPs with a known position were detected in our data, and the lack of correlation between MSP X-ray luminosities and radio pseudoluminosities, we expect nearly all additional unidentified MSPs to fall among our studied subsample and to have similar properties to the detected MSPs. These criteria are not satisfied by CVs and ABs, so we cannot effectively constrain their populations by this method (see $\S 8.5)$.

Our method is based on the assumption that the unidentified sources are composed of a combination of sources similar to the identified sources,

$$
u=k a+l b+m c+n d
$$

with $u$ being the number of unidentified sources, $a, b, c$, and $d$ the numbers of MSPs, ABs, CVs, and background sources, and $k, l, m$, and $n$ ratios of unidentified to identified sources of each nature. We further assume that for each subset $i$ of sources identified by some property (such as variability), we can write

$$
u_{i} \approx k a_{i}+l b_{i}+m c_{i}+n d_{i}
$$

with $u_{i}$ being the number of unidentified sources sharing a property, $a_{i}$ being the number of known MSPs sharing this property, and $k a_{i}$ being the number of MSPs among the unknown sources sharing this property. Thus, we assume that the ratio of the number of known MSPs sharing a property to the number of unidentified MSPs sharing that property is the same (within errors determined by binomial statistics) as the ratio of the total number of known MSPs to the total number of unidentified MSPs.

We perform a least-squares fitting of the numbers of objects of each class to the total number of unidentified objects, within a number of groups. We define these groups by one of the following properties: the sources are variable, or nonvariable; the VMEKAL spectral fit is adequate, or it is poor (nhp $<5 \%$ ); or the X-ray colors are within one of three ranges (HR2 $<0.2$ and $\mathrm{HR} 3<-0.5$; HR2 $>0.2$ and HR3 $<-0.5$; HR3 $>-0.5$ ). Each of these groups is subdivided into two subgroups, including sources with 30-100 counts and those with 100350 counts, making a total of 14 groups. We define our merit function

$$
\chi^{2}=\sum_{i=1} \frac{\left(-u_{i}+k a_{i}+l b_{i}+m c_{i}+n d_{i}\right)^{2}}{\sigma_{u_{i}}^{2}+k^{2} \sigma_{a_{i}}^{2}+l^{2} \sigma_{b_{i}}^{2}+m^{2} \sigma_{c_{i}}^{2}+n^{2} \sigma_{d_{i}}^{2}},
$$

where $\sigma_{a_{i}}$ is the uncertainty on the random variable $a_{i}$, computed according to the binomial $1 \sigma$ limits of equations (21) and (26) of Gehrels (1986). We take the relevant rates to be $a_{i}$ and $a-a_{i}$ for computation of these binomial errors. We vary $n$ within the range $0.56 \pm 0.11$, based on the background number density from $\S 4$ and the geometric areas within and outside $100^{\prime \prime}$, to appropriately scale the numbers of background sources we should detect. We vary $k, l, m$, and $n$ to find the lowest value of $\chi^{2}$ and define our (enclosed $68 \%$ confidence) errors as $\Delta \chi^{2}=1$.

Forcing the coefficients $k, l$, and $m$ to be identical for the $30-100$ count bin and the 100-350 count bin, we derive $k=$ $0.20_{-0.2}^{+0.6}, l=1.75_{-0.35}^{+0.35}, m=0^{+0.8}$, or $3_{-3}^{+10}$ additional detected MSPs, $47_{-9}^{+9}$ additional ABs, and $0^{+6}$ additional $\mathrm{CVs}$ within this luminosity range. The limit on CVs is not a believable con- straint because we anticipate that some faint CVs may resemble ABs in their X-ray properties (see $\S 7$ ). Some MSPs may be confused with other sources as bright as themselves. This affects the known MSPs equally (two pairs are confused), and thus we multiply our MSP predictions by 1.125 to account for undetected sources. We arrive at $(16+3) \times 1.125=22_{-4}^{+7}$ total MSPs in 47 Tuc, with a $95 \%$ confidence single-sided upper limit of 40 MSPs.

This fit effectively incorporates some information about the luminosity functions, which are not well determined for $\mathrm{CVs}$ and $\mathrm{ABs}$, into the fitting. We relax the requirement that $k, l$, and $m$ be equal for both sources with more than 100 counts and those with less than 100 counts, by performing the fits separately for sources above and below 100 counts and combining the results. We find $30_{-12}^{+14}$ MSPs, $95 \%$ confidence upper limit of 54. Including all 132 sources between 30 and 350 counts and excluding information about the quality of spectral fits, we find $18_{-0}^{+15}$ MSPs, 95\% confidence upper limit 45, or $27_{-9}^{+16}$ MSPs and upper limit 57 if $k, l$, and $m$ are not held equal for both bins (thus ignoring both spectral fit and luminosity information). Considering these various estimates, we suggest 25 as the most likely number of MSPs in 47 Tuc and 60 as a conservative upper limit. A total of 22 MSPs are known through radio studies of 47 Tuc (four without known positions), providing an independent lower limit on the number of MSPs in 47 Tuc that is close to our predicted value.

MSPs that are continually enshrouded in ionized gas from their companion and thus invisible to radio searches (similar to 47 Tuc V and W; Camilo et al. 2000; Freire 2005), or in highly accelerated orbits, or with submillisecond spin periods will be included in this estimate if their X-ray properties are similar to those of the known MSPs. However, MSPs completely enshrouded in ionized gas may have hard X-ray spectra, caused by a shock from the pulsar wind interacting with the gas from the companion (as indicated for 47 Tuc W; Bogdanov et al. 2005). This would alter the X-ray properties of these enshrouded MSPs, so they would not be counted in our census. However, the X-ray luminosity from the wind shock must be much larger than the thermal MSP X-ray emission to dominate the X-ray spectrum, so such MSPs should be relatively bright $\left[L_{X}(0.5-\right.$ $6 \mathrm{keV}) \gtrsim 10^{31} \mathrm{ergs} \mathrm{s}^{-1}$, like 47 Tuc W]. There are only six unidentified sources within $100^{\prime \prime}$ of 47 Tuc with $L_{X}(0.5-6 \mathrm{keV})>$ $10^{31} \mathrm{ergs} \mathrm{s}^{-1}$, which limits the numbers of such enshrouded MSPs possible in 47 Tuc.

Because the X-ray beaming fraction is close to unity (S. Bogdanov et al. 2005, in preparation), our result constrains the radio beaming fraction to $\gtrsim 37 \%$ (in accord with the predictions of Lyne \& Manchester 1988). We note that our constraint on the total number of MSPs in 47 Tuc is in agreement with recent studies using optical identifications of X-ray sources (Edmonds et al. 2003b) and limits on the integrated radio flux from the cluster (McConnell et al. 2004), which argue that the total number of MSPs in 47 Tuc is probably $\lesssim 30$.

If we assume that MSPs are subject to the same scaling with close encounter frequency as LMXBs and qLMXBs (Verbunt \& Hut 1987; Johnston et al. 1992; Pooley et al. 2003; Heinke et al. 2003c), then our result predicts of order 700 MSPs in the Galactic globular cluster system. This comes from the fact that 47 Tuc contains $3.6 \%$ of the total close encounter rate of the Galactic globular cluster system (Heinke et al. 2003c). Predictions of substantially larger numbers of MSPs (e.g., 10,000 in Kulkarni et al. 1990) have been used to argue that the MSP birthrate is too high to be explained solely by their formation in LMXB systems. Our estimate helps alleviate the discrepancy 
between LMXB and MSP birthrates. We note, however, that another important factor in reconciling these birthrates may be the prediction that many MSPs were formed in the distant past through the evolution of intermediate-mass X-ray binaries (Podsiadlowski et al. 2002).

Edmonds et al. (2002a) showed that 47 Tuc W has probably exchanged the companion that originally spun it up to millisecond periods for another, main-sequence companion. Grindlay et al. (2002) argued that this was also a likely scenario for the eclipsing MSP J1740-5340 in NGC 6397. Freire (2005) suggested that all six observed eclipsing, low-mass $\left(M_{c} \sim 0.1-\right.$ $0.3 M_{\odot}$ ) binary pulsars, which are only observed in globular clusters, have undergone this interaction. (We follow Freire in using low-mass binary pulsars to mean those with companion masses $>0.1 M_{\odot}$, as opposed to very low mass binary pulsars with lower companion masses.) If this rate of exchanges is typical of neutron stars in general, Freire (2005) showed that this implied that about $7.5 \%$ of the neutron stars in MSPforming clusters may have been recycled into MSPs. This calculation is particularly applicable to 47 Tuc, which apparently has two eclipsing low-mass binary pulsars (Freire 2005; 47 Tuc $\mathrm{W}$ and probably $\mathrm{V}$ ) among 22 known radio MSPs. (It is also supported by recent theoretical work, such as Ivanova et al. [2005b], who produce recycling rates of 5\%-13\% in globular cluster models.) Dividing our estimate of 25 MSPs in 47 Tuc by 0.075 , we estimate that of order 330 neutron stars exist in 47 Tuc. This number carries large systematic uncertainties that we conservatively estimate as a factor of 4 . However, we believe that this is the first estimate driven purely by observations of the total number of neutron stars in a globular cluster. This estimate significantly alleviates the long-standing problem of neutron star retention in globular clusters (Pfahl et al. 2002a), especially when combined with our current understanding of neutron star kick distributions (Arzoumanian et al. 2002; Pfahl et al. 2002b).

\subsection{Unidentified Sources: CVs versus $A B s$}

Since the physical origin of the X-ray emission is similar for ABs and CVs (i.e., thermal emission from optically thin gas), it is difficult to clearly separate them. The X-ray properties of identified CVs also may differ from those of unidentified CVs, since the known CVs are X-ray selected.

An alternative approach to separating the CVs and ABs is to extrapolate from the identified objects in the GO-8267 HST field of view to the remaining sources. The GO-8267 data set is the most sensitive for detection of both $\mathrm{ABs}$ and CVs, owing to its extensive time series information, although several CVs have been identified in other HST data sets. A total of 153 X-ray sources (in the 2002 data) lie in the GO-8267 field of view, of which 57 are known ABs (26 identified in Edmonds et al. [2003a] and 31 identified here from A01 variables), 15 are CVs, 3 are qLMXBs (including the qLMXB candidate W17), and 12 are MSPs. Of order 10 may be background sources. In the rest of our field there are 147 sources, of which 60 are likely background sources. This allows us to directly extrapolate that at least $15 / 153 \times 87+15=24 \mathrm{CVs}$ and $57 / 153 \times 87+57=$ 89 ABs should be found in 47 Tuc. Since there still remain 66 unidentified sources in the GO-8267 field, these are lower limits. Since the ABs are less centrally concentrated than the other sources $(\S 4)$ and the GO-8267 field encloses the cluster core, the number of ABs must be even greater.

Upper limits may be set by assigning the remaining unidentified nonbackground X-ray sources (once the expected MSP contribution has been subtracted) to either ABs or CVs. In this way, we restrict the $\mathrm{X}$-ray-detected $\mathrm{AB}$ population to $\lesssim 178$ and the X-ray-detected CV population to $\lesssim 113$. This does not account for confusion, nor for objects not detected in the X-ray; unlike for MSPs, we have reason to believe that both ABs and CVs may exist at fainter luminosities than our survey reaches (Verbunt et al. 1997; Dempsey et al. 1997). Our nondetection of the candidate CVs detected in the far-UV by Knigge et al. (2002) also suggests that X-ray-faint CVs may lie below our detection limit. Further HST counterpart searches on both new and archival data will surely identify additional ABs and CVs among the detected X-ray sources. Only 17 of 77 real X-ray sources from the 2000 Chandra data set in the GO-8267 HST field of view remain unidentified $(22 \%)$, when the five $\mathrm{AB}$ candidates showing enhanced $N_{\mathrm{H}}$ (see $\S 7.1$ ), six MSPs with radio positions but no optical counterparts, and three qLMXBs are considered. We anticipate, therefore, that at least half the new unidentified sources in the GO-8267 field may be identified using new and archival HST data.

\section{CONCLUSIONS}

We have detected $300 \mathrm{X}$-ray sources within the half-mass radius of 47 Tuc in a deep $281 \mathrm{ks}$ Chandra ACIS-S observation taken in late 2002 , reaching down to $L_{\mathrm{X}}<8 \times 10^{29} \mathrm{ergs} \mathrm{s}^{-1}$. All 18 MSPs with known positions in 47 Tuc are detected, although two closely spaced pairs of MSPs are unresolved. All but 3 of 146 sources identified in the 72 ks Chandra ACIS-I observations in 2000 are detected in the new observations. We confidently identify $31 \mathrm{X}$-ray sources with ABs from the optical variable lists of A01, in addition to the $22 \mathrm{CVs}$ and $29 \mathrm{ABs}$ optically identified by Edmonds et al. (2003a). Seventy-eight X-ray sources show evidence of variability, at the $99 \%$ confidence level or higher, on timescales from hours to weeks within the 2002 data set. Nine additional sources show variability between the 2000 and 2002 observations.

Based on the radial distributions of the X-ray sources, roughly 70 sources are attributed to a background population that is not concentrated around the cluster center. The luminosity function for the observed background population implies an additional source population besides the expected extragalactic contribution. The radial profile of the sources associated with the cluster core allows an estimate of the mass ratio as $(1.63 \pm 0.11) M_{*}$, where $M_{*}$ is the average mass of the stars used to determine the core radius in Howell et al. (2000), and thus an inferred average system mass of $M=1.43 \pm 0.17 M_{\odot}$. This is consistent with our expectations for binary and/or heavy X-ray sources. The $\mathrm{AB}$ population is found to be significantly less massive, with $M=0.99 \pm 0.13 M_{\odot}$ implied. The MSP radial distribution implies an average system mass of $M=1.47 \pm$ $0.19 M_{\odot}$. Subtracting the known average mass of the binary counterparts gives an average neutron star mass of $1.39 \pm 0.19 M_{\odot}$, indicating that these MSPs have accreted very little mass during their recycling.

The X-ray colors and spectra of the X-ray sources are generally consistent with those expected from a thermal plasma model, in some cases requiring increased $N_{\mathrm{H}}$ over the known cluster value. The MSPs are generally not consistent with a thermal plasma model. Their colors suggest that their spectra lie between the predictions of blackbody and power-law models. The spectra of both the CVs and ABs tend to become harder as their X-ray luminosity increases. The bright CVs tend to show increased $N_{\mathrm{H}}$ over the cluster value. Some of these CVs are known to be eclipsing (and thus high inclination), but the prevalence of high $N_{\mathrm{H}}$ among bright CVs may suggest a magnetic DQ Her nature for the bright systems. The X-ray sources identified by Edmonds et al. (2003b) as possible ABs (on the 
basis of proximity to bright subgiant or giant stars that are saturated in the $H S T$ data) are unusual in that $5 / 6$ of them require high $N_{\mathrm{H}}$ values, indicating that the bright stars are indeed associated with the X-ray sources.

The two previously identified qLMXBs in 47 Tuc display the same spectral and variability characteristics as in the 2000 data set (Heinke et al. 2003b). We identify three additional likely qLMXBs (W37, W17, and X4=W125). These three objects stand out because of their soft colors and unusual spectra and require a spectral component that resembles a neutron star surface (Heinke et al. 2005). The bright CV known as X9 exhibits a strong $0.65 \mathrm{keV}$ emission line, likely $\mathrm{O}$ vIII. We are not aware of another spectrum of a $\mathrm{CV}$ showing such a strong flux from such a low temperature $(0.25 \mathrm{keV})$ component. The CV X10 shows strong flux below $0.5 \mathrm{keV}$ and sinusoidal modulations with a $16.8 \mathrm{ks}$ period, identifying it as a magnetic AM Her system (the first confirmed in a globular cluster). Using the numbers of detected CVs and ABs in the deeply studied GO-8267 HST field, we constrain the numbers of CVs and ABs among our detected 47 Tuc sources to $24-113 \mathrm{CVs}$ and 89-178 ABs. Additional CVs and ABs probably lie below our detection limit.

No correlation is found between the radio and X-ray luminosities of MSPs in 47 Tuc, motivating us to use the properties of detected MSPs to constrain the population of undetected MSPs, which we assume are similar. The difference in colors between MSPs and objects dominated by optically thin plasma radiation, along with variability and spectral fitting informa- tion, can be used to constrain the total number of MSPs in 47 Tuc to $22_{-4}^{+7}(95 \%$ confidence upper limit of $\sim 60)$, regardless of their radio beaming fraction. This allows us to estimate the total number of MSPs in the Galactic globular cluster system (assuming formation in the same manner as LMXBs) as $\sim 700$. If the eclipsing low-mass ( $\gtrsim 0.1 M_{\odot}$ companion) binary pulsar systems are good tracers for the likelihood of multiple recycling episodes for neutron stars, then we can estimate the total number of neutron stars in 47 Tuc as $\sim 300$.

Future papers will probe the spectra, variability, radial distributions, and optical properties of each of the classes of sources in 47 Tuc in greater detail. Our new simultaneous HST ACS data, along with archival HST data, will also enable many additional optical identifications.

We thank the Penn State team, particularly Pat Broos, for the development and support of the ACIS_EXTRACT software. We thank D. Lloyd for the use of his neutron star atmosphere models and M. Albrow for the results of his HST radial completeness calculations. We also thank R. Wijnands and R. di Stefano for useful discussions, R. Narayan for useful comments on the manuscript, and the referee for a careful and helpful report. This work has made substantial use of NASA's Astrophysics Data System and was supported in part by Chandra grant GO2-3059A. C. H. also acknowledges the support of the Lindheimer fund at Northwestern University.

\section{REFERENCES}

Albrow, M. D., Gilliland, R. L., Brown, T. M., Edmonds, P. D., Guhathakurta, P., \& Sarajedini, A. 2001, ApJ, 559, 1060 (A01)

Anderson, A. J. 1997, Ph.D. thesis, Univ. California, Berkeley

Arnaud, K. A. 1996, in ASP Conf. Ser. 101, Astronomical Data Analysis Software and Systems V, ed. G. H. Jacoby \& J. Barnes (San Francisco: ASP), 17 Arzoumanian, Z., Chernoff, D. F., \& Cordes, J. M. 2002, ApJ, 568, 289

Auriere, M., Koch-Miramond, L., \& Ortolani, S. 1989, A\&A, 214, 113

Backer, D. C. 1998, ApJ, 493, 873

Bailyn, C. D., Grindlay, J. E., \& Garcia, M. R. 1990, ApJ, 357, L35

Bailyn, C. D., \& Pinsonneault, M. H. 1995, ApJ, 439, 705

Bassa, C., et al. 2004, ApJ, 609, 755

Beloborodov, A. M. 2002, ApJ, 566, L85

Bergbusch, P. A., \& VandenBerg, D. A. 2001, ApJ, 556, 322

Bhattacharya, D., \& van den Heuvel, E. P. J. 1991, Phys. Rep., 203, 1

Bogdanov, S., Grindlay, J. E., \& van den Berg, M. 2005, ApJ, submitted

Brandt, W. N., et al. 2001, AJ, 122, 2810

Briley, M. M., Harbeck, D., Smith, G. H., \& Grebel, E. K. 2004, AJ, 127, 1588

Broos, P., Townsley, L., Getman, K., \& Bauer, F. 2002, ACIS Extract, An ACIS

Point Source Extraction Package, Pennsylvania State Univ., http://www.astro. psu.edu/xray/docs/TARA/ae_users_guide.html

Brown, E. F., Bildsten, L., \& Rutledge, R. E. 1998, ApJ, 504, L95

Camilo, F., Lorimer, D. R., Freire, P., Lyne, A. G., \& Manchester, R. N. 2000, ApJ, 535, 975

Campana, S., Colpi, M., Mereghetti, S., Stella, L., \& Tavani, M. 1998, A\&A Rev., 8, 279

Campana, S., Israel, G. L., Stella, L., Gastaldello, F., \& Mereghetti, S. 2004, ApJ, 601, 474

Campana, S., et al. 2002, ApJ, 575, L15

Cardelli, J. A., Clayton, G. C., \& Mathis, J. S. 1989, ApJ, 345, 245

Carney, B. W. 1996, PASP, 108, 900

Chandrasekhar, S., \& Münch, G. 1950, ApJ, 111, 142

Clark, G. W. 1975, ApJ, 199, L143

Cool, A. M., Grindlay, J. E., Cohn, H. N., Lugger, P. M., \& Slavin, S. D. 1995, ApJ, 439, 695

Cool, A. M., Haggard, D., \& Carlin, J. L. 2002, in ASP Conf. Ser. 265, Omega Centauri, A Unique Window into Astrophysics, ed. F. van Leeuwen, J. D. Hughes, \& G. Piotto (San Francisco: ASP), 277

Crawford, D. F., Jauncey, D. L., \& Murdoch, H. S. 1970, ApJ, 162, 405

Cropper, M., Ramsay, G., \& Wu, K. 1998, MNRAS, 293, 222

D’Amico, N., Possenti, A., Fici, L., Manchester, R. N., Lyne, A. G., Camilo, F., \& Sarkissian, J. 2002, ApJ, 570, L89
Davies, M. B. 1997, MNRAS, 288, 117

de Marchi, G., Paresce, F., Stratta, M. G., Gilliland, R. L., \& Bohlin, R. C. 1996, ApJ, 468, L51

Dempsey, R. C., Linsky, J. L., Fleming, T. A., \& Schmitt, J. H. M. M. 1993, ApJS, 86, 599

. 1997, ApJ, 478, 358

Edmonds, P. D., Gilliland, R. L., Camilo, F., Heinke, C. O., \& Grindlay, J. E. 2002a, ApJ, 579, 741

Edmonds, P. D., Gilliland, R. L., Guhathakurta, P., Petro, L. D., Saha, A., \& Shara, M. M. 1996, ApJ, 468, 241

Edmonds, P. D., Gilliland, R. L., Heinke, C. O., \& Grindlay, J. E. 2003a, ApJ, 596,1177

2003b, ApJ, 596, 1197

Edmonds, P. D., Gilliland, R. L., Heinke, C. O., Grindlay, J. E., \& Camilo, F. 2001, ApJ, 557, L57

Edmonds, P. D., Grindlay, J. E., Cool, A., Cohn, H., Lugger, P., \& Bailyn, C. 1999, ApJ, 516, 250

Edmonds, P. D., Heinke, C. O., Grindlay, J. E., \& Gilliland, R. L. 2002b, ApJ, $564, \mathrm{~L} 17$

Eracleous, M., Halpern, J., \& Patterson, J. 1991, ApJ, 382, 290

Favata, F., \& Schmitt, J. H. M. M. 1999, A\&A, 350, 900

Feigelson, E. D., et al. 2002, ApJ, 574, 258

Ferraro, F. R., Beccari, G., Rood, R. T., Bellazzini, M., Sills, A., \& Sabbi, E. 2004, ApJ, 603, 127

Ferraro, F. R., D’Amico, N., Possenti, A., Mignani, R. P., \& Paltrinieri, B. 2001, ApJ, 561, 337

Ferraro, F. R., et al. 1997, A\&A, 324, 915

Fregeau, J. M., Gürkan, M. A., Joshi, K. J., \& Rasio, F. A. 2003, ApJ, 593, 772

Freire, P. C. 2005, in ASP Conf. Ser. 328, Binary Radio Pulsars, ed. F. Rasio \& I. Stairs, in press (astro-ph/0404105)

Freire, P. C., Camilo, F., Kramer, M., Lorimer, D. R., Lyne, A. G., Manchester, R. N., \& D'Amico, N. 2003, MNRAS, 340, 1359

Freire, P. C., Camilo, F., Lorimer, D. R., Lyne, A. G., Manchester, R. N., \& D’Amico, N. 2001, MNRAS, 326, 901

Geffert, M., Auriere, M., \& Koch-Miramond, L. 1997, A\&A, 327, 137

Gehrels, N. 1986, ApJ, 303, 336

Gendre, B., Barret, D., \& Webb, N. A. 2003, A\&A, 400, 521

Giacconi, R., et al. 2001, ApJ, 551, 624

Gilliland, R. L., et al. 2000, ApJ, 545, L47

Gratton, R. G., et al. 2003, A\&A, 408, 529 
Grindlay, J. E., Camilo, F., Heinke, C. O., Edmonds, P. D., Cohn, H., \& Lugger, P. 2002, ApJ, 581, 470

Grindlay, J. E., Cool, A. M., Callanan, P. J., Bailyn, C. D., Cohn, H. N., \& Lugger, P. M. 1995, ApJ, 455, L47

Grindlay, J. E., Heinke, C., Edmonds, P. D., \& Murray, S. S. 2001a, Science, 292, 2290 (G01a)

Grindlay, J. E., Heinke, C. O., Edmonds, P. D., Murray, S. S., \& Cool, A. M. 2001b, ApJ, 563, L53

Grindlay, J. E., Hertz, P., Steiner, J. E., Murray, S. S., \& Lightman, A. P. 1984, ApJ, 282, L13

Harris, W. E. 1996, AJ, 112, 1487

Hasinger, G., Johnston, H. M., \& Verbunt, F. 1994, A\&A, 288, 466

Heinke, C. O., Grindlay, J. E., \& Edmonds, P. D. 2005, ApJ, 622, 556

Heinke, C. O., Grindlay, J. E., Edmonds, P. D., Lloyd, D. A., Murray, S. S., Cohn, H. N., \& Lugger, P. M. 2003a, ApJ, 598, 516

Heinke, C. O., Grindlay, J. E., Lloyd, D. A., \& Edmonds, P. D. 2003b, ApJ, 588,452

Heinke, C. O., Grindlay, J. E., Lugger, P. M., Cohn, H. N., Edmonds, P. D., Lloyd, D. A., \& Cool, A. M. 2003c, ApJ, 598, 501

Hertz, P., \& Grindlay, J. E. 1983, ApJ, 275, 105

Howell, J. H., Guhathakurta, P., \& Gilliland, R. L. 2000, PASP, 112, 1200

Hut, P., et al. 1992, PASP, 104, 981

Ivanova, N., Belczynski, K., Fregeau, J. M., \& Rasio, F. A. 2005a, MNRAS, 358,572

Ivanova, N., Fregeau, J. M., \& Rasio, F. A. 2005b, in ASP Conf. Ser. 328, Binary Radio Pulsars, ed. F. A. Rasio \& I. H. Stairs (San Francisco: ASP), in press (astro-ph/0405382)

Johnston, H. M., Kulkarni, S. R., \& Phinney, E. S. 1992, in X-Ray Binaries and the Formation of Binary and Millisecond Radio Pulsars, ed. E. P. J. Van Den Heuvel \& S. A. Rappaport (Dordrecht: Kluwer), 349

Kaluzny, J., Kubiak, M., Szymanski, M., Udalski, A., Krzeminski, W., Mateo, M., \& Stanek, K. Z. 1998, A\&AS, 128, 19

Kim, D.-W., et al. 2004, ApJ, 600, 59

King, I. R. 1966, AJ, 71, 64

Knigge, C., Zurek, D. R., Shara, M. M., \& Long, K. S. 2002, ApJ, 579, 752

Knigge, C., Zurek, D. R., Shara, M. M., Long, K. S., \& Gilliland, R. L. 2003, ApJ, 599, 1320

Kulkarni, S. R., Narayan, R., \& Romani, R. W. 1990, ApJ, 356, 174

Lattimer, J. M., \& Prakash, M. 2001, ApJ, 550, 426

Lewin, W. H. G., \& Joss, P. C. 1983, in Accretion-Driven Stellar X-Ray Sources, ed. W. H. G. Lewin \& E. P. J. van den Heuvel (Cambridge: Cambridge Univ. Press), 41

Lyne, A. G., \& Manchester, R. N. 1988, MNRAS, 234, 477

Maggio, A., Pallavicini, R., Reale, F., \& Tagliaferri, G. 2000, A\&A, 356, 627

Manchester, R. N., Lyne, A. G., Robinson, C., Bailes, M., \& D’Amico, N. 1991, Nature, 352, 219

McConnell, D., Deshpande, A. A., Connors, T., \& Ables, J. G. 2004, MNRAS, 348, 1409

Metchev, S. A., \& Grindlay, J. E. 2002, MNRAS, 335, 73

Mewe, R. 1991, A\&A Rev., 3, 127

Mukai, K., Still, M., \& Ringwald, F. A. 2003, ApJ, 594, 428

Muno, M. P., et al. 2003, ApJ, 589, 225

. 2004, ApJ, 613, 1179
Murdoch, H. S., Crawford, D. F., \& Jauncey, D. L. 1973, ApJ, 183, 1

Norton, A. J., \& Watson, M. G. 1989, MNRAS, 237, 853

Paresce, F., \& de Marchi, G. 1994, ApJ, 427, L33

Paresce, F., de Marchi, G., \& Ferraro, F. R. 1992, Nature, 360, 46

Patterson, J., \& Raymond, J. C. 1985, ApJ, 292, 535

Pechenick, K. R., Ftaclas, C., \& Cohen, J. M. 1983, ApJ, 274, 846

Pfahl, E., Rappaport, S., \& Podsiadlowski, P. 2002a, ApJ, 573, 283

Pfahl, E., Rappaport, S., Podsiadlowski, P., \& Spruit, H. 2002b, ApJ, 574, 364

Phinney, E. S., \& Sigurdsson, S. 1991, Nature, 349, 220

Podsiadlowski, P., Rappaport, S., \& Pfahl, E. D. 2002, ApJ, 565, 1107

Pooley, D., et al. 2002a, ApJ, 569, 405 2002b, ApJ, 573, 184 2003, ApJ, 591, L131

Pratt, G. W., Mukai, K., Hassall, B. J. M., Naylor, T., \& Wood, J. H. 2004, MNRAS, 348, L49

Predehl, P., \& Schmitt, J. H. M. M. 1995, A\&A, 293, 889

Press, W. H., Teukolsky, S. A., Vetterling, W. T., \& Flannery, B. P. 1992, Numerical Recipes in FORTRAN: The Art of Scientific Computing (2nd ed.; Cambridge: Cambridge Univ. Press)

Pryor, C., \& Meylan, G. 1993, in ASP Conf. Ser. 50, Structure and Dynamics of Globular Clusters, ed. S. G. Djorgovski \& G. Meylan (San Francisco: ASP), 357

Ramsay, G., Cropper, M., Mason, K. O., Córdova, F. A., \& Priedhorsky, W. 2004, MNRAS, 347, 95

Rasio, F. A., Pfahl, E. D., \& Rappaport, S. 2000, ApJ, 532, L47

Rutledge, R. E., Bildsten, L., Brown, E. F., Pavlov, G. G., \& Zavlin, V. E. 2002, ApJ, 577, 346

Saito, Y., Kawai, N., Kamae, T., Shibata, S., Dotani, T., \& Kulkarni, S. R. 1997, ApJ, 477, L37

Salaris, M., \& Weiss, A. 1998, A\&A, 335, 943

Sanders, J. S., Fabian, A. C., Allen, S. W., \& Schmidt, R. W. 2004, MNRAS, 349,952

Shara, M. M., Bergeron, L. E., Gilliland, R. L., Saha, A., \& Petro, L. 1996, ApJ, 471, 804

Sidoli, L., Parmar, A. N., Oosterbroek, T., Stella, L., Verbunt, F., Masetti, N., \& Dal Fiume, D. 2001, A\&A, 368, 451

Thorsett, S. E., \& Chakrabarty, D. 1999, ApJ, 512, 288

Tomsick, J. A., Gelino, D. M., Halpern, J. P., \& Kaaret, P. 2004, ApJ, 610, 933

van Teeseling, A., Beuermann, K., \& Verbunt, F. 1996, A\&A, 315, 467

Verbunt, F., Bunk, W. H., Ritter, H., \& Pfeffermann, E. 1997, A\&A, 327, 602

Verbunt, F., Elson, R., \& van Paradijs, J. 1984, MNRAS, 210, 899

Verbunt, F., \& Hasinger, G. 1998, A\&A, 336, 895

Verbunt, F., \& Hut, P. 1987, in IAU Symp. 125, The Origin and Evolution of Neutron Stars, ed. D. J. Helfand \& J.-H. Huang (Dordrecht: Reidel), 187

Verbunt, F., \& Lewin, W. H. G. 2004, in Compact Stellar X-Ray Sources, ed. W. H. G. Lewin \& M. van der Klis (Cambridge: Cambridge Univ. Press), in press (astro-ph/0404136)

Verbunt, F., \& Meylan, G. 1988, A\&A, 203, 297

Weldrake, D. T., Sackett, P. D., Bridges, T. J., \& Freeman, K. C. 2004, AJ, 128, 736

Wu, K., Chanmugam, G., \& Shaviv, G. 1995, ApJ, 455, 260 